\title{
The adic, cuspidal, Hilbert eigenvarieties
}

Fabrizio Andreatta ${ }^{3}$, Adrian lovita ${ }^{1,2^{*}}$ and Vincent Pilloni ${ }^{4}$

${ }^{*}$ Correspondence:

adrian.iovita@concordia.ca

Concordia University, Montreal,

Canada

Full list of author information is

available at the end of the article
In memory of Robert Coleman.

\section{Abstract}

We construct adic, compactified eigenvarieties parameterizing adic overconvergent Hilbert modular eigenforms of finite slope by constructing integral families of modular sheaves on the relevant formal Shimura schemes.

\section{Contents}

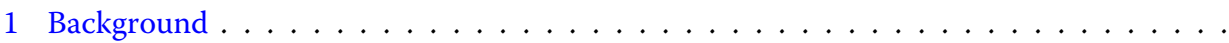

1.1 Modular sheaves associated with $G^{*} \ldots \ldots \ldots \ldots \ldots \ldots$

1.2 The modular sheaves associated with $G \ldots \ldots \ldots \ldots \ldots$

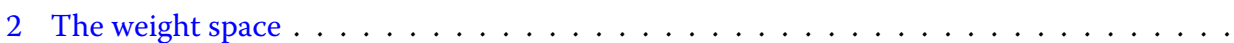

2.1 The Iwasawa algebra $\ldots \ldots \ldots \ldots \ldots \ldots \ldots$

2.2 A blowup of the formal weight space . . . . . . . . . . . . .

2.3 The adic weight space $\ldots \ldots \ldots \ldots \ldots \ldots \ldots$

2.4 Properties of the universal character . . . . . . . . . . . . . .

2.4 .1 Congruence properties . . . . . . . . . . . . . .

2.4 .2 A key lemma . . . . . . . . . . . . . . . . .

2.4.3 Analyticity of the universal character . . . . . . . . . . . .

3 Hilbert modular varieties and the Igusa tower . . . . . . . . . . . . . . .

3.1 Hilbert modular varieties . . . . . . . . . . . . . . . .

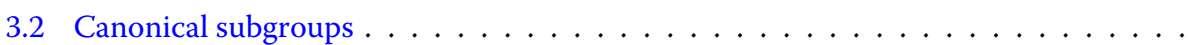

3.3 The partial Igusa tower $\ldots \ldots \ldots \ldots \ldots \ldots \ldots$

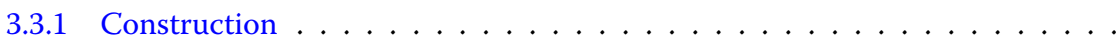

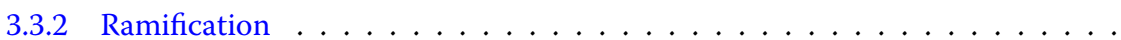

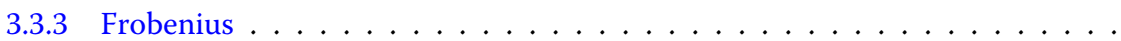

3.4 The basic constructions . . . . . . . . . . . . . . .

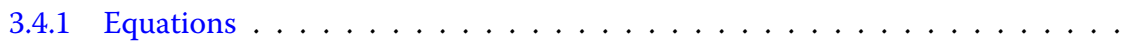

4 Overconvergent modular sheaves in characteristic $0 \ldots \ldots \ldots \ldots$

4.1 A modified integral structure on $\omega_{G} \ldots \ldots \ldots \ldots \ldots$

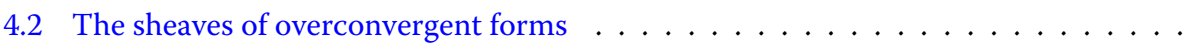

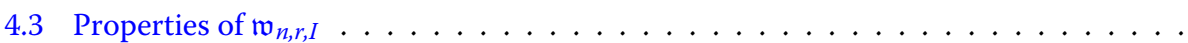

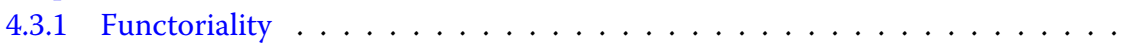

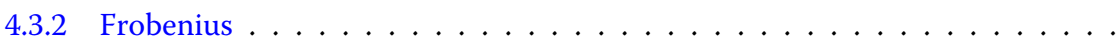

5 Perfect overconvergent modular forms

5.1 The anti-canonical tower

5.2 Tate traces

5.2 .1 An explicit description of diagram $(1)$ in Sect $5.2 \ldots \ldots \ldots \ldots \ldots$

(c) 2016 The Author(s). This article is distributed under the terms of the Creative Commons Attribution 4.0 International License (http://creativecommons.org/licenses/by/4.0/), which permits unrestricted use, distribution, and reproduction in any medium, provided you give appropriate credit to the original author(s) and the source, provide a link to the Creative Commons license, and indicate if changes were made. 


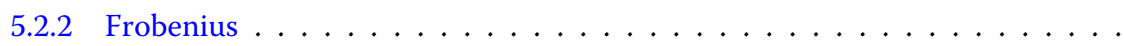

5.2 .3 The unramified case . . . . . . . . . . . . . . . . .

5.2 .4 The general case . . . . . . . . . . . . . . . .

5.3 The sheaf of perfect, overconvergent Hilbert modular forms . . . . . . . . . .

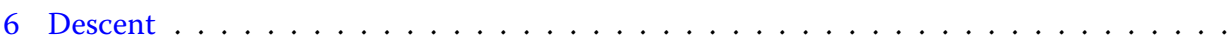

6.1 Comparison with the sheaf $\mathfrak{w}_{I} \ldots \ldots \ldots \ldots \ldots \ldots$

6.2 Descent along the anti-canonical tower $\ldots \ldots \ldots \ldots \ldots$

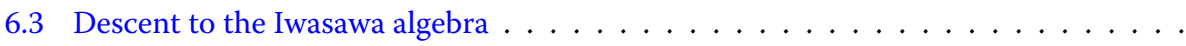

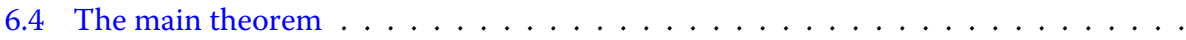

7 Overconvergent forms in characteristic $p \ldots \ldots \ldots \ldots \ldots$

7.1 The characteristic $p$ Igusa tower $\ldots \ldots \ldots \ldots \ldots \ldots$

7.2 Formal schemes attached to the Hilbert modular variety in characteristic $p \ldots \ldots$

7.3 Convergent Hilbert modular forms in characteristic $p \ldots \ldots \ldots$. . . . . . .

7.4 Overconvergent Hilbert modular forms in characteristic $p \ldots \ldots \ldots \ldots$

7.5 Comparison with the sheaf $\mathfrak{w}_{[1, \infty]} \ldots \ldots \ldots \ldots \ldots$

7.6 Analytic overconvergent Hilbert modular forms in characteristic $p \ldots \ldots$. . . .

8 Overconvergent arithmetic Hilbert modular forms . . . . . . . . . . . . . . .

8.1 The Shimura variety $\bar{M}\left(\mu_{N}, \mathfrak{c}\right)_{G} \ldots \ldots \ldots \ldots \ldots \ldots \ldots$

8.2 Descending the sheaf $\mathfrak{w}^{\kappa^{\text {un }}}$

8.3 The cohomology of the sheaf $\omega^{\kappa_{G}^{\text {un }}}(-D) \ldots \ldots \ldots \ldots \ldots \ldots \ldots \ldots \ldots \ldots \ldots \ldots \ldots \ldots$

8.4 Koecher principle and $q$-expansions . . . . . . . . . . . . . . . .

8.5 Hecke operators . . . . . . . . . . . . . . . . . .

8.6 The adic eigenvariety for arithmetic Hilbert modular forms . . . . . . . . . . . . .

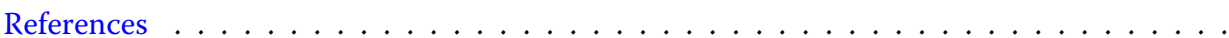

\section{Background}

During the 1990s, Robert Coleman constructed finite slope families of overconvergent, elliptic modular forms parametrized by rigid analytic subspaces of the weight space in [6, 7]. This was an extension of Hida's construction of ordinary families in the mid-1980s in [10]. One striking difference was that while Hida's theory was completely integral and worked over formal schemes, Coleman's theory was $\mathbb{Q}_{p}$-rigid analytic. Nevertheless, Coleman observed that the characteristic series of the $U_{p}$-operator acting on finite slope $p$-adic families of overconvergent modular form had coefficients in the Iwasawa algebra (i.e., they were integral) and conjectured that there should exist an integral or positive characteristic theory of overconvergent modular forms. Following Coleman's intuition, we obtained such a theory for elliptic modular forms in [3]. The present paper is an extension of [3] to the case of Hilbert modular forms.

More precisely, in the present paper we accomplish the following. Let us first fix a totally real number field $F$ of degree $g$ over $\mathbb{Q}$. Then let us recall (see, e.g., [2] or Sect. 8 of the present paper) that there are two relevant algebraic groups attached to $F$, denoted by $G:=\operatorname{Res}_{F / \mathbb{Q}} \mathbf{G L}_{2}$ and $G^{*}:=G \times \operatorname{Res}_{F / \mathbb{Q}} \mathbb{G}_{m} \mathbb{G}_{m}$.

From the point of view of automorphic forms, it is useful to work with modular forms on $G$, but the Shimura variety associated with $G$ is not a moduli space of abelian varieties. Instead, the Shimura variety associated with $G^{*}$ is a moduli space of abelian varieties, and so we first construct our modular sheaves for modular forms on $G^{*}$, as in [2], and then we descend these sheaves to the relevant varieties associated with $G$. 


\subsection{Modular sheaves associated with $G^{*}$}

This construction is accomplished in Sects. 1-7, where we work with toroidal compactifications of the moduli spaces of abelian schemes with $\mathcal{O}_{F}$-multiplication and we have a semi-abelian scheme which extends the universal abelian scheme.

To fix ideas, let $p$ denote a positive prime integer, $\mathbb{T}$ the torus $\operatorname{Res}_{F / \mathbb{Q}} \mathbb{G}_{m}$ and let $\Lambda_{F}:=$ $\mathbb{Z}_{p} \llbracket \mathbb{T}\left(\mathbb{Z}_{p}\right) \rrbracket$ be the associated Iwasawa algebra. We denote by $\mathcal{W}_{F}$ the analytic adic space (called the weight space for modular forms on $G^{*}$ ) associated with the formal scheme $\mathfrak{W}_{F}:=\operatorname{Spf} \Lambda_{F}$ and $\kappa^{\text {un }}: \mathbb{T}\left(\mathbb{Z}_{p}\right) \longrightarrow \Lambda_{F}^{\times}$the universal (weight) character. In particular, we have a natural decomposition of the adic weight space $\mathcal{W}_{F}=\mathcal{W}_{F}^{\text {rig }} \cup \mathcal{W}_{F, \infty}$, where $\mathcal{W}_{F}^{\text {rig }}$ is the adic space associated with the rigid analytic generic fiber of Spf $\Lambda_{F}$ (so this is the "old, $p$-adic weight space") and

$$
\mathcal{W}_{F, \infty}=\left\{\left.x \in \mathcal{W}_{F}|| p\right|_{x}=0\right\},
$$

sometimes called the "boundary of the weight space" and consisting in points with values in characteristic $p$-rings.

Let now $N \geq 4$ be an integer relatively prime to $p$ and let $\overline{\mathfrak{M}}\left(\mu_{N}, \mathfrak{c}\right)$ be the formal scheme associated with a projective toroidal compactification of the Shimura variety for $G^{*}$ of level $\left(\mu_{N}, \mathfrak{c}\right)$. Here $\mathfrak{c}$ is a fractional ideal of $F$ (see Sect. 3 for more details).

Our main result is the construction of an integral family of sheaves of overconvergent modular forms, parametrized by the formal spectrum of the Iwasawa algebra $\Lambda_{F}$. This overconvergent family extends the family of $p$-adic modular forms defined by Katz [14] and used by Hida in [11]. More precisely let us denote by $\mathfrak{W}_{F}^{0}=\operatorname{Spf} \Lambda_{F}^{0}$ the free" component of $\mathfrak{W}_{F}$, where $\Lambda_{F}^{0}$ is a complete regular local ring of dimension $g+1$ with maximal ideal $\mathfrak{m}$ and let $\mathfrak{Z}:=\overline{\mathfrak{M}}\left(\mu_{N}, \mathfrak{c}\right) \times \mathfrak{W}_{F}^{0}$. We consider, for each $r \geq 0$ the formal scheme $\mathfrak{Z}_{r}$, which should be thought of as a "formal neighborhood of the ordinary locus in $\mathfrak{Z}^{\prime}$ and which is defined as the formal scheme which represents the functor associated with every m-adically complete $\Lambda_{F}^{0}$-algebra $R$ the set of equivalence classes of tuples $\left(h, \eta_{p}, \eta_{1}, \eta_{2}, \ldots, \eta_{g}\right)$, where $h: \operatorname{Spf} R \longrightarrow \overline{\mathfrak{M}}\left(\mu_{N}, \mathfrak{c}\right)$ is a morphism of formal schemes and $\eta_{p}, \eta_{i} \in \mathrm{H}^{0}\left(\operatorname{Spf} R, h^{*}\left(\operatorname{det} \omega_{A}^{(1-p) p^{r+1}}\right)\right), i=1, \ldots, g$ satisfying

$$
\mathrm{Ha}^{p^{r+1}} \eta_{p}=p \bmod p^{2}, \mathrm{Ha}^{p^{r+1}} \eta_{1}=T_{1} \bmod p^{2}, \ldots, \mathrm{Ha}^{p^{r+1}} \eta_{g}=T_{g} \bmod p^{2} .
$$

See Sect. 6.3 for the definition of the equivalence relation between such tuples. Here $A$ is the universal semi-abelian scheme over $\overline{\mathfrak{M}}\left(\mu_{N}, \mathfrak{c}\right)$, denoted $G$ in the main body of the article, and $T_{1}, T_{2}, \ldots, T_{g}$ are chosen elements of $\mathfrak{m}$, which together with $p$ generate it (see Sect. 2.1 for more details). Let $\mathfrak{M}_{r}$ be the base change, as formal schemes, of $\mathfrak{Z}_{r}$ to $\mathfrak{W}_{F}$. We construct, for each $r \geq 0$, a coherent sheaf $\mathfrak{w}_{r}^{\kappa^{\text {un }}}$ on $\mathfrak{M}_{r}$. Let $\mathcal{M}_{r}$ denote the adic analytic space associated with $\mathfrak{M}_{r}$ and $\omega_{r}^{\text {un }}$ the associated analytic coherent sheaf. Then $\omega_{r}^{\kappa^{\text {un }}}$ is invertible and it satisfies the following properties.

1. The restriction of $\omega_{r}^{\text {un }}$ to the rigid analytic space $\mathcal{M}_{r} \times \operatorname{Spa}\left(\mathbb{Z}_{p}, \mathbb{Z}_{p}\right) \operatorname{Spa}\left(\mathbb{Q}_{p}, \mathbb{Z}_{p}\right)$ is the sheaf defined in [2, Definition 3.6].

2. For all classical weights $k \cdot \chi: \mathbb{T}\left(\mathbb{Z}_{p}\right) \longrightarrow \mathcal{O}_{\mathbb{C}_{p}}^{*}$, where $k$ is an algebraic weight and $\chi$ a finite order character, the specialization of $\omega_{r}^{\text {un }}$ to $k \cdot \chi$ is the restriction to $\mathcal{M}_{r}$ of the sheaf $\omega_{A}^{k}(\chi)$ of classical modular forms of weight $k$ and nebentypus $\chi$.

3. The family of sheaves $\left\{\omega_{r}^{\text {un }}\right\}_{r \geq 0}$ is Frobenius compatible.

See Sect. 6.4. 


\subsection{The modular sheaves associated with $G$}

This construction is done in Sect. 8. Let $\mathfrak{W}_{F}^{G}, \kappa_{G}^{\text {un }}$ denote the formal weight space and respectively the universal character associated with the Iwasawa algebra $\Lambda_{F}^{G}$ for the group $G$ and let $\mathfrak{W}_{F}^{G} \longrightarrow \mathfrak{W}_{F}$ be the natural morphism of formal schemes. Let now $\overline{\mathfrak{M}}\left(\mu_{N}, \mathfrak{c}\right)_{G}$ denote the formal Shimura variety for the group $G$ (i.e., the formal completion along the special fiber of a projective, toroidal compactification of the Shimura variety for $G$ ). The toroidal compactifications for the Shimura varieties for $G^{*}$ and $G$ can be chosen in such a way that we have a natural morphism of formal schemes $\alpha: \overline{\mathfrak{M}}\left(\mu_{N}, \mathfrak{c}\right) \longrightarrow \overline{\mathfrak{M}}\left(\mu_{N}, \mathfrak{c}\right)_{G}$. Moreover, if $\Delta$ denotes the quotient of the group of totally real units of $\mathcal{O}_{F}$ by the square of the units congruent to 1 modulo $N$, this finite group acts naturally on $\overline{\mathfrak{M}}\left(\mu_{N}, \mathfrak{c}\right)$ by multiplication on the polarizations, such that:

1. The morphism $\alpha$ is finite, étale and Galois with Galois group $\Delta$. It follows that $\overline{\mathfrak{M}}\left(\mu_{N}, \mathfrak{c}\right)_{G} \cong\left(\overline{\mathfrak{M}}\left(\mu_{N}, \mathfrak{c}\right)\right) / \Delta$.

2. For every $r \geq 0$, we have a natural action of $\Delta$ on $\mathfrak{M}_{r} \times_{\mathfrak{W}_{F}} \mathfrak{W}_{F}^{G}$ lifting to an action on $\mathfrak{w}_{r}^{\kappa_{G}^{\text {un }}}$, which is the pullback of $\mathfrak{w}_{r}^{\kappa^{\text {un }}}$ to $\mathfrak{M}_{r} \times \mathfrak{W}_{F} \mathfrak{W}_{F}^{G}$. By finite étale descent, we obtain a coherent sheaf, still denoted $\mathfrak{w}_{r}^{\kappa_{G}^{\text {un }}}$, on $\mathfrak{M}_{r, G}:=\left(\mathfrak{M}_{r} \times \mathfrak{W}_{F} \mathfrak{W}_{F}^{G}\right) / \Delta$.

3. If we denote by $\mathcal{M}_{r, G}$ the analytic adic space associated with the formal scheme $\mathfrak{M}_{r, G}$ and by $\omega_{r}^{\kappa_{G}^{\mathrm{un}}}$ the associated coherent sheaf, then $\omega_{r}^{\kappa_{G}^{\mathrm{un}}}$ is invertible and the overconvergent modular forms for $G$ are overconvergent sections of specializations of this modular sheaf. As in [2], one can show by a cohomological argument that specialization is surjective on cuspidal forms.

The spectral theory of the operator $U_{p}$ on adic families of overconvergent modular forms allows us to construct an adic eigenvariety sitting over the analytic adic space associated with the Iwasawa algebra $\Lambda_{F}^{G}$. See Sect. 8.6.

Finally, this article generalizes and is crucially based on both [2,3]. In particular, for many arguments we refer to loc. cit. Let us point out what is really new here:

1. The boundaries of the weight spaces, for both $G$ and $G^{*}$, are analytic spaces of dimension $g-1$. Therefore, the boundary overconvergent Hilbert modular forms (i.e., the overconvergent Hilbert modular forms in characteristic $p$ ) are parameterized by positive dimensional analytic spaces if $g>1$, i.e., live in true analytic families.

2. In [3], the universal integral modular sheaf $\mathfrak{w}_{r}^{\text {un }}$ was a sheaf parameterized by the formal blowup of the formal scheme $\operatorname{Spf} \Lambda$ with respect to the ideal $\mathfrak{m}$. Therefore, the descent to the Iwasawa algebra in this paper improves [3].

3. If $p$ is ramified in $\mathcal{O}_{F}$, the descent of the perfect sheaves of overconvergent Hilbert modular forms to finite levels by the use of Tate traces involves new problems due to the non smoothness of the associated Hilbert modular varieties in characteristic $p$.

Remark 1.1 In [2] and also in this paper, we work with toroidal compactifications $\bar{M}\left(\mu_{N}, \mathfrak{c}\right)$ of the integral models of the Shimura varieties associated with $G^{*}$ defined by Deligne and Pappas [9], completing previous work of Rapoport [16]. These models are singular at primes dividing the discriminant of $F$. One could use one of the splitting models $\widetilde{\widetilde{M}}\left(\mu_{N}, \mathfrak{c}\right) \rightarrow \bar{M}\left(\mu_{N}, \mathfrak{c}\right)$ of $\bar{M}\left(\mu_{N}, \mathfrak{c}\right)$ introduced by Pappas and Rapoport [15]. Such models depend on some auxiliary choices, namely an ordering of the embeddings of $F$ in an algebraic closure, but they have the advantage of being smooth. The given map 
is an isomorphism over an open dense subscheme (the Rapoport locus, see Sect. 3); in particular, the two moduli spaces differ only at primes $p$ dividing the discriminant of $F$.

Recall that the complement of the Rapoport locus is characterized by the fact that the sheaf $\omega_{G}$ of invariant differentials of the versal semi-abelian scheme $G$ over $\bar{M}\left(\mu_{N}, \mathfrak{c}\right)$ is not locally free as $\mathcal{O}_{F} \otimes \mathcal{O}_{\bar{M}\left(\mu_{N}, \mathfrak{c}\right)}$-module. On the other hand on a splitting model, $\omega_{G}$ admits a filtration by invertible $\mathcal{O}_{\tilde{M}}\left(\mu_{N}, \mathfrak{c}\right)$-modules, stable for the action of $\mathcal{O}_{F}$. These invertible sheaves allow to define Hilbert modular forms of non-parallel weight over the whole of $\widetilde{\bar{M}}\left(\mu_{N}, \mathfrak{c}\right)$.

For our purposes, i.e., the construction of modular sheaves, it makes no difference which model we choose and we prefer to work with the minimal (and more canonical) one, the Deligne-Pappas model. The main reason is the fact that the key ingredient in the construction of the modular sheaves is the introduction of a different integral structure $\mathcal{F}$ of $\omega_{G}$ which is locally free as $\mathcal{O}_{F} \otimes \mathcal{O}_{\mathfrak{I} \mathfrak{G}_{h, l, I}}$-module, e.g., even on the complement of the Rapoport locus in the formal scheme $\mathfrak{I}_{n, r, I}$ (see [2], Proposition 4.1, or Sect. 4.1 of the present article).

Notations Let $F$ be a totally real number field. Denote by $g$ the degree $[F: \mathbb{Q}]$. Fix a prime $p$. Denote by $\mathfrak{P}_{1}, \ldots, \mathfrak{P}_{f}$ the prime ideals of $\mathcal{O}_{F}$ over $p$. For each $i$, let $f_{i}$ be the residual degree and $e_{i}$ the ramification index. Write $\mathfrak{p}=\mathfrak{P}_{1} \cdots \mathfrak{P}_{f}$ for the product of all the primes of $\mathcal{O}_{F}$ above $p$. Set $q=p$ if $p>2$ and $q=4$ if $p=2$.

\section{The weight space}

\subsection{The Iwasawa algebra}

Denote by $\mathbb{T}:=\operatorname{Res}_{\mathcal{O}_{F} / \mathbb{Z}} \mathbb{G}_{m}$ and by $\Lambda_{F}$ the completed group algebra $\mathbb{Z}_{p} \llbracket \mathbb{T}\left(\mathbb{Z}_{p}\right) \rrbracket$. We write

$$
\kappa^{\mathrm{un}}: \mathbb{T}\left(\mathbb{Z}_{p}\right) \rightarrow \Lambda_{F}^{*}
$$

for the universal character. Fix an isomorphism of topological groups

$$
\rho: H \times \mathbb{Z}_{p}^{g} \rightarrow \mathbb{T}\left(\mathbb{Z}_{p}\right)=\left(\mathcal{O}_{F} \otimes \mathbb{Z}_{p}\right)^{*}
$$

where $H$ is the torsion subgroup of $\mathbb{T}\left(\mathbb{Z}_{p}\right)$. Write $\Lambda_{F}^{0}$ for $\mathbb{Z}_{p} \llbracket \mathbb{Z}_{p}^{g} \rrbracket \cong \mathbb{Z}_{p} \llbracket T_{1}, \ldots, T_{g} \rrbracket$ where $1+T_{i}=\epsilon_{i}$, the $i$ th vector basis of $\mathbb{Z}_{p}^{g}$. It is a complete, regular, local ring with maximal ideal $\mathfrak{m}$. Furthermore, $\Lambda_{F} \cong \Lambda_{F}^{0}[H]$ is a finite flat $\Lambda_{F}^{0}$-algebra. Actually, there is also a canonical projection map $\Lambda_{F} \rightarrow \Lambda_{F}^{0}$ obtained by sending all $h \in H$ to 1 . We let $\kappa: \mathbb{T}\left(\mathbb{Z}_{p}\right) \rightarrow\left(\Lambda_{F}^{0}\right)^{\star}$ be the composition of $\kappa^{u n}$ and the above projection. We let $\chi: H \rightarrow \Lambda_{F}^{*}$ be the composition of the inclusion $H \hookrightarrow \mathbb{T}\left(\mathbb{Z}_{p}\right)$ and the universal character.

We denote by $\mathfrak{W}_{F}$ resp. $\mathfrak{W}_{F}^{0}$ the $\mathfrak{m}$-adic formal scheme defined by $\Lambda_{F}$ resp. $\Lambda_{F}^{0}$. Then we have a natural map $\mathfrak{W}_{F} \rightarrow \mathfrak{W}_{F}^{0}$ which is finite and flat.

Remark 2.1 In [2], the weight space has been defined over the ring of integers of a finite extension $K$ of $\mathbb{Q}_{p}$ splitting $F$. The reason is that the classical weights are defined over $K$. Here we prefer to work over $\mathbb{Z}_{p}$. As a consequence, it will turn out that the characteristic series of the $U_{p}$ operator will have coefficients in the Iwasawa algebra $\Lambda_{F}^{G}$ defined in Theorem 8.8, with no need to extend scalars.

\subsection{A blowup of the formal weight space}

Consider the blow-up Spec $\Lambda_{F}$ of Spec $\Lambda_{F}$ with respect to the ideal $\mathfrak{m}$ and let $\mathfrak{t}: \widetilde{\mathfrak{W}}_{F} \rightarrow \mathfrak{W}_{F}$ be the associated $\mathfrak{m}$-adic formal scheme. 
We describe in more detail the formal scheme $\widetilde{\mathfrak{W}}_{F}$. Notice that by the universal property of the blowup, the ideal sheaf $\mathfrak{I}:=\mathfrak{t}^{-1}(\mathfrak{m}) \subset \mathcal{O}_{\widetilde{\mathfrak{W}}_{F}}$ is invertible. For every element $\alpha \in \mathfrak{m}$, we denote by $\mathfrak{W}_{\alpha}=\mathfrak{D}_{+}(\alpha)=\operatorname{Spf}\left(B_{\alpha}\right) \subset \widetilde{\mathfrak{W}}_{F}$ the open affine formal subscheme where $\mathfrak{I}$ is generated by $\alpha\left(\mathfrak{W}_{\alpha}\right.$ is empty unless $\left.\alpha \in \mathfrak{m} \backslash \mathfrak{m}^{2}\right)$. In particular, the $\mathfrak{m}$-adic topology on $B_{\alpha}$ coincides with the $\alpha$-adic topology.

One has variants $\widetilde{\mathfrak{W}}_{F}^{0} \rightarrow \mathfrak{W}_{F}^{0}$ of the spaces introduced above and associated with the sub-algebra $\Lambda_{F}^{0}$ of $\Lambda_{F}$. We also have a natural finite and flat morphism $\widetilde{\mathfrak{W}}_{F} \rightarrow \widetilde{\mathfrak{W}}_{F}^{0}$. For every element $\alpha \in \mathfrak{m}$, we write $\mathfrak{W}_{\alpha}^{0}=\operatorname{Spf}\left(B_{\alpha}^{0}\right) \subset \widetilde{\mathfrak{W}}_{F}^{0}$ for the open affine formal subscheme defined by $\alpha$.

\subsection{The adic weight space}

Let $\widetilde{\mathcal{W}}_{F}$ be the analytic adic space associated with $\widetilde{\mathfrak{W}}_{F}$. For all open Spf $A$ of $\widetilde{\mathfrak{W}}_{F}$, the associated open of $\widetilde{\mathcal{W}}_{F}$ is the open subset of analytic points $\operatorname{Spa}(A, A)^{a n}$ of $\operatorname{Spa}(A, A)$. For every element $\alpha \in \mathfrak{m}$, let $\mathcal{W}_{\alpha}$ be the open subset of $\widetilde{\mathcal{W}}_{F}$ consisting of the analytic points of the adic space associated with $\mathfrak{W}_{\alpha}$. Then $\mathcal{W}_{\alpha}$ is affinoid equal to $\operatorname{Spa}\left(B_{\alpha}\left[\alpha^{-1}\right], B_{\alpha}\right)=$ $\operatorname{Spa}\left(B_{\alpha}, B_{\alpha}\right)^{a n}$.

Choosing generators $\left(p, T_{1}, \ldots, T_{g}\right)$ of $\mathfrak{m}$ then $\widetilde{\mathcal{W}}_{F}$ is covered by the affinoids

$$
\mathcal{W}_{p}, \mathcal{W}_{T_{1}}, \ldots, \mathcal{W}_{T_{g}}
$$

We let $\mathcal{W}_{F}$ be the analytic adic space associated with $\mathfrak{W}_{F}$. Namely, $\mathcal{W}_{F}$ consists of the analytic points $\operatorname{Spa}\left(\Lambda_{F}, \Lambda_{F}\right)^{a n} \subset \operatorname{Spa}\left(\Lambda_{F}, \Lambda_{F}\right)$. We denoteby $t: \widetilde{\mathcal{W}}_{F} \rightarrow \mathcal{W}_{F}$ the morphism of analytic adic spaces associated with $\mathfrak{t}: \widetilde{\mathfrak{W}}_{F} \rightarrow \mathfrak{W}_{F}$.

Lemma 2.2 The morphism $t: \widetilde{\mathcal{W}}_{F} \rightarrow \mathcal{W}_{F}$ is an isomorphism of adic spaces.

Proof For all $\alpha \in \mathfrak{m}$, the subset $\left\{x \in \mathcal{W}_{F}, 0 \neq|\alpha|_{x} \geq|\beta|_{x}, \forall \beta \in \mathfrak{m}\right\}$ of $\mathcal{W}_{F}$ equals $\mathcal{W}_{\alpha}$ by definition. Moreover, $\mathcal{W}_{F}$ is covered by the $\mathcal{W}_{\alpha}$. The conclusion follows.

Remark 2.3 Let us denote by $\mathcal{W}_{F}^{\text {Berk }}$ the subset of rank 1 points of $\mathcal{W}_{F}$. Then there is a map:

$$
\begin{aligned}
\Theta: \mathcal{W}_{F}^{\text {Berk }} & \rightarrow \mathbb{P}^{g}(\mathbb{R}) \\
x & \mapsto\left(|p|_{x},\left|T_{1}\right|_{x}, \ldots,\left|T_{g}\right|_{x}\right)
\end{aligned}
$$

with image included in $\left[0,1^{g+1}\right.$. This map may be helpful in order to understand $\mathcal{W}_{F}$. Let us denote by $\left(x_{0}, \ldots, x_{g}\right)$ the coordinates on $\mathbb{P}^{g}(\mathbb{R})$. Then $\Theta^{-1}\left(\left\{x_{0} \neq 0\right\}\right)$ is the set of rank one points on the usual (adic) weight space over $\operatorname{Spa}\left(\mathbb{Q}_{p}, \mathbb{Z}_{p}\right)$ associated with $\Lambda_{F}$.

Let us denote by $\mathcal{W}_{F}^{0}$ the analytic adic space attached to $\mathfrak{W}_{F}^{0}$. For every element $\alpha \in \mathfrak{m}$, we denote by $\mathcal{W}_{\alpha}^{0}$ the analytic adic space associated with $\mathfrak{W}_{\alpha}^{0}$.

Finally, let us remark that the classical weights are points of the subspace of $\mathcal{W}_{F}$ where $p$ is invertible.

\subsection{Properties of the universal character}

\subsubsection{Congruence properties}

First of all, we need to elaborate on the identification $\rho: H \times \mathbb{Z}_{p}^{g} \simeq\left(\mathcal{O}_{F} \otimes \mathbb{Z}_{p}\right)^{*}$ of the previous section.

Lemma 2.4 (1) The group $H$ can be realized as a quotient of $\left(\mathcal{O}_{F} \otimes \mathbb{Z}_{p}\right)^{*} /\left(1+q \mathcal{O}_{F} \otimes \mathbb{Z}_{p}\right)$. Its prime to $p$ part is isomorphic to $\left(\mathcal{O}_{F} \otimes \mathbb{Z}_{p}\right)^{*} /\left(1+\mathfrak{p} \mathcal{O}_{F} \otimes \mathbb{Z}_{p}\right)$.

(2) Given $\left(a_{1}, \ldots, a_{g}\right) \in \mathbb{Z}_{p}^{g}$, we have $\kappa\left(\rho\left(a_{1}, \ldots, a_{g}\right)\right)=\prod_{i=1}^{g}\left(1+T_{i}\right)^{a_{i}} \in\left(\Lambda_{F}^{0}\right)^{*}$. 
Proof (1) The group $H$ is finite and its prime to $p$ part maps isomorphically onto $\left(\mathcal{O}_{F} \otimes\right.$ $\left.\mathbb{Z}_{p}\right)^{*} /\left(1+\mathfrak{p} \mathcal{O}_{F} \otimes \mathbb{Z}_{p}\right)$ via $\rho$. Denote by $L$ the quotient $\left(\mathcal{O}_{F} \otimes \mathbb{Z}_{p}\right)^{*} / H$. The subgroup $1+q \mathcal{O}_{F} \otimes \mathbb{Z}_{p}$ of $\left(\mathcal{O}_{F} \otimes \mathbb{Z}_{p}\right)^{*}$ is isomorphic to $q\left(\mathcal{O}_{F} \otimes \mathbb{Z}_{p}\right)$, and hence to $\mathbb{Z}_{p}^{g}$, via the logarithm. In particular, it injects into $L$ via the quotient map and the subgroup $H$ of $\left(\mathcal{O}_{F} \otimes \mathbb{Z}_{p}\right)^{*}$ injects into $\left(\mathcal{O}_{F} \otimes \mathbb{Z}_{p}\right)^{*} /\left(1+q \mathcal{O}_{F} \otimes \mathbb{Z}_{p}\right)$. This proves the first claim.

(2) The standard basis elements $\epsilon_{1}, \ldots, \epsilon_{g}$ of $\mathbb{Z}_{p}^{g}$ map to $1+T_{1}, \ldots, 1+T_{g}$ in $\Lambda_{F}^{0}$.

We define the following ideals in $\Lambda_{F}^{0}$ :

- $\mathfrak{m}_{n}=\left(\alpha^{p^{n-1}}, p \alpha^{p^{n-2}}, \ldots, p^{n-1} \alpha, \alpha \in\left(T_{1}, \ldots, T_{g}\right)\right)$ if $n \geq 1$.

- $\mathfrak{m}_{0}=\mathfrak{m}_{1}=\left(T_{1}, \ldots, T_{g}\right)$.

Lemma 2.5 For every $n \in \mathbb{Z}_{\geq 1}$ we have that $\kappa\left(\rho\left(p^{n-1} \mathbb{Z}_{p}^{g}\right)\right)-1 \subset \mathfrak{m}_{n}$. In particular, we have for all $n \in \mathbb{Z}_{\geq 1}$

$$
\kappa\left(1+q p^{n-1} \mathcal{O}_{F} \otimes \mathbb{Z}_{p}\right)-1 \subset \mathfrak{m}_{n}
$$

Moreover, $\kappa\left(\mathbb{T}\left(\mathbb{Z}_{p}\right)\right)-1 \subset \mathfrak{m}_{0}$.

Proof Note that $\kappa\left(\rho\left(p^{n-1} a_{1}, \ldots, p^{n-1} a_{g}\right)\right)=\prod_{i=1}^{g}\left(1+T_{i}\right)^{p^{n-1} a_{i}}$. One computes that $\left(1+T_{i}\right)^{p^{n-1}}-1$ is contained in the ideal $\left(T_{i}^{p^{n-1}}, p T_{i}^{p^{n-2}}, \ldots, p^{n-1} T_{i}\right)$; see [3, Lemme 2.3].

Notice that $\kappa$ is trivial on $H$ so that it factors via $\rho\left(\mathbb{Z}_{p}^{g}\right) \cong\left(\mathcal{O}_{F} \otimes \mathbb{Z}_{p}\right)^{*} / H$. Furthermore, $\left(1+q p^{n-1} \mathcal{O}_{F} \otimes \mathbb{Z}_{p}\right)=\left(1+q \mathcal{O}_{F} \otimes \mathbb{Z}_{p}\right)^{p^{n-1}}$ (using the logarithm). In particular, $(1+$ $\left.q p^{n-1} \mathcal{O}_{F} \otimes \mathbb{Z}_{p}\right)$ is contained in $\rho\left(p^{n-1} \mathcal{O}_{F} \otimes \mathbb{Z}_{p}\right)$ via the identification above. The second claim follows.

\subsubsection{A key lemma}

We introduce a formalism inspired by Sen's theory that will be repeatedly used in the paper. Let $n \in \mathbb{Z}_{\geq 1}$ and $A_{0} \rightarrow A_{1} \cdots \rightarrow A_{n}$ be a tower of $\Lambda_{F}^{0}$-algebras which are domains. We assume that the group $\left(\mathcal{O}_{F} / p^{n} \mathcal{O}_{F}\right)^{\star}$ acts on $A_{n}$ by automorphisms of $\Lambda_{F}^{0}$-algebras and that $A_{s}$ is the subring of $A_{n}$ fixed by the kernel $H_{s}$ of the map $\left(\mathcal{O}_{F} / p^{n} \mathcal{O}_{F}\right)^{*} \rightarrow\left(\mathcal{O}_{F} / p^{s} \mathcal{O}_{F}\right)^{*}$.

Let $h \in A_{0}$ and let $p_{0}=0 \leq p_{1} \leq \cdots \leq p_{n}$ be a sequence of integers. Let $c_{n} \in h^{-p_{n}} A_{n}$ be an element. Set $c_{s}=\sum_{\sigma \in H_{s}} \sigma \cdot c_{n}$. We assume that:

- $c_{s} \in h^{-p_{s}} A_{s}$ for all $s \geq 0$,

- $c_{0}=1$.

Set $b_{s}=\sum_{\sigma \in\left(\mathcal{O}_{F} / p^{s} \mathcal{O}_{F}\right)^{*}} \kappa(\tilde{\sigma}) \sigma\left(c_{s}\right) \in h^{-p_{s}} A_{s}$ for $s \geq 1$ and $b_{0}=1$. Here $\tilde{\sigma} \in \mathbb{T}\left(\mathbb{Z}_{p}\right)$ is a lift of $\sigma$ so that $b_{s}$ depends on $c_{s}$ and on the choices of lifts.

Lemma 2.6 1. Another system of choices of lifts $\tilde{\sigma}$ for the $\sigma$ 's would give an element $b_{s}^{\prime}$ and we have

- $b_{s}^{\prime}-b_{s} \in h^{-p_{s}} \mathfrak{m}_{s} A_{s}$ if $s \geq 1, p \geq 3$,

- $b_{s}^{\prime}-b_{s} \in h^{-p_{s}} \mathfrak{m}_{s-1} A_{s}$ if $s \geq 2, p=2$,

- $b_{1}^{\prime}-b_{1} \in h^{-p_{1}} \mathfrak{m}_{0} A_{1}$ if $p=2$.

2. We have the following congruence relations:

- $b_{s}-b_{s-1} \in h^{-p_{s}} \mathfrak{m}_{s-1} A_{s}$ if $s \geq 1, p \geq 3$,

- $b_{s}-b_{s-1} \in h^{-p_{s}} \mathfrak{m}_{s-2} A_{s}$ if $s \geq 2, p \geq 3$,

- $b_{1}-b_{0} \in h^{-p_{1}} \mathfrak{m}_{0} A_{0}$ if $p=2$. 
Proof The first point follows from Lemma 2.5. To prove the second point, assume that $s \geq 1$ and notice that

$$
\begin{aligned}
b_{s} & =\sum_{\tau \in\left(\mathcal{O}_{F} / p^{s-1} \mathcal{O}_{F}\right)^{\star}} \kappa(\tilde{\tau}) \tau\left(\sum_{\sigma \in 1+p^{s-1} \mathcal{O}_{F} / p^{s} \mathcal{O}_{F}}(\kappa(\tilde{\sigma})-1) \sigma\left(c_{s}\right)+c_{s-1}\right) \\
& =\sum_{\tau \in\left(\mathcal{O}_{F} / p^{s-1} \mathcal{O}_{F}\right)^{\star}} \kappa(\tilde{\tau}) \tilde{\tau}\left(\sum_{\sigma \in 1+p^{s-1} \mathcal{O}_{F} / p^{s} \mathcal{O}_{F}}(\kappa(\tilde{\sigma})-1) \sigma\left(c_{s}\right)\right)+b_{s-1}
\end{aligned}
$$

One concludes by applying Lemma 2.5 and also using the first point.

\subsubsection{Analyticity of the universal character}

We now study the analytic properties of the universal character. The degree of analyticity depends on the $p$-adic valuation of $T_{1}, \ldots, T_{g}$. This motivates the following definition. For $\frac{r}{s} \in \mathbb{Q}_{\geq 1}$, we define the following rational open subsets of $\mathcal{W}_{F}^{0}$ :

- $\mathcal{W}_{F, \leq \frac{r}{s}}^{0}=\left\{x \in \mathcal{W}_{F}^{0},\left|\alpha^{r}\right|_{x} \leq\left|p^{s}\right|_{x} \neq 0, \forall \alpha \in \mathfrak{m}\right\}$,

- $\mathcal{W}_{F, \geq \frac{r}{s}}^{0}=\left\{x \in \mathcal{W}_{F}^{0}, \exists \alpha \in \mathfrak{m},\left|p^{s}\right|_{x} \leq\left|\alpha^{r}\right|_{x} \neq 0\right\}$.

Set $\mathcal{W}_{F, \leq \infty}^{0}:=\mathcal{W}_{F}^{0}$. If $I=[a, b]$ is a closed interval with $a, b \in \mathbb{Q}_{\geq 1} \cup\{\infty\}$, define $\mathcal{W}_{F, I}^{0}=\mathcal{W}_{F, \leq b}^{0} \cap \mathcal{W}_{F, \geq a}^{0}$. For all $\alpha \in \mathfrak{m}$, we let $\mathcal{W}_{\alpha, I}^{0}=\mathcal{W}_{F, I}^{0} \cap \mathcal{W}_{\alpha}^{0}$.

Remark 2.7 If $x \in \mathcal{W}_{\alpha}^{0}$ is a rank one point, then $\alpha$ is a pseudo-uniformizer of the residue field $k(x)$. Let us denote by $v_{\alpha}: k(x) \rightarrow \mathbb{R} \cup\{\infty\}$ the valuation on $k(x)$ normalized by $v_{\alpha}(\alpha)=1$. Notice that the norm $p^{-v_{\alpha}(\cdot)}$ represents the equivalence class of $|\cdot|_{x}$. Then $x \in \mathcal{W}_{\alpha, I}^{0}$ if and only if $v_{\alpha}(p) \in I$.

We now construct formal models. Take an element $\alpha \in \mathfrak{m}$. We define $B_{\alpha, I}^{0}=$ $\mathrm{H}^{0}\left(\mathcal{W}_{\alpha, \mathrm{I}}, \mathcal{O}_{\mathcal{W}_{\alpha, \mathrm{I}}}^{+}\right)$

Set $\mathfrak{W}_{\alpha, I}^{0}=\operatorname{Spf} B_{\alpha, I}^{0}$. The analytic fiber of $\mathfrak{W}_{\alpha, I}^{0}$ is $\mathcal{W}_{\alpha, I}^{0}$. For various $\alpha$ 's, the $\mathfrak{W}_{\alpha, I}^{0}$ glue to a formal scheme $\tilde{\mathfrak{W}}_{F, I}^{0}$ with analytic fiber $\mathcal{W}_{F, I}^{0}$. Remark that $\widetilde{\mathfrak{W}}_{F,[1, \infty]}^{0}=\widetilde{\mathfrak{W}}_{F}^{0}$.

If $I \subset\left[0, \infty\left[\right.\right.$, then $\widetilde{\mathfrak{W}}_{F, I}^{0}$ is a $p$-adic formal scheme (the $\mathfrak{m}$-adic topology is the $p$-adic one). In the lemma below, $\mathbb{G}_{m}, \mathbb{G}_{a}$ are considered as functors on the category of $p$-adic formal schemes equipped with a structural morphism to $\mathfrak{W}_{F}^{0}$. Let $\epsilon=1$ if $p \neq 2$ and $\epsilon=3$ if $p=2$. The group $\mathbb{T}\left(\mathbb{Z}_{p}\right) \cdot\left(1+p^{n+\epsilon} \mathcal{O}_{F} \otimes \mathbb{G}_{a}\right)$ is a subgroup of $\mathbb{G}_{m}$.

Proposition 2.8 Let $n \geq 0$ be an integer. Suppose that $I \subset\left[0, p^{n}\right]$. The character $\kappa$ extends to a pairing

$$
\widetilde{\mathfrak{W}}_{F, I}^{0} \times \mathbb{T}\left(\mathbb{Z}_{p}\right) \cdot\left(1+p^{n+\epsilon} \mathcal{O}_{F} \otimes \mathbb{G}_{a}\right) \longrightarrow \mathbb{G}_{m} .
$$

It restricts to a pairing

$$
\tilde{\mathfrak{W}}_{F, I}^{0} \times\left(1+p^{n+\epsilon+n^{\prime}} \mathcal{O}_{F} \otimes \mathbb{G}_{a}^{+}\right) \longrightarrow 1+q p^{n^{\prime}} \mathbb{G}_{a}
$$

for all $n^{\prime} \in \mathbb{Z}_{\geq 0}$.

Proof Easy and left to the reader.

\section{Hilbert modular varieties and the Igusa tower}

\subsection{Hilbert modular varieties}

We recall the definition of Hilbert modular varieties following $[9,16]$. 
Fix an integer $N \geq 4$ and a prime $p$ not dividing $N$. Let $\mathfrak{c}$ be a fractional ideal of $F$ and let $\mathfrak{c}^{+}$be the cone of totally positive elements. Denote by $\mathcal{D}_{F}$ the different ideal of $\mathcal{O}_{F}$. Let $M\left(\mu_{N}, \mathfrak{c}\right)$ be the Hilbert modular scheme over $\mathbb{Z}_{p}$ classifying triples $(A, \iota, \Psi, \lambda)$ consisting of: (1) abelian schemes $A \rightarrow S$ of relative dimension $g$ over $S$, (2) an embedding $\iota: \mathcal{O}_{F} \subset \operatorname{End}_{S}(A)$, (3) a closed immersion $\Psi: \mu_{N} \otimes \mathcal{D}_{F}^{-1} \rightarrow A$ compatible with $\mathcal{O}_{F^{-}}$ actions, and (4) if $P \subset \operatorname{Hom}_{\mathcal{O}_{F}}\left(A, A^{\vee}\right)$ is the sheaf for the étale topology on $S$ of symmetric $\mathcal{O}_{F}$-linear homomorphisms from $A$ to the dual abelian scheme $A^{\vee}$ and if $P^{+} \subset P$ is the subset of polarizations, then $\lambda$ is an isomorphism of étale sheaves $\lambda:\left(P, P^{+}\right) \cong\left(\mathfrak{c}, \mathfrak{c}^{+}\right)$, as invertible $\mathcal{O}_{F}$-modules with a notion of positivity. The triple is subject to the condition that the map $A \otimes \mathcal{O}_{F} \mathfrak{c} \rightarrow A^{\vee}$ is an isomorphism of abelian schemes (the so called DelignePappas condition).

We write $\bar{M}\left(\mu_{N}, \mathfrak{c}\right)$ and $\bar{M}^{*}\left(\mu_{N}, \mathfrak{c}\right)$ for a projective toroidal compactification, respectively the minimal or Satake compactification of $M\left(\mu_{N}, \mathfrak{c}\right)$ (see [16]). Let $\overline{\mathfrak{M}}\left(\mu_{N}, \mathfrak{c}\right)$ [resp. $\left.\overline{\mathfrak{M}}^{*}\left(\mu_{N}, \mathfrak{c}\right)\right]$ be the associated formal schemes. They are endowed with a semi-abelian scheme $G$ with $\mathcal{O}_{F}$-action.

There exist maximal open subscheme, respectively formal subscheme $\bar{M}^{\mathrm{R}}\left(\mu_{N}, \mathfrak{c}\right) \subset$ $\bar{M}\left(\mu_{N}, \mathfrak{c}\right)$, resp. $\overline{\mathfrak{M}}^{\mathrm{R}}\left(\mu_{N}, \mathfrak{c}\right) \subset \overline{\mathfrak{M}}\left(\mu_{N}, \mathfrak{c}\right)$ such that $\omega_{G}$, the conormal sheaf to the identity of $G$, is an invertible $\mathcal{O}_{\bar{M}^{\mathrm{R}}\left(\mu_{N}, \mathfrak{c}\right)} \otimes_{\mathbb{Z}} \mathcal{O}_{F}$-module, resp. $\mathcal{O}_{\overline{\mathfrak{M}}^{\mathrm{R}}\left(\mu_{N}, \mathfrak{c}\right)} \otimes_{\mathbb{Z}} \mathcal{O}_{F}$-module (the so called Rapoport condition). The complement is empty if $p$ does not divide the discriminant of $F$ and, in general, it is of codimension 2 in the characteristic $p$ special fiber of $\bar{M}\left(\mu_{N}, \mathfrak{c}\right)$; see [9].

We denote by $\mathrm{Ha} \in \mathrm{H}^{0}\left(M^{*}\left(\mu_{N}, \mathfrak{c}\right)_{\mathbb{F}_{p}}\right.$, det $\left.\omega_{G}^{p-1}\right)$ the Hasse invariant. We let Hdg $\subset$ $\mathcal{O}_{\overline{\mathfrak{M}}\left(\mu_{N}, \mathfrak{c}\right)}$ be the Hodge ideal defined by the Hasse invariant (see [3, $\mathbb{}$ A.1] for a precise definition: locally on $\overline{\mathfrak{M}}\left(\mu_{N}, \mathfrak{c}\right)$ it is the ideal generated by $p$ and a (any) lift of a local generator of Ha det $\omega_{G}^{1-p}$ ).

\subsection{Canonical subgroups}

Let $A_{0}$ be a $\mathbb{Z}_{p}$-algebra and $\alpha \in A_{0}$ a nonzero element. We assume that $A_{0}$ satisfies the following:

(*) $A_{0}$ is an integral domain, and it is the $\alpha$-adic completion of a $\mathbb{Z}_{p}$-algebra of finite type and $p \in \alpha A_{0}$.

Let $\bar{M}\left(\mu_{N}, \mathfrak{c}\right){ }$ Spec $\mathbb{Z}_{p}$ Spec $A_{0}$ be the base change of the toroidal compactification via Spec $A_{0} \rightarrow$ Spec $\mathbb{Z}_{p}$ and let $\mathfrak{Y}$ be the associated formal scheme over Spf $A_{0}$.

Definition 3.1 For every integer $r \in \mathbb{N}$ denote by $\mathfrak{Y}_{r} \rightarrow \mathfrak{Y}$ the formal scheme over $\mathfrak{Y}$ representing the functor which to any $\alpha$-adically complete $A_{0}$-algebra $R$ without $\alpha$-torsion associates the equivalence classes of pairs $\left(h: \operatorname{Spf} R \rightarrow \mathfrak{Y}, \eta \in \mathrm{H}^{0}\left(\operatorname{Spf} R, h^{*} \operatorname{det} \omega_{G}^{(1-p) p^{r+1}}\right)\right.$ such that

$$
\mathrm{Ha}^{p^{r+1}} \eta=\alpha \bmod p^{2}
$$

Two pairs $(h, \eta)$ et $\left(h^{\prime}, \eta^{\prime}\right)$ are declared equivalent if $h=h^{\prime}$ and $\eta=\eta^{\prime}\left(1+\frac{p^{2}}{\alpha} u\right)$ for some $u \in R$.

We also denote by $\mathfrak{Y}_{r}^{\mathrm{R}} \subset \mathfrak{Y}_{r}$ the open formal subscheme where the Rapoport condition holds (see Sect. 3). 
Proposition 3.2 Assume that $p \in \alpha^{p^{k}} A_{0}$. Then for every integer $1 \leq n \leq r+k$ one has a canonical subgroup scheme $H_{n}$ of $G\left[p^{n}\right]$ over $\mathfrak{Y}_{r}$ and $H_{n}$ modulo $p \mathrm{Hdg}^{-\frac{p^{n}}{p-1}}$ lifts the kernel of the $n$th power of Frobenius. Moreover, $H_{n}$ is finite flat and locally of rank $p^{\text {ng }}$, it is stable under the action of $\mathcal{O}_{F}$, and the Cartier dual $H_{n}^{D}$ is étale locally over $A_{0}\left[\alpha^{-1}\right]$ isomorphic to $\mathcal{O}_{F} / p^{n}$ (as $\mathcal{O}_{F}$-module).

Proof All claims follow from [3, Appendix A].

Proposition 3.3 For every $r \in \mathbb{Z}_{\geq 2}$, the isogeny given by dividing by the canonical subgroup $H_{1}$ of level 1 defines a finite morphism $\phi: \mathfrak{Y}_{r} \rightarrow \mathfrak{Y}_{r-1}$. The restriction to the Rapoport locus $\phi: \mathfrak{Y}_{r}^{\mathrm{R}} \rightarrow \mathfrak{Y}_{r-1}^{\mathrm{R}}$ is finite and flat of degree $p^{g}$.

Proof This is the content of [3, Cor. A.2] which is written for general $p$-divisible groups. The last claim follows as relative Frobenius is finite and it is flat over the (smooth) Rapoport locus.

\subsection{The partial Igusa tower}

\subsubsection{Construction}

We use the notations of the previous section. Let $A:=A_{0}\left[\alpha^{-1}\right]$ : It is a Tate ring in the sense of Huber [12] with ring of definition $A_{0}$. Let $A^{+} \subset A$ be the normalization of $A_{0}$ in $A$. The fact that $A_{0}$ is noetherian implies that $\operatorname{Spa}\left(A, A^{+}\right)$is an adic space; [13, Thm. 2.2]. We define

$$
\mathcal{Y}_{r}:=\mathfrak{Y}_{r}^{\mathrm{ad}} \times_{\operatorname{Spa}\left(A_{0}, A_{0}\right)} \operatorname{Spa}\left(A, A^{+}\right):
$$

here $\mathfrak{Y}_{r}^{\text {ad }}$, resp. $\mathrm{Spa}\left(A_{0}, A_{0}\right)$, is the adic space associated with the formal scheme $\mathfrak{Y}_{r}$, resp. Spf $A_{0}$, and the fiber product is taken in the category of adic spaces.

Assume that $p \in \alpha^{p^{k}} A_{0}$ and let $r \in \mathbb{N}$ and $n \in \mathbb{N}$ be an integer such that $1 \leq n \leq r+k$. It follows from Proposition 3.2 that $H_{n}^{D}$ over $\mathcal{Y}_{r}$ is étale locally isomorphic to $\mathcal{O}_{F} / p^{n} \mathcal{O}_{F}$. We let $\mathcal{I} \mathcal{G}_{n, r} \rightarrow \mathcal{Y}_{r}$ be the Galois cover for the group $\left(\mathcal{O}_{F} / p^{n} \mathcal{O}_{F}\right)^{*}$ classifying the isomorphisms $\mathcal{O}_{F} / p^{n} \mathcal{O}_{F} \rightarrow H_{n}^{D}$, as group schemes, equivariant for the $\mathcal{O}_{F}$-action.

We define $\mathfrak{I G}_{n, r} \rightarrow \mathfrak{Y}_{r}$ to be the formal scheme given by the normalization of $\mathfrak{Y}_{r}$ in $\mathcal{I} \mathcal{G}_{n, r}$. See [3, $\left.\$ 3.2\right]$ for details. Such morphism is finite and is endowed with an action of $\left(\mathcal{O}_{F} / p^{n} \mathcal{O}_{F}\right)^{*}$. One then gets a sequence of finite, $\left(\mathcal{O}_{F} / p^{r+k} \mathcal{O}_{F}\right)^{*}$-equivariant morphisms

$$
\mathfrak{I G}_{r+k, r} \rightarrow \mathfrak{I G}_{r+k-1, r} \rightarrow \cdots \rightarrow \mathfrak{Y}_{r} .
$$

The morphisms $h: \mathfrak{I G}_{n, r} \rightarrow \mathfrak{I G}_{n-1, r}$ are finite and étale over $\mathcal{Y}_{r}$. In particular, there is a trace map $\operatorname{Tr}_{\mathfrak{I G}}: h_{*} \mathcal{O}_{\mathfrak{I} \mathfrak{G}_{n, r}} \rightarrow \mathcal{O}_{\mathfrak{I} \mathfrak{G}_{n-1, r}}$.

\subsubsection{Ramification}

Proposition 3.4 We have

$$
\operatorname{Hdg} p^{n-1} \mathcal{O}_{\mathfrak{I} \mathfrak{G}_{n-1, r}} \subset \operatorname{Tr}_{\mathfrak{I G}}\left(h_{*} \mathcal{O}_{\mathfrak{I} \mathfrak{G}_{n, r}}\right)
$$

for every $1 \leq n \leq r+k$.

$$
\text { Moreover, if } p \text { is unramified one has } \operatorname{Tr}_{\mathfrak{I} \mathfrak{G}}\left(h_{*} \mathcal{O}_{\mathfrak{I} \mathfrak{G}_{1, r}}\right)=\mathcal{O}_{\mathfrak{Y}_{r}} \text {. }
$$

Proof The claim for $n \geq 2$ follows arguing as in [3, Prop. 3.4]. We recall the argument. By normality the natural map $\mathcal{I G}_{n, r} \rightarrow H_{n}^{D}$ over $\mathcal{Y}_{r}$, associated with an isomorphism $\mathcal{O}_{F} / p^{n} \mathcal{O}_{F} \rightarrow H_{n}^{D}$ the image of $1 \in \mathcal{O}_{F} / p^{n} \mathcal{O}_{F}$, extends to a morphism of formal schemes 
$\mathfrak{I G}_{n, r} \rightarrow H_{n}^{D}$ over $\mathfrak{Y}_{r}$. In particular, we get a commutative diagram of formal schemes over $\mathfrak{Y}_{r}$ :

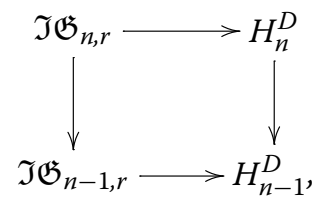

which is cartesian over the analytic fiber $\mathcal{Y}_{r}$. In particular, $\mathfrak{I G}_{n, r} \rightarrow \mathfrak{I G}_{n-1, r}$ is the normalization of the fppf $\left(H_{n} / H_{n-1}\right)^{D}$-torsor over $\mathfrak{I G}_{n-1, r}$ obtained by the fiber product of the diagram above. One reduces to prove the claimed result for the trace of the morphism $H_{n}^{D} \rightarrow H_{n-1}^{D}\left(\right.$ over $\left.\mathfrak{Y}_{r}\right)$, and this follows from the relation between the different and the trace and a careful analysis of the different of $\left(H_{n} / H_{n-1}\right)^{D}$ given in [3, Cor. A.2].

We are left to discuss the case $n=1$. If $p$ is unramified, then the degree of $\mathcal{I} \mathcal{G}_{1, r} \rightarrow \mathcal{Y}_{r}$ is prime to $p$ and the second claim of the proposition follows immediately. If $p$ is ramified, we let $\mathfrak{p}$ be the product of all primes of $\mathcal{O}_{F}$ over $p$. We introduce a variant of $\mathcal{I} \mathcal{G}_{1, r}$ by setting $\mathcal{I} \mathcal{G}_{1, r}^{\prime}$ to be the adic space over $\mathcal{Y}_{r}$ classifying isomorphisms $\mathcal{O}_{F} / \mathfrak{p} \mathcal{O}_{F} \rightarrow H_{1}[\mathfrak{p}]^{D}$, as group schemes, equivariant for the $\mathcal{O}_{F}$-action. Here $H_{1}[\mathfrak{p}]$ is the kernel of multiplication by $\mathfrak{p}$ on $H_{1}$.

We have a natural map of adic spaces $\mathcal{I}_{1, r} \rightarrow \mathcal{I G}_{1, r}^{\prime} \rightarrow \mathcal{Y}_{r}$. Taking normalizations, we get morphisms of formal schemes $\mathfrak{I G}_{1, r} \rightarrow \mathfrak{I G}_{1, r}^{\prime} \rightarrow \mathfrak{Y}_{r}$.

The degree of $\mathcal{I G}_{1, r}^{\prime} \rightarrow \mathcal{Y}_{r}$ is the order of $\left(\mathcal{O}_{F} / \mathfrak{p} \mathcal{O}_{F}\right)^{*}$ which is prime to $p$ so that $\operatorname{Tr}_{\mathfrak{I G}}\left(h_{*} \mathcal{O}_{\mathfrak{I} \mathfrak{G}_{1, r}^{\prime}}\right)=\mathcal{O}_{\mathfrak{Y}_{r}}$. We are left to estimate the image of the trace map associated with the morphism $\mathfrak{I G}_{1, r} \rightarrow \mathfrak{I} \mathfrak{G}_{1, r}^{\prime}$. Arguing as at the beginning of the proof we get a commutative diagram of formal schemes over $\mathfrak{Y}_{r}$, which is cartesian over $\mathcal{Y}_{r}$ :

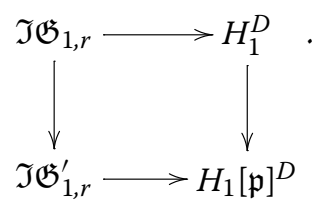

Thus $\mathfrak{I G}_{1, r}$ is the normalization of a torsor under $\left(H_{1} / H_{1}[\mathfrak{p}]\right)^{D}$. We have an exact sequence $0 \rightarrow\left(H_{1} / H_{1}[\mathfrak{p}]\right)^{D} \rightarrow H_{1}^{D} \rightarrow\left(H_{1}[\mathfrak{p}]\right)^{D} \rightarrow 0$. It follows that Hdg is contained in the different of $\left(H_{1} / H_{1}[\mathfrak{p}]\right)^{D}$ over $\mathfrak{Y}_{r}$ and we conclude.

We immediately get the following

Corollary 3.5 Let Spf $R$ be an open of $\mathfrak{Y}_{r}$ such that the ideal sheaf $\mathrm{Hdg}$ is trivial and choose a generator Ha. For every $0 \leq n \leq r+k$ there exist elements $c_{0}=1$ and $c_{n} \in$ $\tilde{H a}^{-\frac{p^{n}-1}{p-1}} \mathcal{O}_{\mathfrak{I} \mathfrak{G}_{n, r}}(\operatorname{Spf} R)$ for $n \geq 1$ such that $\operatorname{Tr}_{\mathfrak{I G}}\left(c_{n}\right)=c_{n-1}$ for every $n \geq 1$.

\subsubsection{Frobenius}

Recall from Proposition 3.3 that we have a Frobenius map $\phi: \mathfrak{Y}_{r} \rightarrow \mathfrak{Y}_{r-1}$.

Proposition 3.6 There exists an $\left(\mathcal{O}_{F} / p^{n} \mathcal{O}_{F}\right)^{*}$-equivariant map $\phi: \mathfrak{I G}_{n, r} \rightarrow \mathfrak{I G}_{n, r-1}$ lifting the map $\phi: \mathfrak{Y}_{r} \rightarrow \mathfrak{Y}_{r-1}$.

Proof As $\mathfrak{I G}_{n, r}$ is constructed by normalizing $\mathfrak{Y}_{r}$ in $\mathcal{I} \mathcal{G}_{n, r}$, it suffices to construct a lift $\phi: \mathcal{I G}_{n, r} \rightarrow \mathcal{I G}_{n, r^{\prime}}$ at the level of adic spaces. 
Notice that $H_{n+1} / H_{1}$ is the canonical subgroup $H_{n}^{\prime}$ of level $n$ of $G^{\prime}=G / H_{1}$ thanks to [3, cor. A.2]. As multiplication by $p$ on $H_{n+1}$ defines an isomorphism $H_{n+1} / H_{1} \cong H_{n}$ and hence an isomorphism $H_{n} \cong H_{n}^{\prime}\left(\operatorname{over} \mathcal{Y}_{r}\right)$. Any $\mathcal{O}_{F}$-linear isomorphism map $\mathcal{O}_{F} / p^{n} \mathcal{O}_{F} \rightarrow$ $H_{n}^{D}$ defines an $\mathcal{O}_{F}$-linear isomorphism $\Psi^{\prime}: \mathcal{O}_{F} / p^{n} \mathcal{O}_{F} \rightarrow\left(H_{n}^{\prime}\right)^{D}$.

\subsection{The basic constructions}

Recall from Sect. 2.2 that we have introduced an $\mathfrak{m}$-adic formal scheme $\tilde{\mathfrak{W}}_{F}^{0}$, which is a formal model of the weight space. It is characterized by the property that the inverse image of the maximal ideal of $\Lambda_{F}^{0}$ is an invertible ideal sheaf of $\mathcal{O}_{\widetilde{\mathfrak{W}}_{F}^{0}}$.

For every element $\alpha \in \mathfrak{m}$, we have denoted by $\mathfrak{W}_{\alpha}^{0}:=\operatorname{Spf} B_{\alpha}^{0}$ the open formal affine subscheme of $\widetilde{\mathfrak{W}}_{F}^{0}$ defined by $\alpha$. We have set $\mathcal{W}_{\alpha}^{0}:=\operatorname{Spa}\left(B_{\alpha}^{0}\left[\alpha^{-1}\right], B_{\alpha}^{0}\right)$ to be the analytic adic subspace of $\widetilde{\mathcal{W}}_{F}^{0}$ defined by $\mathfrak{W}_{\alpha}^{0}$.

Applying the construction of Sect. 3.2 with $A_{0}=B_{\alpha}^{0}$, one obtains a formal scheme $\mathfrak{X}_{r, \alpha}$ over $\mathfrak{W}_{\alpha}^{0}$. Set $\mathcal{X}_{r, \alpha}$ to be the associated analytic adic space over $\mathcal{W}_{\alpha}$.

For all choices of $\alpha$, these formal schemes $\mathfrak{X}_{r, \alpha}$ glue into a formal scheme $\mathfrak{X}_{r} \rightarrow \widetilde{\mathfrak{W}}_{F}^{0}$. We let $\mathcal{X}_{r} \rightarrow \mathcal{W}_{F}^{0}$ be the analytic adic space associated with $\mathfrak{X}_{r}$.

Let $I=\left[p^{k}, p^{k^{\prime}}\right] \subset[1, \infty]$ be an interval. We defined a formal scheme $\widetilde{\mathfrak{W}}_{F, I}^{0} \rightarrow \widetilde{\mathfrak{W}}_{F}^{0}$ and now we consider $\mathfrak{X}_{r, I}=\mathfrak{X}_{r} \times \widetilde{\mathfrak{W}}_{F}^{0} \widetilde{\mathfrak{W}}_{F, I}^{0}$ and $\mathfrak{X}_{r, \alpha, I}=\mathfrak{X}_{r, \alpha} \times_{\mathfrak{W}_{\alpha}^{0}} \mathfrak{W}_{\alpha, I}^{0}$.

Let $n \in \mathbb{N}$ be an integer such that $1 \leq n \leq r+k$. Applying the considerations of Sect. 3.3, we obtain an étale cover of adic spaces

$$
\mathcal{I G}_{n, r, \alpha, I} \rightarrow \mathcal{X}_{r, \alpha, I},
$$

for the group $\left(\mathcal{O}_{F} / p^{n} \mathcal{O}_{F}\right)^{*}$, classifying the isomorphisms $\mathcal{O}_{F} / p^{n} \mathcal{O}_{F} \rightarrow H_{n}^{D}$, as group schemes, equivariant for the $\mathcal{O}_{F}$-action. This is a morphism of adic spaces associated with a morphism of formal schemes

$$
\mathfrak{I G}_{n, r, \alpha, I} \rightarrow \mathfrak{X}_{r, \alpha, I} .
$$

For various $\alpha \in \mathfrak{m}$, these adic spaces and formal schemes glue and we obtain $\mathcal{I} \mathcal{G}_{n, r, I} \rightarrow \mathcal{X}_{r, I}$ and $\mathfrak{I G}_{n, r, I} \rightarrow \mathfrak{X}_{r, I}$.

\subsubsection{Equations}

In this subsection, we give local equations for some of the spaces defined so far. We have:

$$
B_{\alpha}^{0}=\mathbb{Z}_{p} \llbracket T_{1}, \ldots, T_{g} \rrbracket\left\langle\frac{p}{\alpha}, \frac{T_{1}}{\alpha}, \ldots, \frac{T_{g}}{\alpha}\right\rangle .
$$

If $\alpha \in \mathfrak{m} \backslash \mathfrak{m}^{2}$, this is a regular ring. Otherwise, this ring is 0 . Consider an interval $I=\left[p^{k}, p^{h}\right]$ with $k \geq 0$ an integer and $h \geq k$ an integer or $h=\infty$. Take $\alpha \in \mathfrak{m} \backslash \mathfrak{m}^{2}$. If $1 \in I$ and $\alpha=p$, then $B_{\alpha, I}^{0}=B_{\alpha}$. If $\alpha=p$ and $1 \notin I, B_{\alpha, I}^{0}=0$. Assume now that $\alpha \neq p$.

1. If $h \neq \infty$, then $B_{\alpha, I}^{0}=\mathbb{Z}_{p} \llbracket T_{1}, \ldots, T_{g} \rrbracket\left\langle\frac{T_{1}}{\alpha}, \ldots, \frac{T_{g}}{\alpha}, u, v\right\rangle /\left(\alpha^{p^{k}} v-p, u v-\alpha^{p^{h-k}}\right)$,

2. If $h=\infty$, then $B_{\alpha, I}^{0}=\mathbb{Z}_{p} \llbracket T_{1}, \ldots, T_{g} \rrbracket\left\langle\frac{T_{1}}{\alpha}, \ldots, \frac{T_{g}}{\alpha}, u\right\rangle /\left(\alpha^{p^{k}} u-p\right)$.

In the second case, $B_{\alpha, I}^{0}$ is a regular ring and, in particular, it is normal. In the first case, one checks that $B_{\alpha, I}$ is normal by verifying that it is Cohen-Macaulay and regular in codimension 1 (Serre's criterion).

Let $U:=\operatorname{Spf} A$ be a formal open affine subscheme of $\overline{\mathfrak{M}}\left(\mu_{N}, \mathfrak{c}\right)$ over which $\omega_{G}$ is trivial. Let Ha be a lift of Ha. The inverse image of $U$ in $\mathfrak{X}_{r, \alpha,\left[p^{k}, \infty\right]}$ is $\operatorname{Spf} R$ with

$$
R:=A \hat{\otimes}_{\mathbb{Z}_{p}} B_{\alpha}^{0}\langle u, w\rangle /\left(w \tilde{\mathrm{Ha}}^{p^{r}+1}-\alpha, \alpha^{p^{k}} u-p\right) .
$$


Similarly for integers $0 \leq k \leq h$ the inverse image of $U$ in $\mathfrak{X}_{r, \alpha,\left[p^{k}, p^{h}\right]}$ is $\operatorname{Spf} R^{\prime}$ with

$$
R^{\prime}:=A \hat{\otimes}_{\mathbb{Z}_{p}} B_{\alpha}^{0}\langle u, v, w\rangle /\left(w \tilde{\mathrm{Ha}}^{p^{r}+1}-\alpha, \alpha^{p^{k}} u_{k}-p, u_{k} v_{h}-\alpha^{p^{h-k}}\right) .
$$

Lemma 3.7 The rings $R$ and $R^{\prime}$ are normal.

Proof The ring $B_{\alpha}^{0}\langle u, w\rangle /\left(\alpha^{p^{k}} u-p\right)$ is Cohen-Macaulay, and the algebra $A \hat{\otimes}_{\mathbb{Z}_{p}} B_{\alpha}^{0}\langle u, w\rangle /$ $\left(\alpha^{p^{k}} u-p\right)$ is $B_{\alpha}^{0}\langle u, w\rangle /\left(\alpha^{p^{k}} u-p\right)$-flat. Moreover, $A$ is Cohen-Macaulay and flat over $\mathbb{Z}_{p}$; thus $A / p A$ is also Cohen-Macaulay. As a result, $A \hat{\otimes}_{\mathbb{Z}_{p}} B_{\alpha}^{0}\langle u, w\rangle /\left(\alpha^{p^{k}} u-p\right)$ is CohenMacaulay over $B_{\alpha}^{0}\langle u, w\rangle /\left(\alpha^{p^{k}} u-p\right)$, so it is Cohen-Macaulay. Since $R$ is a complete intersection in $A \hat{\otimes}_{\mathbb{Z}_{p}} B_{\alpha}^{0}\langle u, w\rangle /\left(\alpha^{p^{k}} u-p\right)$, it is Cohen-Macaulay. Let us check that $R$ is regular in codimension 1 . Let $\mathfrak{P}$ be a codimension 1 prime ideal of $R$. Then $R_{\mathfrak{P}}$ is easily seen to be regular if $\alpha \notin \mathfrak{P}$. Assume that $\alpha$ lies in $\mathfrak{P}$. Then $\mathfrak{P}$ is a generic point of

$$
A / p A \otimes_{\mathbb{F}_{p}}\left(B_{\alpha}^{0} / \alpha B_{\alpha}^{0}\right)[u, w] /\left(w \mathrm{Ha}^{p^{r+1}}\right) .
$$

Either $\mathrm{Ha}^{p^{r+1}} \in \mathfrak{P}$ and in that case $\mathfrak{P}$ maps to the generic point $\mathfrak{P}^{\prime}$ of an irreducible component of $A /(p A, \mathrm{Ha})$. By Andreatta and Goren [1] the ring $(A / p A)_{\mathfrak{P}^{\prime}}$ is a DVR so let $t$ be a generator of its maximal ideal. If $\hat{t}$ denotes a lift of $t$ in $R$, then $\hat{t}$ is a generator of the maximal ideal of $R_{\mathfrak{P}}$ and we are done. Otherwise, $w \in \mathfrak{P}$ and in that case $\mathfrak{P}$ maps to a generic point of $A / p A$, and $w$ is a generator of the maximal ideal of $R_{\mathfrak{P}}$. The normality of the ring $R^{\prime}$ follows along similar lines.

Corollary 3.8 The formal schemes $\mathfrak{X}_{r,\left[p^{k}, p^{h}\right]}$ are normal.

\section{Overconvergent modular sheaves in characteristic 0}

In this section, we will construct sheaves of overconvergent Hilbert modular forms over the adic space $\mathcal{W}_{F} \backslash\{|p|=0\}$. This was already accomplished in [2], but our goal now is to provide canonical integral models for the modular sheaves constructed in [2].

\subsection{A modified integral structure on $\omega_{G}$}

Fix an interval $I=\left[p^{k}, p^{k^{\prime}}\right]$ with $k$ and $k^{\prime}$ integers such that $k^{\prime} \geq k \geq 0$. Let $r \in \mathbb{Z}_{\geq 1}$ and fix a positive integer $n$ with $n \leq r+k$. Let $G$ be the semi-abelian scheme over $\mathfrak{X}_{r, I}$. It follows from Proposition 3.2 that there exists a canonical subgroup $H_{n} \subset G\left[p^{n}\right]$.

Let $g_{n}: \mathfrak{I G}_{n, r, I} \rightarrow \mathfrak{X}_{r, I}$ be the partial Igusa tower defined in Sect. 3.4. Let $\omega_{G}$ be the sheaf of invariant differentials of $G$. It follows from [3, Cor. A.2] that the kernel of the map $\omega_{G} / p^{n} \omega_{G} \rightarrow \omega_{H_{n}}$ is annihilated by $\operatorname{Hdg}^{\frac{p^{n}-1}{p-1}} \omega_{G}$. We deduce that the projection map $\omega_{G} \rightarrow \omega_{G} / p^{n} \mathrm{Hdg}^{-\frac{p^{n}-1}{p-1}} \omega_{G}$ factors via $\omega_{H_{n}}$. One then has a commutative diagram of $f p p f$ sheaves of abelian groups over $\mathfrak{X}_{r, I}$ :

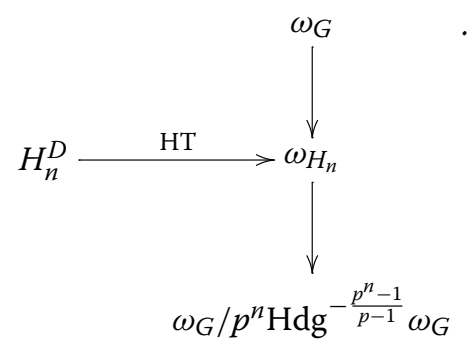

where all vertical arrows are surjective and the horizontal arrow is the Hodge-Tate map. 
Over $\mathfrak{I G}_{n, r, I}$ we have a universal section $P \in H_{n}^{D}$ which is the image of 1 via the universal morphism $\psi_{n}: \mathcal{O}_{F} / p^{n} \mathcal{O}_{F} \rightarrow H_{n}^{D}$.

Proposition 4.1 Let $\mathcal{F}$ be the inverse image in $\omega_{G}$ of the $\mathcal{O}_{\mathfrak{I} \mathfrak{G}_{n, r, I}}$-submodule of $\omega_{G} / p^{n} \mathrm{Hdg}^{-\frac{p^{n}-1}{p-1}} \omega_{G}$ spanned by $\operatorname{HT}(P)$. Then $\mathcal{F}$ is a locally free $\mathcal{O}_{F} \otimes \mathcal{O}_{\mathfrak{I} \mathfrak{G}_{n, r}, \text {-module }}$ of rank 1 , the cokernel of $\mathcal{F} \subset \omega_{G}$ is annihilated by $\mathrm{Hdg}^{\frac{1}{p-1}}$, and the map $\mathrm{HT} \circ \psi_{n}$ defines an isomorphism of $\mathcal{O}_{F} \otimes \mathcal{O}_{\mathfrak{I} \mathfrak{G}_{h, r}, I}$-modules:

$$
\mathrm{HT}^{\prime}: \mathcal{O}_{F} \otimes \mathcal{O}_{\mathfrak{I G}_{n, r, I}} / p^{n} \mathrm{Hdg}^{-\frac{p^{n}}{p-1}} \mathcal{O}_{\mathfrak{I} \mathfrak{G}_{h, r, I}} \cong \mathcal{F} / p^{n} \mathrm{Hdg}^{-\frac{p^{n}}{p-1}} \mathcal{F}
$$

Proof This is a variant of [2, Prop. 3.4]. Let $U:=\operatorname{Spf} R \subset \mathfrak{I G}_{n, r, I}$ be an open formal affine subscheme such that $\left.\omega_{G}\right|_{U}$ is free of rank $g$ as an $R$-module. Write $\bar{M} \in \mathrm{M}_{n \times n}\left(R / p^{n} \mathrm{Hdg}^{-\frac{p^{n}-1}{p-1}} R\right)$ for the matrix of the linearization of the map HT over $U$. Thanks to [3, prop. A.3] it has determinant ideal equal to $\mathrm{Hdg}^{\frac{1}{p-1}}$. In particular, $\operatorname{Hdg}^{\frac{1}{p-1}} \omega_{G} / p^{n} \operatorname{Hdg}^{-\frac{p^{n}-1}{p-1}} \omega_{G}$ lies in the span of $\operatorname{HT}(P)$.

Let $M \in \mathrm{M}_{n \times n}(R)$ be any lift of $\bar{M}$. Its determinant $\delta$ is $\operatorname{Hdg}^{\frac{1}{p-1}}$ (up to unit). Let $\left.\mathcal{S} \subset \omega_{G}\right|_{U}$ be the submodule spanned by the columns of $M$. Then $\delta \omega_{G} \subset \mathcal{S}$. Since $p^{n} \operatorname{Hdg}^{-\frac{p^{n}-1}{p-1}} \omega_{G}=p^{n} \operatorname{Hdg}^{-\frac{p^{n}}{p-1}} \cdot \delta \omega_{G} \subset \mathcal{S}$ one deduces that $\left.\mathcal{F}\right|_{U}$ coincides with the $\mathcal{S}$. In particular, it is a free $R$-module of rank $g$. By definition it is stable for the action of $\mathcal{O}_{F}$.

For every $x \in \mathcal{O}_{F} / p^{n} \mathcal{O}_{F}$, the image $y \in \mathrm{HT}\left(\psi_{n}(x)\right)$ lies by construction in the image of $\mathcal{S}$ in $\omega_{G} / p^{n} \operatorname{Hdg}^{-\frac{p^{n}-1}{p-1}} \omega_{G}$. As $\operatorname{Hdg}^{-\frac{p^{n}}{p-1}}=\operatorname{Hdg}^{-\frac{p^{n}-1}{p-1}} \cdot \operatorname{Hdg}^{\frac{1}{p-1}}$ and as $\omega_{G} / \mathcal{F}$ is annihilated by $\operatorname{Hdg} \frac{1}{p-1}$, it follows that any two lifts $y^{\prime}$ and $y^{\prime \prime}$ in $\mathcal{S}$ differ by an element lying in $\operatorname{Hdg}^{-\frac{p^{n}-1}{p-1}} \cdot \operatorname{Hdg}^{\frac{1}{p-1}} \omega_{G}=\operatorname{Hdg}^{-\frac{p^{n}-1}{p-1}} \cdot \mathcal{S}$. We then get a well-defined map $\mathcal{O}_{F} / p^{n} \mathcal{O}_{F} \rightarrow \mathcal{F} / p^{n} \mathrm{Hdg}^{-\frac{p^{n}}{p-1}} \mathcal{F}$ inducing $\mathrm{HT} \circ \psi_{n}$ when composed with the projection to $\omega_{G} / p^{n} \mathrm{Hdg}^{-\frac{p^{n}}{p-1}} \mathcal{F} \omega_{G}$. This provides the $\mathrm{HT}^{\prime}$. By construction, its restriction to $U$ is a surjective map of free $R / p^{n} \mathrm{Hdg}^{-\frac{p^{n}}{p-1}} R$-modules of rank $g$ and hence it is an isomorphism. It follows that $\mathcal{S}=\left.\mathcal{F}\right|_{U}$ is a free $\mathcal{O}_{F} \otimes R$-module of rank 1 concluding the proof of the proposition.

We denote by $f_{n}: \mathfrak{F}_{n, r, I} \rightarrow \mathfrak{I G}_{n, r, I}$ the torsor for the group $1+p^{n} \operatorname{Hdg}^{-\frac{p^{n}}{p-1}} \operatorname{Res}_{\mathcal{O}_{F} / \mathbb{Z}} \mathbb{G}_{a}$ defined by

$$
\mathfrak{F}_{n, r, I}(R):=\left\{\omega \in \mathcal{F}, \omega=\operatorname{HT}^{\prime}(1) \quad \text { in } \quad \mathcal{F} / p^{n} \operatorname{Hdg}^{-\frac{p^{n}-1}{p-1}} \mathcal{F}\right\} .
$$

One has an action of $\left(\mathcal{O}_{F} \otimes \mathbb{Z}_{p}\right)^{*}$ on $\mathfrak{F}_{n, r, I}$, lifting the action of $\left(\mathcal{O}_{F} / p^{n} \mathcal{O}_{F}\right)^{*}$ on $\mathfrak{I}_{n, r, I}$, given by $\lambda \cdot(\omega, 1)=(\lambda \omega, \lambda)$. We then get a well-defined action of the group $\left(\mathcal{O}_{F} \otimes \mathbb{Z}_{p}\right)^{*}$. $\left(1+p^{n} \operatorname{Hdg}^{-\frac{p^{n}}{p-1}} \operatorname{Res}_{\mathcal{O}_{F} / \mathbb{Z}} \mathbb{G}_{a}\right)$ on $\mathfrak{F}_{n, r, I}$.

\subsection{The sheaves of overconvergent forms}

Fix an interval $I=\left[p^{k}, p^{k^{\prime}}\right]$ with $k$ and $k^{\prime}$ integers such that $k^{\prime} \geq k \geq 0$. Let $r, n \in \mathbb{Z}_{\geq 0}$. We assume that $r \geq 3, r+k \geq n \geq k^{\prime}+2$ (resp. $r+k \geq n \geq k^{\prime}+4$ if $p=2$ ). Set $n^{\prime}=n-k^{\prime}-2\left(\right.$ resp. $n^{\prime}=n-k^{\prime}-4$ if $\left.p=2\right)$.

Lemma 4.2 We have $p \mathrm{Hdg}^{-p^{n}} \subset \mathcal{O}_{\mathfrak{I} \mathfrak{G}_{n, r}, I}$. In particular, $p^{n} \mathrm{Hdg}^{-\frac{p^{n}}{p-1}} \subset p^{k^{\prime}+1} \mathcal{O}_{\mathfrak{I} \mathfrak{G}_{h, r, I}}$ (resp. $\subset p^{k^{\prime}+3} \mathcal{O}_{\mathfrak{I G}_{h, r, I}}$ if $p=2$ ). 
Proof The claim is local on $\mathfrak{I G}_{h, r, I}$. Let $\alpha \in \mathfrak{m} \backslash \mathfrak{m}^{2}$. We prove the claim over an open $U=\operatorname{Spf} R \subset \mathfrak{I}_{h, r, \alpha, I}$ over the open $\mathfrak{W}_{\alpha, I}^{0}=\operatorname{Spf} B_{\alpha, I}^{0}$ of $\widetilde{\mathfrak{W}}_{F}^{0}$. By construction, we have $p / \alpha^{p^{k}} \in B_{\alpha, I}^{0}$ and $\alpha \mathrm{Hdg}^{-p^{r+1}} \subset R$. Hence, $p \mathrm{Hdg}^{-p^{r+k+1}} \subset R$. In particular, $p \mathrm{Hdg}^{-p^{n}} \subset R$ and the second claim follows.

Proposition 2.8 implies that the character $\kappa$ extends to a character

$$
\kappa:\left(\mathcal{O}_{F} \otimes \mathbb{Z}_{p}\right)^{*} \cdot\left(1+p^{n} \operatorname{Hdg}^{-\frac{p^{n}}{p-1}} \operatorname{Res}_{\mathcal{O}_{F} / \mathbb{Z}} \mathbb{G}_{a}\right) \rightarrow \mathbb{G}_{m}
$$

over $\mathfrak{W}_{F, I}^{0}$.

Define $\mathfrak{w}_{n, r, I}^{1}=f_{n, *} \mathcal{O}_{\mathfrak{F}_{n, r}, I}\left[\kappa^{-1}\right]$ as the subsheaf of $f_{n, *} \mathcal{O}_{\mathfrak{F}_{n, r}, I}$ of sections transforming according to the character $\kappa^{-1}$ under the action of $1+p^{n} \operatorname{Hdg}^{-\frac{p^{n}}{p-1}} \operatorname{Res}_{\mathcal{O}_{F} / \mathbb{Z}} \mathbb{G}_{a}$. It is an invertible sheaf over $\mathfrak{I}^{\mathfrak{G}_{n, r, I}}$. Define $\mathfrak{w}_{n, r, I} \subset g_{n, *} \mathfrak{w}_{n, r, I}^{1}$ as the subsheaf of $\left(g_{n} \circ f_{n}\right)_{*} \mathcal{O}_{\mathfrak{F}_{n, r, I}}$ of $\kappa^{-1}$-equivariant sections for the action of $\left(\mathcal{O}_{F} \otimes \mathbb{Z}_{p}\right)^{*} \cdot\left(1+p^{n} \operatorname{Hdg}^{-\frac{p^{n}}{p-1}} \operatorname{Res}_{\mathcal{O}_{F} / \mathbb{Z}} \mathbb{G}_{a}\right)$.

Proposition 4.3 The sheaf $\mathfrak{w}_{h, r, I}$ is an invertible $\mathcal{O}_{\mathfrak{X}_{r, I}}$-module of rank 1.

The rest of this section is devoted to the proof of Proposition 4.3. We follow closely $[3, \$ 5]$ by starting with the following:

Lemma 4.4 Let $\left(\mathcal{O}_{\mathfrak{X}_{r, I}}\right)^{00}$ be the ideal of topologically nilpotent elements of $\mathcal{O}_{\mathfrak{X}_{r, I}}$. Suppose that $r \geq 1$ (resp. $r \geq 2$ if $p=2$ ). Then $\kappa\left(\left(\mathcal{O}_{F} \otimes \mathbb{Z}_{p}\right)^{*}\right)-1 \subset \operatorname{Hdg}\left(\mathcal{O}_{\mathfrak{X}_{r, I}}\right)^{00}$ and for every integer $\ell$ such that $2 \leq \ell \leq r+k$ we have

$$
\kappa\left(1+p^{\ell-1} \mathcal{O}_{F} \otimes \mathbb{Z}_{p}\right)-1 \subset \operatorname{Hdg}^{\frac{p^{\ell}-1}{p-1}}\left(\mathcal{O}_{\mathfrak{x}_{r, I}}\right)^{00} .
$$

Proof We deal with the case $p \neq 2$ leaving to the reader the case $p=2$. The claim is local on $\mathfrak{X}_{r, I}$. We restrict ourselves to an open formal affine subscheme $U=\operatorname{Spf} R$ mapping to the open $\mathfrak{W}_{\alpha}^{0}$ of $\tilde{\mathfrak{W}}_{F}^{0}$ defined by an element $\alpha \in \mathfrak{m} \backslash \mathfrak{m}^{2}$. By construction, $\kappa\left(\left(\mathcal{O}_{F} \otimes \mathbb{Z}_{p}\right)^{*}\right)-1 \subset \alpha B_{\alpha}^{0}$ and since $\alpha \mathrm{Hdg}^{-1} \subset\left(\mathcal{O}_{\mathfrak{X}_{r, I}}\right)^{00}$, we can conclude that the first point holds. Using Lemma 2.5 , we see that for $\ell \geq 2$, we have that $\kappa\left(1+p^{\ell-1} \mathcal{O}_{F} \otimes \mathbb{Z}_{p}\right)-1 \subset$ $\left(\alpha^{p^{\ell-2}}, p\right) B_{\alpha}^{0}$. Arguing as in Lemma 4.2 we deduce from the assumption that $\ell \leq r+k$ that $p \mathrm{Hdg}^{-p^{\ell}} \in\left(\mathcal{O}_{\mathfrak{X}_{r, I}}\right)^{00}$. On the other hand as $r \geq 1$, then $\alpha \in \operatorname{Hdg}^{p^{2}} \mathcal{O}_{\mathfrak{X}_{r, I}}$ so that $\alpha^{p^{\ell-2}} \in \operatorname{Hdg}^{p^{\ell}} \mathcal{O}_{\mathfrak{X}_{r, I}}$. As $\frac{p^{\ell}-1}{p-1}<p^{\ell}$, it follows that $\alpha^{p^{\ell-2}} \operatorname{Hdg}^{-\frac{p^{\ell}-1}{p-1}} \subset\left(\mathcal{O}_{\mathfrak{X}_{r, I}}\right)^{00}$.

We also have the following:

Lemma 4.5 The inclusion $\mathcal{O}_{\mathfrak{I} \mathfrak{G}_{n, r, I}} \rightarrow f_{n, *} \mathcal{O}_{\mathfrak{F}_{n, r, I}}$ defines an isomorphism

$$
\mathcal{O}_{\mathfrak{I} \mathfrak{G}_{n, r, I} / q p^{n^{\prime}}} \mathcal{O}_{\mathfrak{I} \mathfrak{G}_{n, r, I}} \rightarrow \mathfrak{w}_{n, r, I}^{1} / q p^{n^{\prime}} \mathfrak{w}_{n, r, I}^{1}
$$

Proof Consider an open formal affine subscheme $U=\operatorname{Spf} R \subset \mathfrak{I G}_{n, r, I}$ mapping to the open formal subscheme $\mathfrak{W}_{\alpha}^{0}$ of $\widetilde{\mathfrak{W}}_{F}^{0}$ defined by some $\alpha \in \mathfrak{m} \backslash \mathfrak{m}^{2}$. Assume that $\left.\omega_{G}\right|_{U}$ is free. The choice of an element $\left.\tilde{s} \in \mathcal{F}\right|_{U}$ lifting $s:=\operatorname{HT}^{\prime}(1)$ defines a section of the morphism $\left.\left.\mathfrak{F}_{n, r, I}\right|_{U} \cong \mathfrak{I G}_{n, r, I}\right|_{U}$ and hence an isomorphism $f_{\tilde{s}}:\left.\left.\mathfrak{w}_{n, r, I}^{1}\right|_{U} \rightarrow \mathcal{O}_{\mathfrak{I} \mathfrak{G}_{n, r}, I}\right|_{U}$ given by evaluating the functions at $\tilde{s}$.

Two different lifts $\tilde{s}$ and $\tilde{s}^{\prime}$ differ by an element of $1+p^{n} \operatorname{Hdg}^{-\frac{p^{n}}{p-1}} \mathcal{O}_{F} \otimes R$ thanks to Proposition 4.1. Proposition 4.2 implies that $1+p^{n} \mathrm{Hdg}^{-\frac{p^{n}}{p-1}} \mathcal{O}_{F} \otimes R \subset 1+p^{k^{\prime}+1+n^{\prime}} \mathcal{O}_{F} \otimes R$ (resp. $1+p^{k^{\prime}+3+n^{\prime}} \mathcal{O}_{F} \otimes R$ if $p=2$ ). As $I=\left[p^{k}, p^{k^{\prime}}\right]$ we conclude that $\kappa\left(1+p^{k^{\prime}+1+n^{\prime}} \mathcal{O}_{F} \otimes\right.$ 
$R) \subset 1+p^{n^{\prime}+1} R$ (and similarly $\kappa\left(1+p^{k^{\prime}+3+n^{\prime}} \mathcal{O}_{F} \otimes R\right) \subset 1+q p^{n^{\prime}} R$ for $\left.p=2\right)$. Thus $f_{\tilde{s}} \equiv f_{\tilde{s}^{\prime}}$ modulo $q p^{n^{\prime}}$. This provides the inverse to the isomorphism in the lemma.

Let $U=\operatorname{Spf} R$ be an open affine formal subscheme of $\mathfrak{X}_{r, I}$. Suppose that $\omega_{G}$ is free over $U$. Thanks to Corollary 3.5 for every nonnegative integer $n$ such that $0 \leq n \leq r+k$ there exist elements $c_{0}=1$ and $c_{n} \in \mathrm{Ha}^{-\frac{p^{n}-1}{p-1}} \mathcal{O}_{\mathfrak{I} \mathfrak{G}_{n, r}}(\operatorname{Spf} R)$ for $n \geq 1$ such that $\operatorname{Tr}_{\mathfrak{I} \mathfrak{G}}\left(c_{n}\right)=c_{n-1}$ for every $n \geq 1$. If $n$ satisfies $r+k \geq n \geq k^{\prime}+3$ (resp. $n \geq k^{\prime}+4$ if $p=2$ ), we define a projector:

$$
\begin{aligned}
e_{c_{n}}: g_{n, *} \mathfrak{w}_{n, r, I}^{1}(R) & \rightarrow \tilde{\mathrm{Ha}}^{-\frac{p^{n}-1}{p-1}} \mathfrak{w}_{n, r, I}(R) \\
s & \mapsto \sum_{\sigma \in\left(\mathcal{O}_{F} / p^{n} \mathcal{O}_{F}\right)^{*}} \kappa(\sigma) \sigma\left(c_{n} s\right)
\end{aligned}
$$

The following lemma proves Proposition 4.3:

Lemma 4.6 Let $s \in g_{n, *} \mathfrak{w}_{n, r, I}^{1}(R)$ be an element such that $s \equiv 1 \quad \bmod p$ (in the sense of Lemma 4.5). Then $e_{c_{n}}(s) \in \mathfrak{w}_{n, r, I}(R)$ and $\mathfrak{w}_{n, r, I}(R)$ is the free $R$-module generated by $e_{c_{n}}(s)$.

Proof The proof is entirely analogous to the proof of [3, Lemme 5.4]. Write $s=1+p h$ for a section $h \in \mathfrak{F}_{n, r, I}(R)$. We get

$$
e_{c_{n}}(s)=\sum_{\sigma \in\left(\mathcal{O}_{F} / p^{n} \mathcal{O}_{F}\right)^{*}} \kappa(\tilde{\sigma}) \tilde{\sigma}\left(c_{n}\right)+p \sum_{\sigma \in\left(\mathcal{O}_{F} / p^{n} \mathcal{O}_{F}\right)^{*}} \kappa(\tilde{\sigma}) \tilde{\sigma}\left(c_{n} h\right) .
$$

In this formula, $\tilde{\sigma}$ is an arbitrary lift of $\sigma$ to $\mathbb{T}\left(\mathbb{Z}_{p}\right)$. Since $\tilde{\mathrm{Ha}}{ }^{p^{r+k+1}} \mid p$ and $\frac{p^{n}-1}{p-1}<$ $p^{r+k+1}$, it follows that $p \sum_{\sigma \in\left(\mathcal{O}_{F} / p^{n} \mathcal{O}_{F}\right)^{*}} \kappa(\tilde{\sigma}) \tilde{\sigma}\left(c_{n} h\right) \in R^{00} \mathfrak{F}_{n, r, I}(R)$ where $R^{00}$ is the ideal of topologically nilpotent elements in $R$.

We need to show that

$$
\sum_{\sigma \in\left(\mathcal{O}_{F} / p^{n} \mathcal{O}_{F}\right)^{*}} \kappa(\tilde{\sigma}) \tilde{\sigma}\left(c_{n}\right) \in 1+R^{00} \mathfrak{F}_{n, r, I}(R) .
$$

This follows from Lemmas 2.6 and 4.4. As a consequence, $e_{c_{n}}(s)$ belongs to $\mathfrak{w}_{n, r, I}(R)$ and one checks easily that it is a generator using the normality of $R$ as in [3, Lemme 5.4].

\subsection{Properties of $\mathfrak{w}_{n, r, l}$}

\subsubsection{Functoriality}

Fix intervals $I^{\prime} \subset I, r^{\prime}$ and $r$ such that $r^{\prime} \geq r$ and integers $n^{\prime} \geq n$ so that $\left(I^{\prime}, r^{\prime}, n^{\prime}\right)$ and $(I, r, n)$ satisfy the assumptions given at the beginning of Sect. 4.2. We have the following commutative diagram:

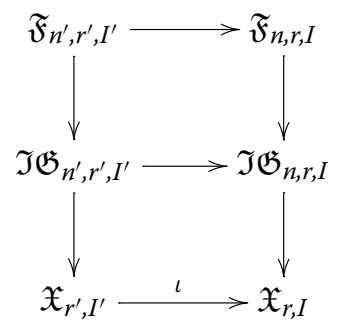

which induces a morphism of $\mathcal{O}_{\mathfrak{X}_{r^{\prime}, I^{\prime}}}$-modules:

$$
\iota^{*} \mathfrak{w}_{n, r, I} \rightarrow \mathfrak{w}_{n^{\prime}, r^{\prime}, I^{\prime}}
$$


Proposition 4.7 The morphism above is an isomorphism.

Proof Consider $\mathcal{O}_{\mathfrak{X}_{r^{\prime}, I^{\prime}}} \rightarrow \iota^{*} \mathfrak{w}_{n, r, I}^{-1} \otimes \mathfrak{w}_{n^{\prime}, r^{\prime}, I^{\prime}}$. This last sheaf is the subsheaf of $\left(g_{n^{\prime}} \circ\right.$ $\left.f_{n^{\prime}}\right)_{*} \mathcal{O}_{\mathfrak{F}_{n^{\prime}, r^{\prime}, I^{\prime}}}$ consisting of sections on which $\left(\mathcal{O}_{F} \otimes \mathbb{Z}_{p}\right)^{*} \cdot\left(1+p^{n^{\prime}} \operatorname{Hdg}^{-\frac{p^{n^{\prime}}}{p-1}} \mathcal{O}_{F} \otimes \mathbb{Z}_{p}\right)$ acts trivially. This coincides with the sheaf $\mathcal{O}_{\mathfrak{X}_{r^{\prime}, I^{\prime}}}$ by the normality of $\mathfrak{X}_{r^{\prime}, I^{\prime}}$. The composite map

$$
\mathcal{O}_{\mathfrak{X}_{r^{\prime}, I^{\prime}}} \rightarrow \iota^{*} \mathfrak{w}_{n, r, I}^{-1} \otimes \mathfrak{w}_{n^{\prime}, r^{\prime}, I^{\prime}} \rightarrow \mathcal{O}_{\mathfrak{X}_{r^{\prime}, I^{\prime}}}
$$

is the identity. This proves the claim.

We simplify the notations and write $\mathfrak{w}_{I}$ instead of $\mathfrak{w}_{n, r, I}$.

\subsubsection{Frobenius}

Propositions 3.3 and 3.6 provide compatible morphisms $\phi: \mathfrak{X}_{r, I} \rightarrow \mathfrak{X}_{r-1, I}$ and $\mathfrak{I G}_{n+1, r, I} \rightarrow \mathfrak{I G}_{n, r-1, I}$ obtained by composing the projection $\mathfrak{I G}_{n+1, r, I} \rightarrow \mathfrak{I G}_{n, r, I}$ and the Frobenius map $\phi: \mathfrak{I G}_{n, r, I} \rightarrow \mathfrak{I G}_{n, r-1, I}$. Let us recall the description of the morphism $\mathfrak{I G}_{n+1, r, I} \rightarrow \mathfrak{I G}_{n, r-1, I}$. Let $F: G \rightarrow G / H_{1}=G^{\prime}$ be the canonical isogeny between the semi-abelian schemes $G$ over $\mathfrak{X}_{r, I}$ and $G^{\prime}$ over $\mathfrak{X}_{r-1, I}$. This morphism induces a surjective morphism of canonical subgroups $H_{n+1} \rightarrow H_{n+1} / H_{1} \cong H_{n}^{\prime}$ of $G$ and $G^{\prime}$ respectively. Dualizing we get an injective morphism $F^{D}: H_{n}^{\prime D} \rightarrow H_{n+1}^{D}$. The map $\phi: \mathfrak{I G}_{n+1, r, I} \rightarrow \mathfrak{I G}_{n, r^{\prime}, I}$ associates with a morphism $\psi: \mathcal{O}_{F} / p^{n+1} \mathcal{O}_{F} \rightarrow H_{n+1}^{D}$ the morphism $\psi^{\prime}: \mathcal{O}_{F} / p^{n} \mathcal{O}_{F} \rightarrow H_{n}^{\prime} D$ making the following diagram commute:

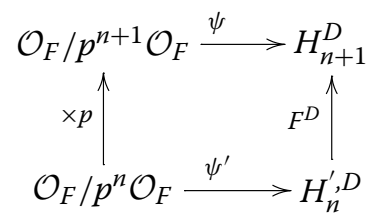

We then get the commutative diagram:

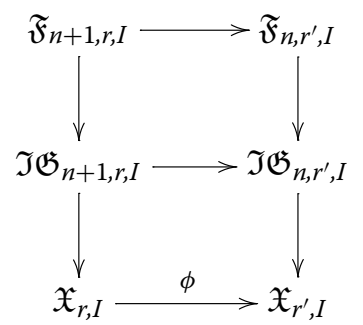

where the morphism $\mathfrak{F}_{n+1, r, I} \rightarrow \mathfrak{F}_{n, r-1, I}$ is given by mapping a differential $w \in \mathcal{F}$ to $p w \in \mathcal{F}^{\prime} \subset \omega_{G^{\prime}}$. One checks that this is well defined by using the following commutative diagram:

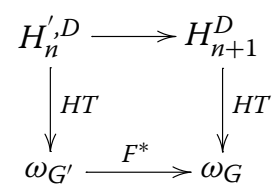

and thus obtains a morphism $\phi^{*} \mathfrak{w}_{I} \rightarrow \mathfrak{w}_{I}$.

Proposition 4.8 The morphism $\phi^{*} \mathfrak{w}_{I} \rightarrow \mathfrak{w}_{I}$ is an isomorphism.

Proof The proof is analogous to the proof of Proposition 4.7. 


\section{Perfect overconvergent modular forms}

In this section, we define a sheaf of perfect overconvergent Hilbert modular forms over the weight space $\mathfrak{W}_{F}^{0}$ and in the next we will show that one can undo the perfectization.

\subsection{The anti-canonical tower}

Let $I=\left[p^{k}, p^{k^{\prime}}\right] \subset[1,+\infty]$ and $r, n \in \mathbb{Z}_{\geq 0}$ and $n \leq r+k$. As explained in Sect. 4.3.2 we have compatible morphisms

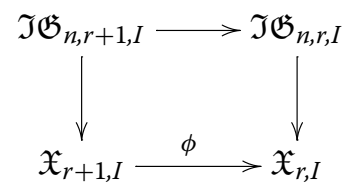

Taking the limits, we get formal schemes $\mathfrak{I G}_{n, \infty, I} \rightarrow \mathfrak{X}_{\infty, I}$ over $\widetilde{\mathfrak{W}}_{F}^{0}$. Varying $n$ we get a tower of formal schemes $\cdots \rightarrow \mathfrak{I G}_{n+2, \infty, I} \rightarrow \mathfrak{I G}_{n+1, \infty, I} \rightarrow \mathfrak{I G}_{n, \infty, I}$. Let $\mathfrak{I G}_{\infty, \infty, I}$ be the projective limit. As the index $r$ varies now, we denote by $G_{r} \rightarrow \mathfrak{X}_{r, I}$ the semi-abelian scheme and by $\operatorname{Hdg}_{r} \subset \mathcal{O}_{\mathfrak{x}_{r, I}}$ the Hodge ideal defined by $G_{r}$.

Recall from Sect. 3.3 that associated with the finite morphism $\mathfrak{I G}_{n, r, I} \rightarrow \mathfrak{I G}_{n-1, r, I}$ we have a trace map $\operatorname{Tr}_{\mathfrak{I G}}: \mathcal{O}_{\mathfrak{J G}_{h, r, I}} \rightarrow \mathcal{O}_{\mathfrak{I G}_{n-1, r, I}}$. These are compatible for varying $r$ and define a trace map $\operatorname{Tr}_{\mathfrak{I} \mathfrak{H}}: \mathcal{O}_{\mathfrak{I G}_{n, \infty, I}} \rightarrow \mathcal{O}_{\mathfrak{I G}_{n-1, \infty, I}}$.

Proposition 5.1 We have $\operatorname{Hdg}_{s} \mathcal{O}_{\mathfrak{I G} \mathfrak{G}_{n-1, \infty, I}} \subset \operatorname{Tr}_{\mathfrak{I G}}\left(\mathcal{O}_{\mathfrak{I} \mathfrak{G}_{n, \infty}, I}\right)$ for every $s \geq 1$.

Proof Thanks to Proposition 3.4, we have $\operatorname{Hdg}_{s}^{p^{n-1}} \mathcal{O}_{\mathfrak{I} \mathfrak{G}_{n-1, s, I}} \subset \operatorname{Tr}_{\mathfrak{I G}}\left(h_{*} \mathcal{O}_{\mathfrak{I} \mathfrak{G}_{n, s, I}}\right)$. It follows from [3, Cor. A.2] that $\operatorname{Hdg}_{s+1}^{p}=\operatorname{Hdg}_{s}$. Since $\operatorname{Hdg}_{s}{ }^{n-1} \mathcal{O}_{\mathfrak{I} \mathfrak{G}_{n-1, \infty, I}} \subset \operatorname{Tr}_{\mathfrak{I} \mathfrak{G}}\left(\mathcal{O}_{\mathfrak{I} \mathfrak{G}_{n, \infty, I}}\right)$ and $s$ is arbitrary, the claim follows.

\subsection{Tate traces}

Let $\alpha \in \mathfrak{m} \backslash \mathfrak{m}^{2}$. Denote by $\mathfrak{I G}_{\infty, \infty, \alpha, I} \rightarrow \mathfrak{X}_{\infty, \alpha, I}$ the base change of the formal schemes above to $\mathfrak{W}_{\alpha, I}^{0} \rightarrow \widetilde{\mathfrak{W}}_{F}^{0}$.

Let $h_{r}: \mathfrak{X}_{\infty, \alpha, I} \rightarrow \mathfrak{X}_{r, \alpha, I}$ be the projection map onto the $r$ th factor.

Proposition 5.2 One has Tate traces:

$$
\operatorname{Tr}_{r}:\left(h_{r}\right)_{*} \mathcal{O}_{\mathfrak{X}_{\infty, \alpha, I}}[1 / \alpha] \rightarrow \mathcal{O}_{\mathfrak{X}_{r, \alpha, I}}[1 / \alpha]
$$

such that $f=\lim _{r \rightarrow \infty} \operatorname{Tr}_{r}(f)$. Moreover,

$$
\operatorname{Tr}_{r}\left(\left(h_{r}\right)_{*} \mathcal{O}_{\mathfrak{X}_{\infty, \alpha, I}}\right) \subset \alpha^{-1} \cdot \mathcal{O}_{\mathfrak{X}_{r, \alpha, I}}
$$

as soon as $p^{r}(p-1)>2 g+1$.

Proof The proof follows closely the proof of [3, Proposition 6.2]. We first provide the analog of [3, \$6.3.2 and \$6.3.3] which reduces the proof to [3, Lemme 6.1].

For every nonnegative integer $k \geq r+1$, define $B_{\alpha, I, p^{-k}}^{0}:=B_{\alpha, I}^{0}\left[\alpha^{p^{-k}}\right]$. One proves as in Sect. 3.4.1 that it is a normal ring. Let $\mathfrak{W}_{\alpha, I, p^{-k}}^{0}$ be the associated $\alpha$-adic formal scheme and let $\left.\mathcal{W}_{\alpha, I, p^{-k}}^{0}:=\operatorname{Spa}\left(B_{\alpha, I, p^{-k}}^{0}\left[\alpha^{-1}\right], B_{\alpha, I, p^{-k}}^{0}\right]\right)$ be the associated analytic adic space. Define $\mathcal{X}_{r, \alpha, I, p^{-k}}$ to be the fiber product $\mathcal{X}_{r, \alpha, I} \times_{\mathcal{W}_{\alpha, I}^{0}} \mathcal{W}_{\alpha, I, p^{-k}}^{0}$. Define $\mathfrak{X}_{r, \alpha, I, p^{-(r+1)}}$ to be the normalization of $\mathfrak{X}_{r, \alpha, I}$ in $\mathcal{X}_{r, \alpha, I, p^{-(r+1)}}$ (see Sect. 3.3). For general $k \geq r+1$ let $\mathfrak{X}_{r, \alpha, I, p^{-k}}$ be the base change of $\mathfrak{X}_{r, \alpha, I, p^{-(r+1)}}$ via the map $\mathfrak{W}_{\alpha, I, p^{-k}}^{0} \rightarrow \mathfrak{W}_{\alpha, I, p^{-(r+1)}}^{0}$. The 
associated analytic adic space is $\mathcal{X}_{r, \alpha, I, p^{-k}}$ such that we have morphisms

$$
\mathfrak{X}_{r, \alpha, I, p^{-k}} \rightarrow \mathfrak{X}_{r, \alpha, I, p^{-(r+1)}} \rightarrow \mathfrak{X}_{r, \alpha, I} \rightarrow \overline{\mathfrak{M}}\left(\mu_{N}, \mathfrak{c}\right) \times \mathfrak{W}_{\alpha, I}^{0} \rightarrow \overline{\mathfrak{M}}\left(\mu_{N}, \mathfrak{c}\right) .
$$

\subsubsection{An explicit description of diagram (1) in Sect. 5.2}

Let $U:=\operatorname{Spf} A \subset \overline{\mathfrak{M}}\left(\mu_{N}, \mathfrak{c}\right)$ be an open formal affine so that the sheaf $\omega_{G}$ is trivial. We will describe the fiber of the above chain of morphisms over $U$. Choose a lift of the Hasse invariant viewed as a scalar H̃a.

The fiber of $U$ in $\mathfrak{X}_{r, \alpha, I, p^{-(r+1)}}$ is the formal spectrum of

$$
\begin{aligned}
R & :=A \widehat{\otimes} B_{\alpha, I, p^{-(r+1)}}^{0}\left\langle\frac{\alpha^{1 / p^{r+1}}}{\tilde{\mathrm{Ha}}}\right\rangle \\
& =A \widehat{\otimes} B_{\alpha, p^{-(r+1)}}^{0}\langle u, v, w\rangle /\left(w \mathrm{Ha}-\alpha^{1 / p^{r+1}}, \alpha^{p^{k}} v-p, u v-\alpha^{p^{k^{\prime}-k}}\right) .
\end{aligned}
$$

Here the variable $u$ and the equation $u v-\alpha^{p^{k^{\prime}-k}}$ are missing in case $k^{\prime}=\infty$. Arguing as in the proof of Lemma 3.7, it follows that $R$ is a normal ring.

Set $R_{k}:=R \otimes_{B_{\alpha, I, p}^{0}-(r+1)} B_{\alpha, I, p^{-k}}^{0}=A \widehat{\otimes} B_{\alpha, I, p^{-k}}^{0}\left\langle\frac{\alpha^{1 / p^{r+1}}}{\tilde{\mathrm{Ha}}}\right\rangle$. It is finite and free as an $R$-module with basis $\alpha^{a / p^{k}}$ for $0 \leq a \leq p^{k+1-r}-1$. The associated formal scheme $\operatorname{Spf} R_{k}$ is the open of $\mathfrak{X}_{r, \alpha, I, p^{-k}}$ over the open $U \subset \mathfrak{X}$.

Then the restriction of the diagram (1) to $U$ is given by the ring homomorphisms:

$$
A \rightarrow A \widehat{\otimes} B_{\alpha, I}^{0} \rightarrow A \widehat{\otimes} B_{\alpha, I}^{0}\left\langle\frac{\alpha}{\tilde{\mathrm{Ha}^{p}} p^{r+1}}\right\rangle \rightarrow R \rightarrow R_{k}
$$

\subsubsection{Frobenius}

The Frobenius morphism of Proposition 3.3 defines a cartesian diagram

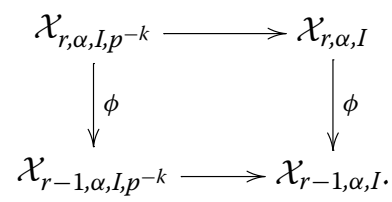

Due to Proposition 3.3, the morphism $\mathcal{X}_{r, \alpha, I, p^{-k}} \rightarrow \mathcal{X}_{r-1, \alpha, I, p^{-k}}$ is finite. Hence we get a commutative diagram

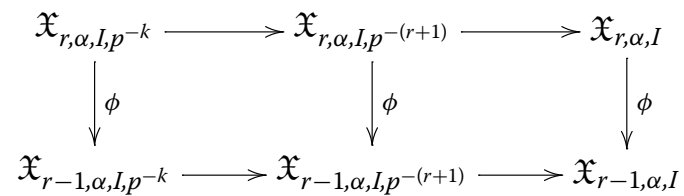

Over $U=\operatorname{Spf} A$ the morphism $\mathfrak{X}_{r, \alpha, I, p^{-k}} \rightarrow \mathfrak{X}_{r-1, \alpha, I, p^{-k}}$ is given by

$$
S_{k}:=A \widehat{\otimes} B_{\alpha, I, p^{-k}}^{0}\left\langle\frac{\alpha^{1 / p^{r}}}{\mathrm{H} \mathrm{a}}\right\rangle \rightarrow A \widehat{\otimes} B_{\alpha, I, p^{-k}}^{0}\left\langle\frac{\alpha^{1 / p^{r+1}}}{\mathrm{Ha}}\right\rangle=: R_{k} .
$$

It is finite and modulo $p \alpha^{-1 / p^{r}}$ is induced by the absolute Frobenius on $A / p A$. Indeed this holds true modulo $p \tilde{\mathrm{Ha}}^{-1}$ due to $\left[3\right.$, Cor. A.2] and $p \tilde{\mathrm{Ha}}^{-1}=\left(p \alpha^{-1 / p^{r}}\right) \cdot\left(\alpha^{1 / p^{r}} \tilde{\mathrm{Ha}}^{-1}\right)$. 


\subsubsection{The unramified case}

We first assume that $p$ is unramified in $F$. This implies that $A$ is formally smooth over $\mathbb{Z}_{p}$. It follows from [3, Lemme 6.1] applied to the extension $S_{k} \subset R_{k}$ that $S_{k}\left[\alpha^{-1}\right] \subset R_{k}\left[\alpha^{-1}\right]$ is a finite and flat extension and that $\operatorname{Tr}\left(R_{k}\right) \subset p^{g} \alpha^{-\frac{(2 g+1)}{p^{r}}} S_{k}$. This implies that for all $r^{\prime} \geq r$ and $k \geq r^{\prime}+1$,

$$
\operatorname{Tr}_{\phi^{r^{\prime}-r}}\left(\mathcal{O}_{\mathfrak{X}_{r^{\prime}, \alpha, I, p}-k}\right) \subset p^{g} \alpha^{-\frac{2 g+1}{p^{r}(p-1)}} \mathcal{O}_{\mathfrak{X}_{r, \alpha, I, p}-k} .
$$

In particular, defining

$$
\operatorname{Tr}_{r}:=\frac{1}{p^{s g}} \operatorname{Tr}_{\phi^{s}}: h_{r, *} \mathcal{O}_{\mathfrak{X}_{r+s, \alpha, I}}\left[\alpha^{-1}\right] \rightarrow \mathcal{O}_{\mathfrak{X}_{r, \alpha, I}}\left[\alpha^{-1}\right],
$$

we deduce that, if $p^{r}(p-1)>2 g+1$, the image of $h_{r, *} \mathcal{O}_{\mathfrak{X}_{r+s, \alpha, I}}$ is contained in $\alpha^{-1} \mathcal{O}_{\mathfrak{X}_{r, \alpha, I, p}-k} \cap \mathcal{O}_{\mathfrak{X}_{r, \alpha, I}}\left[\alpha^{-1}\right]$ which is $\alpha^{-1} \cdot \mathcal{O}_{\mathfrak{X}_{r, \alpha, I}}$ since $\mathfrak{X}_{r, \alpha, I, p^{-k}} \rightarrow \mathfrak{X}_{r, \alpha, I}$ is a finite and dominant morphism and $\mathfrak{X}_{r, \alpha, I}$ is normal. The proposition follows from this.

\subsubsection{The general case}

We now drop the assumption that $p$ is unramified in $F$. In this situation, $\overline{\mathfrak{M}}\left(\mu_{N}, \mathfrak{c}\right)$ is not formally smooth. Nevertheless, the Rapoport locus $\overline{\mathfrak{M}}\left(\mu_{N}, \mathfrak{c}\right)^{R} \subset \overline{\mathfrak{M}}\left(\mu_{N}, \mathfrak{c}\right)$ is the smooth locus and its complement is of codimension at least 2. We let $\mathfrak{X}_{r, \alpha, I, p^{-k}}^{R} \subset \mathfrak{X}_{r, \alpha, I, p^{-k}}$ be the open formal subscheme where the Rapoport condition holds.

Arguing as in the unramified case, we obtain a map $\operatorname{Tr}_{r}:=\frac{1}{p^{s g}} \operatorname{Tr}_{\phi^{s}}: h_{r, *} \mathcal{O}_{\mathfrak{X}_{r+s, \alpha, I}^{R}} \rightarrow$ $\alpha^{-1} \mathcal{O}_{\mathfrak{X}_{r, \alpha, I}^{R}}$.

Lemma 5.3 The formal scheme $\mathfrak{X}_{r, \alpha, I}^{R}$ is Zariski dense in $\mathfrak{X}_{r, \alpha, I}$.

Proof This follows easily from the explicit equations. Note that we crucially use here that the complement of the Rapoport locus is of codimension 1 in the non-ordinary locus of the special fiber of $\overline{\mathfrak{M}}\left(\mu_{N}, \mathfrak{c}\right)$.

Consider the following commutative diagram:

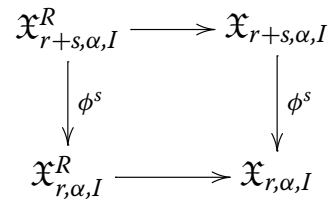

We claim that it induces a commutative diagram:

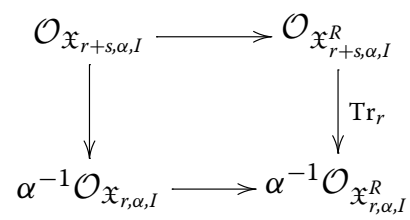

Proof Indeed, let $\operatorname{Spf} R$ be an open formal subscheme of $\mathfrak{X}_{r, \alpha, I}$. Take $f \in \mathcal{O}_{\mathfrak{X}_{r+s, \alpha, I}}(R)$. Then $\alpha \operatorname{Tr}_{r}(f)$ is in $\mathcal{O}_{\mathfrak{X}_{r, \alpha, I}^{R}}(R)$. Moreover, as the morphism $\phi^{s}: R\left[\frac{1}{\alpha}\right] \rightarrow \mathcal{O}_{\mathfrak{X}_{r, \alpha, I}}(R)\left[\frac{1}{\alpha}\right]$ is finite flat, we deduce that $\alpha \operatorname{Tr}_{r}(f) \in R\left[\frac{1}{p}\right]$. Since $R$ is normal, $R=\cap_{\mathfrak{Q}} R_{\mathfrak{Q}}$ where $\mathfrak{Q}$ runs over all codimension 1 prime ideals in $R$. Thus we are left to check that $\alpha \operatorname{Tr}_{r}(f) \in R_{\mathfrak{Q}}$ whenever $p \in \mathfrak{Q}$.

If $\alpha \notin \mathfrak{Q}$ as $w \tilde{\mathrm{Ha}}{ }^{p^{r+1}}-\alpha=0$, we deduce that the image of $\mathfrak{Q}$ in $\overline{\mathfrak{M}}\left(\mu_{N}, \mathfrak{c}\right)$ lies in the ordinary locus and in particular in the Rapoport locus. Thus $\alpha \operatorname{Tr}_{r}(f) \in R_{\mathfrak{Q}}$. If $\alpha \in \mathfrak{Q}$, then 
$\mathfrak{Q}$ is a generic point of the special fiber of $R$ and since the Rapoport locus is Zariski dense, $\mathfrak{Q}$ lies in the Rapoport locus. Thus $\alpha \operatorname{Tr}_{r}(f) \in R_{\mathfrak{Q}}$.

\subsection{The sheaf of perfect, overconvergent Hilbert modular forms}

Lemma 5.4 Let $\alpha \in \mathfrak{m} \backslash \mathfrak{m}^{2}$. The sheaf $\mathcal{O}_{\mathfrak{X}_{\infty, \alpha, I}}$ is integrally closed in $\mathcal{O}_{\mathfrak{X}_{\infty, \alpha, I}}[1 / \alpha]$. Moreover,

$$
\left(\mathcal{O}_{\Im \mathfrak{G} \infty, \infty, \alpha, I}\right)^{\left(\mathcal{O}_{F} \otimes \mathbb{Z}_{p}\right)^{*}}=\mathcal{O}_{\mathfrak{X}_{\infty, \alpha, I}}
$$

Proof (1) Let $U:=\operatorname{Spf} R$ be an open formal subscheme of $\mathfrak{X}_{\infty, \alpha, I}$. For $s$ large enough, it is the inverse image of an open formal subscheme $\operatorname{Spf} R_{s}$ of $\mathfrak{X}_{s, \alpha, I}$. Let $f \in R[1 / \alpha]$. For $s$ large enough, we have $f=f_{s}+h$ with $f_{s} \in R_{s}[1 / \alpha]$ and $h \in R$ so that we may assume that $f \in R_{s}[1 / \alpha]$. Let $f^{n}+f^{n-1} a_{n-1}+\cdots+a_{0}=0$ with $a_{0}, \ldots, a_{n-1} \in R$ be an integral relation.

Applying $\operatorname{Tr}_{s}$ one gets $f^{n}+f^{n-1} \operatorname{Tr}_{h}\left(a_{n-1}\right)+\cdots+\operatorname{Tr}_{h}\left(a_{0}\right)=0$ and as $a_{i} \in R$ for $s$ large enough we have $\operatorname{Tr}_{s}\left(a_{i}\right) \in R \cap \alpha^{-1} R_{s}$. For $h \geq 0$ the morphism $R_{s} \rightarrow R_{s+h}$ is a finite dominant morphism of normal rings. Hence $R_{s} / \alpha \rightarrow R_{s+h} / \alpha$ is injective so that $R_{s} / \alpha \rightarrow R / \alpha$ is injective as well. We deduce that $\alpha \operatorname{Tr}_{s}\left(a_{i}\right) \in \alpha R_{s}$ and hence that $\operatorname{Tr}_{s}\left(a_{i}\right) \in R_{s}$. Thus $f$ is integral over $R_{s}$ and, hence, $f \in R_{s}$ proving the first claim of the lemma.

(2) The inverse image of $\operatorname{Spf} R$ in $\mathfrak{I G}_{n, \infty, \alpha, I}$ is equal to $\operatorname{Spf} R_{n}$ with $R_{n}$ integral over $R$ and $R\left[\alpha^{-1}\right] \subset R_{n}\left[\alpha^{-1}\right]$ finite and étale. In particular, $\left(R_{n}\left[\alpha^{-1}\right]\right)^{\left(\mathcal{O}_{F} / p^{n} \mathcal{O}_{F}\right)^{*}}=R\left[\alpha^{-1}\right]$ so that $R_{n}^{\left(\mathcal{O}_{F} / p^{n} \mathcal{O}_{F}\right)^{*}}$ contains $R$ and is integral over $R$ and, hence by the first claim, it must be equal to $R$. Let $R_{\infty}$ be the inverse image of Spf $R$ in $\mathfrak{I G}_{\infty, \infty, \alpha, I}$. Consider an element $x \in R_{\infty}$ fixed by $\left(\mathcal{O}_{F} \otimes \mathbb{Z}_{p}\right)^{*}$. There exists $n$ large enough and $x_{n} \in R_{n}$ such that $x-x_{n}=\alpha x^{\prime}$ for some $x^{\prime} \in R_{\infty}$. In particular, $x^{\prime}$ is fixed by $1+p^{n} \mathcal{O}_{F} \otimes \mathbb{Z}_{p}$. Thanks to Proposition 5.1 for every $n^{\prime} \geq n$ there exists an element $c_{n^{\prime}} \in R_{n}^{\prime}$ such that $\operatorname{Tr}_{R_{n^{\prime}} / R_{n}}\left(c_{n^{\prime}}\right)=\alpha$. In particular, the higher cohomology groups of $\left(1+p^{n} \mathcal{O}_{F} / p^{n^{\prime}} \mathcal{O}_{F}\right)$ acting on $R_{n^{\prime}}$ are annihilated by $\alpha$.

For every $s$, there exists $n(s) \geq n$ such that $x^{\prime} \in\left(R_{n(s)} / \alpha^{s}\right)^{1+p^{n} \mathcal{O}_{F} \otimes \mathbb{Z}_{p}}$ and hence there exists $y_{s} \in R_{n}$ such that $y_{s} \equiv \alpha x^{\prime}$ modulo $\alpha^{s}$. We deduce that $y_{s}$ converges to an element $y$ for $s \rightarrow \infty$ such that $\alpha x^{\prime}=y$. Hence $x \in R_{n}^{\left(\mathcal{O}_{F} / p^{n} \mathcal{O}_{F}\right)^{*}}$ which is $R$ by the first part of the argument.

Define $\mathfrak{w}_{I}^{\text {perf }}$ to be the subsheaf of $\mathcal{O}_{\mathfrak{X}_{\infty, I}}$-modules of $\mathcal{O}_{\mathfrak{I} \mathfrak{G}_{\infty, \infty, I}}$ consisting of those sections transforming via the character $\kappa^{-1}$ for the action of $\left(\mathcal{O}_{F} \otimes \mathbb{Z}_{p}\right)^{*}$. Then:

Proposition 5.5 The sheaf $\mathfrak{w}_{I}^{\text {perf }}$ is an invertible $\mathcal{O}_{\mathfrak{X}_{\infty, I}}$-module. Moreover, for every subinterval $J \subset I$ the pullback of $\mathfrak{w}_{I}^{\text {perf }}$ via the natural morphism $\iota_{J, I}: \mathfrak{X}_{\infty, J} \rightarrow \mathfrak{X}_{\infty, I}$ coincides with $\mathfrak{w}_{J}^{\text {perf }}$.

Proof We prove the first claim. Let $U:=\operatorname{Spf} R$ be an open formal subscheme of $\mathfrak{X}_{\infty, \alpha, I}$. Suppose that $\operatorname{Hdg}_{1}$ is a principal ideal over $\operatorname{Spf} R$ with generator $\tilde{\mathrm{Ha}}{ }_{1}$. Let $\operatorname{Spf} R_{n}$ (resp. Spf $R_{\infty}$ ) be the inverse image of $U$ in $\mathfrak{I G}_{n, \infty, \alpha, I}$ (resp. in $\mathfrak{I} \mathfrak{G}_{\infty, \infty, \alpha, I}$ ). Due to Corollary 5.4 it suffices to exhibit an invertible element $x \in R_{\infty}$ such that $\sigma(x)=\kappa^{-1}(\sigma) x$ for every $\sigma \in\left(\mathcal{O}_{F} \otimes \mathbb{Z}_{p}\right)^{*}$.

Proposition 5.1 implies that there exist elements $c_{n} \in \tilde{\mathrm{Ha}}{ }_{1}^{-1} R_{n}$ such that $\operatorname{Tr}_{R_{n} / R_{n-1}}\left(c_{n}\right)=$ $c_{n-1}$ and $c_{0}=1$. Define $b_{n}:=\sum_{\sigma \in\left(\mathcal{O}_{F} / p^{n} \mathcal{O}_{F}\right)^{*}} \kappa(\tilde{\sigma}) \sigma\left(c_{n}\right) \in \tilde{\mathrm{Ha}}_{1}^{-1} R_{n}$ for $n \geq 1$. Here $\tilde{\sigma} \in \mathbb{T}\left(\mathbb{Z}_{p}\right)$ is a lift of $\sigma$. It follows from Lemma 2.6 that $b_{n}$ converges to an element 
$b_{\infty} \in R_{\infty}$ such that $\sigma\left(b_{\infty}\right)=\kappa^{-1}(\sigma) b_{\infty}$ for every $\sigma \in\left(\mathcal{O}_{F} \otimes \mathbb{Z}_{p}\right)^{*}$ and $b_{\infty} \equiv 1$ modulo $\frac{\alpha}{\tilde{H} a_{1}} R_{\infty}$ so that $b_{\infty}$ is invertible in $R_{\infty}$ as claimed.

The last claim can be proved as in Sect. 4.3.

\section{Descent}

In this section, we prove that the sheaf $\mathfrak{w}_{I}^{\text {perf }}$ defined in Sect. 5.3 can be descended to some finite level.

\subsection{Comparison with the sheaf $\mathfrak{w}_{I}$}

Consider an interval $I=\left[p^{k}, p^{k^{\prime}}\right]$ with $k$ and $k^{\prime}$ nonnegative integers. Thanks to Proposition 4.3, we have an invertible sheaf $\mathfrak{w}_{I}$ over $\mathfrak{X}_{r, I}$. Recall that we have a projection map $h_{r}: \mathfrak{X}_{\infty, I} \rightarrow \mathfrak{X}_{r, I}$. Then:

Proposition 6.1 There exists a canonical isomorphism $\mathfrak{w}_{I}^{\text {perf }} \simeq h_{r}^{*} \mathfrak{w}_{I}$.

Proof Over $\mathfrak{X}_{\infty, \alpha, I}$ we have a chain of isogenies

$$
\cdots G_{n+r} \stackrel{F}{\rightarrow} G_{n+r-1} \rightarrow \cdots G_{r}
$$

where $G_{s}$ is the versal semi-abelian scheme over $\mathfrak{X}_{s, I}$. Denote by $C_{n, r} \hookrightarrow G_{r}\left[p^{n}\right]$ the kernel of $\left.\left(F^{n}\right)^{D}: G_{r}\left[p^{n}\right]^{D} \rightarrow G_{r+n}\left[p^{n}\right]^{D}\right)$. Clearly $C_{n, r}=H_{n}\left(G_{r+n}\right)^{D}$. The isogeny $F: G_{r+1} \rightarrow G_{r}$ induces a morphism $C_{n, r+1} \rightarrow C_{n, r}$ which is generically an isomorphism. Over $\mathfrak{I}_{n, \infty, I}$ we have a universal morphism $\mathcal{O}_{F} / p^{n} \mathcal{O}_{F} \rightarrow H_{n}\left(G_{s}\right)^{D}$ for every $s \geq n-k$. The map $C_{n, s} \rightarrow G_{s}\left[p^{n}\right] / H_{n}\left(G_{s}\right) \simeq H_{n}\left(G_{s}\right)^{D}$ is generically an isomorphism as both group schemes are generically étale. Composing we then get an $\mathcal{O}_{F}$-equivariant map $\mathcal{O}_{F} / p^{n} \mathcal{O}_{F} \rightarrow C_{n, s}$ for every $s \geq n-k$. Using the morphisms $C_{n, r+1} \rightarrow C_{n, r}$, we get an $\mathcal{O}_{F}$-equivariant morphism $\mathcal{O}_{F} / p^{n} \mathcal{O}_{F} \rightarrow C_{n, r}$ for every $n$ which is generically an isomorphism. Passing to the projective limit, we get a $\mathcal{O}_{F}$-equivariant map $\mathcal{O}_{F} \otimes \mathbb{Z}_{p} \rightarrow \lim _{n} C_{n, r}$. Let $\mathrm{HT}^{u n}$ be the image of 1 in $\lim _{n} C_{n, r}$ via the Hodge-Tate map $\lim _{n} G_{r}\left[p^{n}\right] \rightarrow \omega_{G_{r}}$. Then HT ${ }^{u n}$ defines an $\left(\mathcal{O}_{F} \otimes \mathbb{Z}_{p}\right)^{*}$-equivariant map:

$$
\mathfrak{I G}_{\infty, \infty, I} \rightarrow \mathfrak{F}_{h, r, I}
$$

fitting in the commutative diagram:

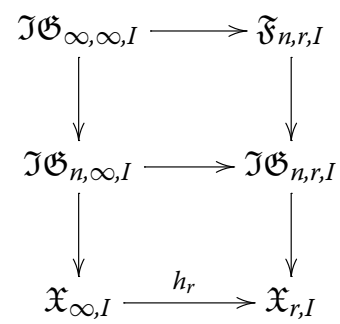

We then get an injective homomorphism $h_{r}^{*} \mathfrak{w}_{I} \rightarrow \mathfrak{w}_{I}^{\text {perf }}$. Moreover,

$$
\mathfrak{w}_{I}^{\text {perf }} \otimes h_{r}^{*} \mathfrak{w}_{I}^{-1} \subset\left(\mathcal{O}_{\mathfrak{I} \mathfrak{G}_{\infty, \infty, I}}\right)^{\left(\mathcal{O}_{F} \otimes \mathbb{Z}_{p}\right)^{*}}=\mathcal{O}_{\mathfrak{X} \infty, I}
$$

thanks to Corollary 5.4.

Corollary 6.2 We have a Tate trace map $\operatorname{Tr}_{r}: h_{r, *} \mathfrak{w}_{I}^{\text {perf }} \rightarrow \alpha^{-1} \mathfrak{w}_{I}$, which is functorial in $I$. 


\subsection{Descent along the anti-canonical tower}

Fix $\alpha \in \mathfrak{m} \backslash \mathfrak{m}^{2}$. Let $I=[1, \infty]$.

Lemma 6.3 The natural morphisms of sheaves

$$
\left.\mathcal{O}_{\mathfrak{X}_{r, \alpha, I}} \longrightarrow \lim _{k+1 \geq k^{\prime} \geq k \geq 0} \mathcal{O}_{\mathfrak{X}_{r, \alpha,}\left[p^{k}, p^{k^{\prime}}\right]} \text { and } \mathcal{O}_{\mathfrak{X}_{\infty, \alpha, I}}^{\longrightarrow} \lim _{k+1 \geq k^{\prime} \geq k \geq 0} \mathcal{O}_{\mathfrak{X}}{ }_{\infty, \alpha,\left[p^{k}, p^{k}\right.}\right]
$$

are isomorphisms.

Proof We prove the first statement. The second follows arguing as in [3, Lemme 6.6] using the Tate traces constructed in Proposition 5.2.

Let $U:=\operatorname{Spf} A$ be a formal open affine subscheme of $\overline{\mathfrak{M}}\left(\mu_{N}, \mathfrak{c}\right)$ over which $\omega_{G}$ is trivial. Let Ha be a lift of Ha. Arguing as in the proof of Lemma 3.7, one deduces that the inverse image of $U$ in $\mathfrak{X}_{r, \alpha, I}$ is Spf $R$ with $R:=A \otimes_{\mathbb{Z}_{p}} B_{\alpha}\langle u, w\rangle /\left(w \tilde{\mathrm{Ha}}^{r}-\alpha, \alpha u-p\right)$ and that for integers $1 \leq h<k$ the inverse image of $U$ in $\mathfrak{X}_{r, \alpha,\left[p^{h}, p^{k}\right]}$ is Spf $R_{h, k}$ with $R_{h, k}:=R\left\langle u_{h}, v_{k}\right\rangle /\left(\alpha^{p^{h}} u_{h}-p, u_{h} v_{k}-\alpha^{p^{k}-p^{h}}\right)$.

There are maps $R_{h, k} \rightarrow R_{h^{\prime}, k^{\prime}}$ for $h \leq h^{\prime} \leq k \leq k^{\prime}$ given by $u_{h} \mapsto \alpha^{p^{h^{\prime}}-p^{h}} u_{h^{\prime}}$ and $v_{k} \mapsto \alpha p^{k^{\prime}}-p^{k} v_{k^{\prime}}$. The argument in [3, Lemme 6.4] shows that the $\operatorname{map} R \rightarrow \lim _{k} R_{1, k}$ is an isomorphism. This proves the claim.

Theorem 6.4 The sheaf $\mathfrak{w}_{I}^{\text {perf }}$ descends to an invertible sheaf $\mathfrak{w}_{I}$ over $\mathfrak{X}_{r, \alpha, I}$ for $r \geq$ $\sup \left\{4,1+\log _{p} 2 g+1\right\}$ if $p \geq 3$ and $r \geq \sup \left\{6,1+\log _{p} 2 g+1\right\}$ if $p=2$. More pre-

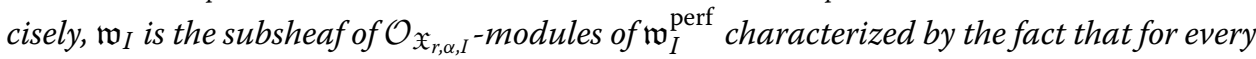
interval $J=\left[p^{k}, p^{k^{\prime}}\right]$ with $k^{\prime} \geq k \geq 0$ integers and denoting $\iota_{J, I}: \mathfrak{X}_{r, \alpha, J} \rightarrow \mathfrak{X}_{r, \alpha, I}$ the natural morphism, then $\iota_{J, I}^{*} \mathfrak{w}_{I}$ is the sheaf $\mathfrak{w}_{J}$ of Proposition 4.3 compatibly with the identification of Proposition 6.1.

Moreover, $\mathfrak{w}_{I}$ is free of rank 1 over every formal affine subscheme $U \subset \overline{\mathfrak{M}}\left(\mu_{N}, \mathfrak{c}\right)$ such that $\left.\omega_{G}\right|_{U}$ is trivial.

Proof The proof is analogous to the proof of [3, Thm. 6.4]. We set

$$
\mathfrak{w}_{I}:=\lim _{k+1 \geq k^{\prime} \geq k \geq 0} \mathfrak{w}_{\left[p^{k}, p^{k^{\prime}}\right]}
$$

where the limit is taken over integers $k, k^{\prime}$. Let $U:=\operatorname{Spf} A$ be a formal open affine subscheme of $\overline{\mathfrak{M}}\left(\mu_{N}, \mathfrak{c}\right)$ where $\omega_{G}$ is trivial. Let $W:=$ Spf $B$ be the inverse image of $U$ in $\mathfrak{X}_{r, \alpha, I}$. We prove that $\left.\mathfrak{w}_{I}\right|_{W}$ is a free $\mathcal{O}_{W}$-module of rank 1 and it descends $\left.\mathfrak{w}_{I}^{\text {perf }}\right|_{W}$.

We prove the claim for the minimal $r$ possible, i.e., $r=4$ if $p \geq 3$ and $r=6$ for if $p=2$. Let $\tilde{\mathrm{Ha}} r$ be a lift of the Hasse invariant over $U$. Thanks to Corollary 3.5 and Proposition 5.1, we can find elements $c_{n} \in \tilde{\mathrm{Ha}}_{r}{ }^{-\frac{p^{n}-1}{p-1}} \mathcal{O}_{\mathfrak{I} \mathfrak{G}_{n, r, \alpha},}(W)$ for integers $n \leq r$ and elements $c_{n} \in \tilde{\mathrm{Ha}}_{r}^{-p^{r}} \mathcal{O}_{\mathfrak{I} \mathfrak{G}_{n, \infty, \alpha, I}}(W)$ for general $r \leq n$ so that $c_{0}=1$ and $\operatorname{Tr}_{\mathfrak{I} \mathfrak{G}}\left(c_{n}\right)=c_{n-1}$. Define $b_{n}=\sum_{\sigma \in\left(\mathcal{O}_{F} / p^{n} \mathcal{O}_{F}\right)^{*}} \kappa(\tilde{\sigma}) \sigma\left(c_{n}\right)$ where $\tilde{\sigma}$ is a lift of $\sigma$ in $\left(\mathcal{O}_{F} \otimes \mathbb{Z}_{p}\right)^{*}$.

Using Lemma 2.6, we deduce that:

- The sequence $b_{n}$ converges to an element $b_{\infty}$,

- $b_{\infty}=1 \bmod \mathrm{Ha}^{-p^{r}} \alpha$, and in particular, $b_{\infty}$ generates $\mathfrak{w}_{I}^{\text {perf }}(W)$,

- $b_{\infty}=b_{r} \bmod \mathfrak{m}_{r-1} \tilde{\mathrm{Ha}}^{-p^{r}}$ (resp. $\mathfrak{m}_{r-2} \tilde{\mathrm{Ha}}^{-p^{r}}$ if $p=2$ ). 
Consider an interval $J=\left[p^{k}, p^{k+1}\right]$ and the commutative diagram:

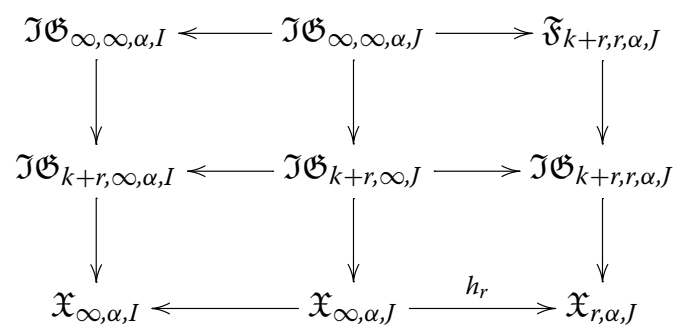

Let $T:=\operatorname{Spf} C$ be the inverse image of $W$ in $\mathfrak{X}_{r, \alpha, J}$. Then $\left.\mathfrak{w}_{J}\right|_{T}$ admits a generator $f$ as $C$-module constructed as follows. For every $0 \leq n \leq r+k$ take $c_{n}^{\prime} \in \tilde{H a}_{r}{ }^{\frac{p^{n}-1}{p-1}} \mathcal{O}_{\mathfrak{I} \mathfrak{G}_{n, r, \alpha}(}(T)$ such that $c_{0}^{\prime}=1, \operatorname{Tr}_{\mathfrak{I G}}\left(c_{n}^{\prime}\right)=c_{n-1}^{\prime}$ and $c_{n}^{\prime}=c_{n}$ if $n \leq r$. Take a section $s \in \mathcal{O}_{\mathfrak{F}_{k+r, r, j}}(T)$ which is $1 \bmod p^{2}$ and which generates $\mathfrak{w}_{r+k, r, J}(T)$ (see Lemma 4.5, and note that $1=k+r-(k+1)-2$ if $p \neq 2$ and $1=k+r-(k+1)-4$ if $p=2)$. Let $f:=\sum_{\sigma \in\left(\mathcal{O}_{F} / p^{k+r} \mathcal{O}_{F}\right)^{*}} \kappa(\tilde{\sigma}) \sigma\left(c_{r+k}^{\prime} s\right)$.

Then

$$
f=\sum_{\sigma \in\left(\mathcal{O}_{F} / p^{k+r} \mathcal{O}_{F}\right)^{*}} \kappa(\tilde{\sigma}) \sigma\left(c_{r+k}^{\prime}\right) \quad \bmod \mathrm{Ha}^{-\frac{p^{r+k}-1}{p-1} p^{2}}
$$

and it follows from 2.6 that

$$
\begin{aligned}
& \sum_{\sigma \in\left(\mathcal{O}_{F} / p^{k+r} \mathcal{O}_{F}\right)^{*}} \kappa(\tilde{\sigma}) \sigma\left(c_{r+k}^{\prime}\right) \\
= & \sum_{\sigma \in\left(\mathcal{O}_{F} / p^{r} \mathcal{O}_{F}\right)^{*}} \kappa(\tilde{\sigma}) \sigma\left(c_{r}\right) \bmod \left(\mathfrak{m}_{r-1} \tilde{\mathrm{Ha}}^{-\frac{p^{r}-1}{p-1}}, \ldots, \mathfrak{m}_{r+k-1} \tilde{\mathrm{Ha}}{ }^{-\frac{p^{r+k}-1}{p-1}}\right) \\
& \left(\operatorname{resp} . \bmod \left(\mathfrak{m}_{r-2} \tilde{\mathrm{Ha}}{ }^{-\frac{p^{r}-1}{p-1}}, \ldots, \mathfrak{m}_{r+k-2} \tilde{H a}^{-\frac{p^{r+k}-1}{p-1}}\right) \text { if } p=2\right) .
\end{aligned}
$$

Over the interval $\left[p^{k}, p^{k+1}\right]$, we have $p \in \alpha^{p^{k}} B_{\alpha, I}^{0}$, and it follows that

$$
\mathfrak{m}_{n} B_{\alpha, I}^{0} \subset\left(\alpha^{p^{n-1}}, \alpha^{p^{k}+p^{n-2}}, \ldots, \alpha^{(n-1) p^{k}+1}\right) .
$$

Moreover, $\alpha \in \tilde{\mathrm{Ha}}^{-p^{r+1}} T$. Assume $p \neq 2$. We claim that

$$
\left(\mathfrak{m}_{r-1} \tilde{\mathrm{Ha}}{ }^{-\frac{p^{r}-1}{p-1}}, \ldots, \mathfrak{m}_{r+k-1} \tilde{\mathrm{Ha}}{ }^{-\frac{p^{r+k}-1}{p-1}}\right) \subset\left(\alpha^{2}\right) .
$$

It is enough to check that:

$$
\left(\mathfrak{m}_{r-1} \alpha^{-1}, \mathfrak{m}_{r} \alpha^{-1}, \ldots, \mathfrak{m}_{r+k-1} \alpha^{-p^{k-1}}\right) \subset\left(\alpha^{2}\right)
$$

where the term $\mathfrak{m}_{r+k-1} \alpha^{-p^{k-1}}$ is missing if $k=0$.

This boils down to the set of inequalities:

- $1+2 \leq p^{r-2} ; 1+2 \leq p^{k}+p^{r-3} ; 1+2 \leq 2 p^{k}+p^{r-4}$.

- for $r \leq n \leq r+k-1$ :

$$
p^{n-r+1}+2 \leq p^{n-1} ; p^{n-r+1}+2 \leq p^{n-2}+p^{k} ; \ldots ; p^{n-r+1}+2 \leq(n-1) p^{k}+1 .
$$


When $p=2$, one proves similarly that

$$
\left(\mathfrak{m}_{r-2} \tilde{\mathrm{Ha}}^{-\frac{p^{r}-1}{p-1}}, \ldots, \mathfrak{m}_{r+k-2} \tilde{\mathrm{Ha}}^{-\frac{p^{r+k}-1}{p-1}}\right) \subset\left(\alpha^{2}\right) .
$$

It follows that

$$
f-b_{\infty} \in\left(\alpha^{2}, \tilde{\mathrm{Ha}}^{-\frac{p^{r+k}-1}{p-1}} p^{2}\right) \mathcal{O}_{\Im \mathfrak{G} \infty, \infty, \alpha, J}(T)
$$

so that $b_{\infty}=\left(1+\alpha^{2} u+\tilde{\mathrm{Ha}}{ }^{-\frac{p^{r+k}-1}{p-1}} p^{2} v\right) f$ for some elements $u, v \in \mathcal{O}_{\mathfrak{X}_{\infty, \alpha, J}}(T)$. Taking the Tate trace $\operatorname{Tr}_{r}: h_{r, *} \mathcal{O}_{\mathfrak{X}_{\infty, \alpha, J}} \rightarrow \alpha^{-1} \mathcal{O}_{\mathfrak{X}_{r, \alpha, J}}$ constructed in Proposition 5.2 we conclude that $\operatorname{Tr}_{r}\left(b_{\infty}\right)=f\left(1+\alpha^{2} \operatorname{Tr}_{r}(u)+\tilde{\mathrm{Ha}}{ }^{-\frac{p^{r+k}-1}{p-1}} p^{2} \operatorname{Tr}_{r}(v)\right)$. As $\operatorname{Tr}_{r}(u), \operatorname{Tr}_{r}(v) \in \alpha^{-1} \mathcal{O}_{\mathfrak{x}_{r, \alpha, J}}(T)$ by Proposition 5.2, we deduce that $\operatorname{Tr}_{r}\left(b_{\infty}\right)$ is a generator of $\left.\mathfrak{w}_{J}\right|_{T}$. Since the construction of $\operatorname{Tr}_{r}\left(b_{\infty}\right)$ is functorial in $J$ we get that $\mathfrak{w}_{I}(W)=\operatorname{Tr}_{r}\left(b_{\infty}\right) \cdot \lim _{k+1 \geq k^{\prime} \geq k \geq 0} \mathcal{O}_{\mathfrak{X}_{r, \alpha,\left[p^{k}, p^{k^{\prime}}\right]}}(W)=$ $\operatorname{Tr}_{r}\left(b_{\infty}\right) B$ thanks to Lemma 6.3. The theorem follows.

Proposition 6.5 The sheaves $\mathfrak{w}_{I}$ over each $\mathfrak{X}_{r, \alpha, I}$ glue to a sheaf still denoted $\mathfrak{w}_{I}$ over $\mathfrak{X}_{r, I}$. Let $\phi: \mathfrak{X}_{r+1, I} \rightarrow \mathfrak{X}_{r, I}$ be the Frobenius. We have an isomorphism

$$
\mathfrak{w}_{I} \simeq \phi^{*} \mathfrak{w}_{I}
$$

Proof Due to Theorem 6.4, the sheaf $\mathfrak{w}_{I}$ over $\mathfrak{X}_{r, \alpha, I}$ is canonically determined by the sheaves $\mathfrak{w}_{I}^{\text {perf }}$ and the sheaves $\mathfrak{w}_{J}$ for $J=\left[p^{k}, p^{k^{\prime}}\right]$. These glue for varying $\alpha$ and have compatible Frobenius morphisms by Sect. 4.3.2. The claim follows.

\subsection{Descent to the Iwasawa algebra}

Let $\mathfrak{Z}=\overline{\mathfrak{M}}\left(\mu_{N}, \mathfrak{c}\right) \times \mathfrak{W}_{F}^{0}$. For all $r \geq 0$, let $\mathfrak{Z}_{r}$ be the $\mathfrak{m}$-adic formal scheme representing the functor which associates with any m-adically complete $\Lambda_{F}^{0}$-algebra $R$ without $\Lambda_{F}^{0}$-torsion the equivalence classes of tuples $\left(h: \operatorname{Spf} R \rightarrow \overline{\mathfrak{M}}\left(\mu_{N}, \mathfrak{c}\right), \eta_{p}, \eta_{1}, \ldots, \eta_{g} \in\right.$ $\left.\mathrm{H}^{0}\left(\operatorname{Spf} R, h^{*} \operatorname{det} \omega_{G}^{(1-p) p^{r+1}}\right)\right)$ such that

$$
\mathrm{Ha}^{p^{r+1}} \eta_{p}=p \quad \bmod p^{2}, \quad \mathrm{Ha}^{p^{r+1}} \eta_{1}=T_{1} \quad \bmod p^{2}, \ldots, \mathrm{Ha}^{p^{r+1}} \eta_{g}=T_{g} \quad \bmod p^{2} .
$$

Two tuples $\left(h, \eta_{p}, \eta_{1}, \ldots, \eta_{g}\right)$ and $\left(h^{\prime}, \eta_{p}, \eta_{1}, \ldots, \eta_{g}\right)$ are declared equivalent if $h=h^{\prime}$ and

$$
\eta_{p}=\eta_{p}^{\prime}\left(1+p u_{p}\right), \quad \eta_{1}=\eta_{1}^{\prime}+\eta_{p} p u_{1}, \ldots, \eta_{g}=\eta_{g}^{\prime}+\eta_{p} p u_{g}
$$

for some $u_{p}, u_{1}, \ldots, u_{g} \in R$.

There is a cartesian diagram of formal schemes:

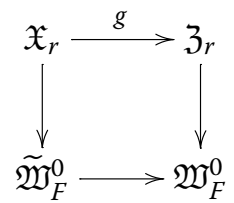

Theorem 6.6 (1) The natural map $\mathcal{O}_{\mathfrak{Z}_{r}} \rightarrow g_{\star} \mathcal{O}_{\mathfrak{X}_{r}}$ is an isomorphism.

(2) The sheaf $g_{\star} \mathfrak{w}_{I}$ (here $I=[1, \infty]$ ) is an invertible sheaf over $\mathfrak{Z}_{r}$ and $g^{\star} g_{\star} \mathfrak{w}_{I}=\mathfrak{w}_{I}$.

Proof Let $U:=\operatorname{Spf} A$ be a formal open affine subscheme of $\overline{\mathfrak{M}}\left(\mu_{N}, \mathfrak{c}\right)$ where $\omega_{G}$ is trivial. Let $W:=\operatorname{Spf} B$ be the inverse image of $U$ in $3 r$. In particular, we have elements $\eta_{p}, \eta_{1}, \ldots, \eta_{g}$ such that $B=A \llbracket T_{1}, \ldots, T_{g} \rrbracket\left\langle\eta_{p}, \eta_{1}, \ldots, \eta_{g}\right\rangle /\left(\mathrm{Ha}^{p^{r+1}} \eta_{p}-p, \mathrm{Ha}^{p^{r+1}} \eta_{1}-\right.$ $\left.T_{1}, \ldots, \mathrm{Ha}^{p^{r+1}} \eta_{g}-T_{g}\right)$. Arguing as in Lemma 3.7 we deduce that $B$ is normal. 
(1) The map $g$ is the base change of the morphism $\widetilde{\mathfrak{W}}_{F}^{0} \rightarrow \mathfrak{W}_{F}^{0}$ which is the m-adic completion of a blowup; therefore, it is proper. Thus $C:=g_{\star} \mathcal{O}_{\mathfrak{X}_{r}}(W)$ is a finite $B$-module. The map $B \rightarrow C$ is an isomorphism over the ordinary locus of $U$ and, hence, it is generically an isomorphism. As $\mathfrak{X}_{r}$ is also normal, it follows that $B=C$ as claimed.

(2) Let $Z:=g^{-1} W$. Thanks to (1) it suffices to prove that $\left.\mathfrak{w}_{I}\right|_{Z}$ is a free $\mathcal{O}_{Z}$-module of rank 1 . Let $Z^{\text {perf }}$ be the inverse image of $Z$ in $\mathfrak{X}_{\infty}$. We will actually prove that $\left.\mathfrak{w}_{I}^{\text {perf }}\right|_{Z \text { perf }}$

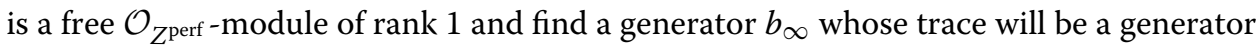
of $\left.\mathfrak{w}_{I}\right|_{Z}$.

We apply the construction of Sect. 3.3 with $A_{0}=\mathbb{Z}_{p}$. We thus obtain formal schemes $\mathfrak{Y}_{s}$ together with partial Igusa towers $\mathfrak{I} \mathfrak{G} \mathfrak{Y}_{n, s} \rightarrow \mathfrak{Y}_{s}$ for $n \leq s$.

Passing to the limit over Frobenius, we obtain $\mathfrak{I} \mathfrak{G Y} \mathfrak{Y}_{n, \infty} \rightarrow \mathfrak{Y}_{\infty}$. There is an obvious commutative diagram:

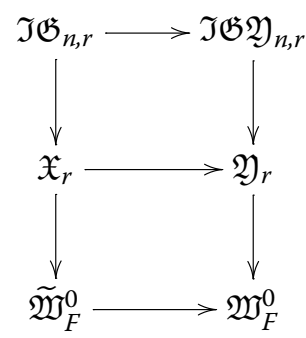

As in Corollary 3.5, one deduces from Proposition 3.4 the existence of elements

$$
c_{n}^{\prime} \in \tilde{\mathrm{Ha}}_{r}^{-\frac{p^{n}-1}{p-1}} \mathcal{O}_{\mathfrak{I} \mathfrak{G} \mathfrak{Y}_{n, r}}(W)
$$

for integers $n \leq r$ and elements $c_{n}^{\prime} \in \tilde{\mathrm{Ha}}_{r}^{-p^{r}} \mathcal{O}_{\mathfrak{I} \mathfrak{G} \mathfrak{Y}_{n, \infty}}(W)$ for general $r \leq n$ so that $c_{0}^{\prime}=1$ and $\operatorname{Tr}_{\mathfrak{I G}}\left(c_{n}^{\prime}\right)=c_{n-1}^{\prime}$. We call $c_{n}$ the pull back of $c_{n}^{\prime}$ in $\mathcal{O}_{\mathfrak{I} \mathfrak{G}_{n, \infty}}(Z)$. We can now repeat the proof of Theorem 6.4 using these elements $c_{n}$ to obtain a trivialization $b_{\infty}$ of $\left.\mathfrak{w}_{I}^{\text {perf }}\right|_{Z^{\text {perf }}}$ whose trace gives the trivialization of $\left.\mathfrak{w}_{I}\right|_{Z}$.

We set $\mathfrak{w}^{\kappa}=g_{\star} \mathfrak{w}_{I}$.

\subsection{The main theorem}

Let $H$ be the torsion subgroup of $\mathbb{T}\left(\mathbb{Z}_{p}\right)$. Let $\chi: H \rightarrow \Lambda_{F}^{\star}$ be the restriction of the universal character to $H$. Due to Lemma 2.4, it is a quotient of $\left(\mathcal{O}_{F} / p^{2} \mathcal{O}_{F}\right)^{*}$ and we view $\chi$ as a character of $\left(\mathcal{O}_{F} / p^{2} \mathcal{O}_{F}\right)^{*}$. For all $r \geq 0$, let

$$
\mathfrak{M}_{r}:=\mathfrak{Z}_{r} \times_{\mathfrak{W}_{F}^{0}} \mathfrak{W}_{F}
$$

Let $\mathcal{M}_{r}$ be the analytic adic space associated with $\mathfrak{M}_{r}$. In other words, $\mathcal{M}_{r}$ is the open subset of the $\bar{M}\left(\mu_{N}, \mathfrak{c}\right) \times_{\text {Spec } \mathbb{Z}_{p}} \mathcal{W}_{F}$ defined by the conditions:

$$
\left|\tilde{\mathrm{Ha}}^{p^{r+1}}\right| \geq \sup _{\alpha \in \mathfrak{m}}\{|\alpha|\},
$$

where $\tilde{H a}$ is a local lift of the Hasse invariant. Over $\mathcal{M}_{r}$ we have a canonical subgroup $C_{2}$ of level 2. Let $\mathcal{I G M}_{2, r}$ be the torsor of trivializations of $C_{2}^{D}$. Let $\mathfrak{I} \mathfrak{G M} \mathfrak{M}_{2, r} \stackrel{h}{\rightarrow} \mathfrak{M}_{r}$ be the normalization. It carries an action of $\left(\mathcal{O}_{F} / p^{2} \mathcal{O}_{F}\right)^{*}$.

Let $\mathfrak{w}^{\chi}$ be the subsheaf of $\left(h_{2}\right)_{\star} \mathcal{O}_{\mathfrak{I} \mathfrak{G} \mathfrak{M}_{2, r}}$ where $\left(\mathcal{O}_{F} / p^{2} \mathcal{O}_{F}\right)^{*}$ acts via the character $\chi^{-1}: H \rightarrow \Lambda_{F}^{*}$ composed with the projection $\left(\mathcal{O}_{F} / p^{2} \mathcal{O}_{F}\right)^{*} \rightarrow H$. This is a coherent sheaf, invertible over the ordinary locus and over the analytic fiber $\mathcal{M}_{r}$. 
We define $\mathfrak{w}^{\kappa^{\text {un }}}:=\left(s^{\star} \mathfrak{w}^{\kappa}\right) \otimes \mathfrak{w}^{\chi}$ where $s: \mathfrak{M}_{r} \rightarrow \mathfrak{Z}_{r}$ is the projection. We let $\omega^{\kappa^{\text {un }}}$ be the associated sheaf over $\mathcal{M}_{r}$.

Theorem 6.7 The sheaf $\omega^{\kappa^{\mathrm{un}}}$ over $\mathcal{M}_{r}$ enjoys the following properties:

1. the restriction of $\omega^{\kappa}$ un to the classical analytic space $\mathcal{M}_{r} \times \operatorname{Spa}\left(\mathbb{Z}_{p}, \mathbb{Z}_{p}\right) \operatorname{Spa}\left(\mathbb{Q}_{p}, \mathbb{Z}_{p}\right)$ is the sheaf defined in [2, Def. 3.6];

2. for all locally algebraic weight $k \chi: \mathbb{T}\left(\mathbb{Z}_{p}\right) \rightarrow \mathcal{O}_{\mathbb{C}_{p}}^{*}$ where $k$ is an algebraic weight and $\chi$ is a finite character, then $\left.\omega^{\kappa^{\mathrm{un}}}\right|_{k \chi}=\omega^{k}(\chi)$ is the sheaf of weight $k$ modular forms and nebentypus $\chi$.

3. If $i: \mathcal{M}_{r+1} \rightarrow \mathcal{M}_{r}$ is the inclusion and $\phi: \mathcal{M}_{r+1} \rightarrow \mathcal{M}_{r}$ is the Frobenius, then we have a canonical isomorphism $i^{\star} \omega^{\kappa^{\text {un }}} \simeq \phi^{\star} \omega^{\kappa^{\text {un }}}$.

\section{Overconvergent forms in characteristic $\boldsymbol{p}$}

Specializing the sheaf $\omega^{\kappa^{\text {un }}}$ of Theorem 6.7 to characteristic $p$ points of $\mathcal{W}_{F}$, we obtain sheaves of overconvergent Hilbert modular forms in characteristic $p$. The goal of this section is to describe them via a construction purely in characteristic $p$.

\subsection{The characteristic $p$ Igusa tower}

Let $\bar{M}\left(\mu_{N}, \mathfrak{c}\right)_{\mathbb{F}_{p}}$ be the special fiber of $\overline{\mathfrak{M}}\left(\mu_{N}, \mathfrak{c}\right)$ and denote by $\bar{M}_{\text {ord }}\left(\mu_{N}, \mathfrak{c}\right)_{\mathbb{F}_{p}}$ the ordinary locus. It is an open dense subscheme of $\bar{M}\left(\mu_{N}, \mathfrak{c}\right)_{\mathbb{F}_{p}}$, smooth over Spec $\mathbb{F}_{p}$. Fix a positive integer $n$. Over $\bar{M}\left(\mu_{N}, \mathfrak{c}\right)_{\mathbb{F}_{p}}$ we have a canonical subgroup of level $n$, denoted by $H_{n}$. It is the kernel of the $n$th power Frobenius map $F^{n}: G \rightarrow G^{\left(p^{n}\right)}$. The canonical subgroup over $\bar{M}_{\text {ord }}\left(\mu_{N}, \mathfrak{c}\right)_{\mathbb{F}_{p}}$ is of multiplicative type and its dual $H_{n}^{D}$ is étale locally isomorphic to $\mathcal{O}_{F} / p^{n} \mathcal{O}_{F}$. We denote by $\overline{\mathrm{IG}}_{n \text {,ord }} \rightarrow \bar{M}_{\text {ord }}\left(\mu_{N}, \mathfrak{c}\right)_{\mathbb{F}_{p}}$ the finite, étale and Galois cover for the group $\left(\mathcal{O}_{F} / p^{n} \mathcal{O}_{F}\right)^{*}$ of trivializations of $H_{n}^{D}$. Passing to the projective limit over $n$, we get a scheme $\overline{\mathrm{IG}}_{\infty \text {,ord }}$ over $\bar{M}_{\text {ord }}\left(\mu_{N}, \mathfrak{c}\right)_{\mathbb{F}_{p}}$. For every $n$ define $\overline{\mathrm{IG}}_{n} \rightarrow \bar{M}\left(\mu_{N}, \mathfrak{c}\right)_{\mathbb{F}_{p}}$ to be the normalization of $\bar{M}\left(\mu_{N}, \mathfrak{c}\right)_{\mathbb{F}_{p}}$ in $\overline{\mathrm{IG}}_{n \text {,ord }}$. The scheme $\overline{\mathrm{IG}}_{n}$ is finite over $\bar{M}\left(\mu_{N}, \mathfrak{c}\right)_{\mathbb{F}_{p}}$ and carries an action of the group $\left(\mathcal{O}_{F} / p^{n} \mathcal{O}_{F}\right)^{*}$. It is characterized by the following universal property:

Lemma 7.1 For every normal $\mathbb{F}_{p}$-algebra $R$ and every $R$-valued point $x \in \bar{M}\left(\mu_{N}, \mathfrak{c}\right)(R)$ such that the ordinary locus (Spec R) ord is dense in Spec $R$, the R-valued points of $\overline{\mathrm{IG}}_{n}$ over $x$ consist of the $\mathcal{O}_{F}$-equivariant morphisms $\mathcal{O}_{F} / p^{n} \mathcal{O}_{F} \rightarrow H_{n, x}^{D}$, which are isomorphisms over $(\text { Spec } R)_{\text {ord. Here }} H_{n, x}$ is the pull back of the canonical subgroup to Spec $R$ via $x$.

Let $h_{n+1}: \overline{\mathrm{IG}}_{n+1} \rightarrow \overline{\mathrm{IG}}_{n}$ (with the convention $\overline{\mathrm{IG}}_{0}=\bar{M}\left(\mu_{N}, \mathfrak{c}\right)_{\mathbb{F}_{p}}$ ). Let us denote by

$$
\operatorname{Tr}_{\mathrm{IG}}:\left(h_{n+1}\right)_{\star} \mathcal{O}_{\overline{\mathrm{IG}}_{n+1}} \rightarrow \mathcal{O}_{\overline{\mathrm{IG}}_{n}}
$$

the trace of this morphism.

Lemma 7.2 For all $n \geq 0$, we have $\mathrm{Hdg}^{p^{n}} \subset \operatorname{Tr}_{\mathrm{IG}}\left(\left(h_{n+1}\right)_{\star} \mathcal{O}_{\overline{\mathrm{IG}}_{n}}\right)$.

Proof Similar to the proof of Proposition 3.4.

Corollary 7.3 Let Spec $A$ be an open subset of $\bar{M}\left(\mu_{N}, \mathfrak{c}\right)_{\mathbb{F}_{p}}$ such that the sheaf Hdg is trivial. Let us identify $\mathrm{Ha}$ with a generator of $\mathrm{Hdg}$. There is a sequence of elements $c_{0}=1$, $c_{n} \in \mathrm{Ha}^{-\frac{p^{n}-1}{p-1}} \mathcal{O}_{\overline{\mathrm{IG}}_{n}}(\operatorname{Spec} A)$ for $n \geq 1$ such that $\operatorname{Tr}_{\mathrm{IG}}\left(c_{n}\right)=c_{n-1}$. 


\subsection{Formal schemes attached to the Hilbert modular variety in characteristic $p$}

Let $\overline{\mathfrak{m}}$ be the maximal ideal $\left(T_{1}, \ldots, T_{g}\right)$ of $\Lambda_{F}^{0} / p \Lambda_{F}^{0}$. We set $\mathfrak{W}_{F,\{\infty\}}^{0}=\operatorname{Spf} \Lambda_{F}^{0} / p \Lambda_{F}^{0}$. Recall that $\widetilde{\mathfrak{W}}_{F,\{\infty\}}^{0}$ is the blowup of $\mathfrak{W}_{F,\{\infty\}}^{0}$ along $\overline{\mathfrak{m}}$.

In Sect. 6.3, we define an m-adic formal scheme $\mathfrak{Z}_{r} \rightarrow \mathfrak{W}_{F}^{0}$. We set $\mathfrak{Z}_{r,\{\infty\}}=\mathfrak{Z}_{r} \times_{\mathfrak{W}_{F}^{0}}$ $\mathfrak{W}_{F,\{\infty\}}^{0}$. Its ordinary locus is $\mathfrak{Z}_{\text {ord },\{\infty\}}=\bar{M}_{\text {ord }}\left(\mu_{N}, \mathfrak{c}\right)_{\mathbb{F}_{p}} \times$ Spec $\mathbb{F}_{p}$ Spf $\mathfrak{W}_{F,\{\infty\}}^{0}$.

In Sect. 3.4, we define a formal scheme $\mathfrak{X}_{r,\{\infty\}}$ over $\widetilde{\mathfrak{W}}_{F,\{\infty\}}^{0}$ and it follows from the definitions that:

$$
\mathfrak{X}_{r,\{\infty\}}=\mathfrak{Z}_{r,\{\infty\}} \times_{\mathfrak{W}_{F,\{\infty\}}^{0}} \widetilde{\mathfrak{W}}_{F,\{\infty\}}^{0} .
$$

Let $\mathfrak{I} \mathfrak{G} \mathfrak{Z}_{n, r,\{\infty\}}=\overline{\mathrm{IG}}_{n} \times{ }_{\text {Spec } \mathbb{F}_{p}} \mathfrak{Z}_{r,\{\infty\}}$ be the partial Igusa tower of level $n$ over $\mathfrak{Z}_{r,\{\infty\}}$. Passing to the limit over $n$, we get an $\overline{\mathfrak{m}}$-adic formal scheme $h: \mathfrak{I} \mathfrak{G} \mathfrak{Z}_{\infty, r,\{\infty\}} \rightarrow \mathfrak{Z}_{r,\{\infty\}}$ which carries an action of $\mathbb{T}\left(\mathbb{Z}_{p}\right)$.

Lemma 7.4 1. The formal scheme $\mathfrak{I} \mathfrak{G} \mathfrak{Z}_{n, r,\{\infty\}}$ is normal.

2. We have $\mathfrak{I G}_{n, r,\{\infty\}}=\mathfrak{I} \mathfrak{G} \mathfrak{Z}_{n, r,\{\infty\}} \times \mathfrak{Z}_{r,\{\infty\}} \mathfrak{X}_{r,\{\infty\}}$, where $\mathfrak{I G}_{n, r,\{\infty\}}$ is defined in Sect. 3.4.

3. $\left(h_{\star} \mathcal{O}_{\mathfrak{I} \mathfrak{G} \mathfrak{Z}_{\infty, r,\{\infty\}}}\right)^{\mathbb{T}\left(\mathbb{Z}_{p}\right)}=\mathcal{O}_{\mathfrak{Z}_{r,\{\infty\}}}$.

Proof Easy and left to the reader.

We have the following:

Proposition 7.5 For any normal, $\overline{\mathfrak{m}}$-adically complete torsion free $\Lambda_{F}^{0} / p \Lambda_{F}^{0}$-algebra, the $R$-valued points $\mathfrak{I G} \mathfrak{Z}_{n, r, \infty}(R)$ classify tuples $\left(x, \eta_{T_{1}}, \ldots, \eta_{T_{g}}, \psi_{n}\right)$ where

- $x \in \bar{M}\left(\mu_{N}, \mathfrak{c}\right)(R)$,

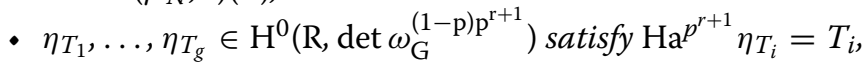

- $\psi_{n}: \mathcal{O}_{F} / p^{n} \mathcal{O}_{F} \rightarrow H_{n}^{D}$ is a $\mathcal{O}_{F}$-linear morphism of group schemes which is an isomorphism over $(\text { Spec } R)_{\text {ord }}$.

\subsection{Convergent Hilbert modular forms in characteristic $p$}

Let $\bar{\kappa}:\left(\mathcal{O}_{F} \otimes \mathbb{Z}_{p}\right)^{*} \rightarrow\left(\Lambda_{F}^{0} / p \Lambda_{F}^{0}\right)^{*}$ be the reduction modulo $p$ of the character $\kappa$. Following Katz [14], we define

$$
\mathfrak{w}_{\{\infty\}}:=\mathcal{O}_{\mathfrak{I} \mathfrak{G} \mathfrak{Z}_{\infty, \text { ord },\{\infty\}}}\left[\overline{\mathcal{K}}^{-1}\right] .
$$

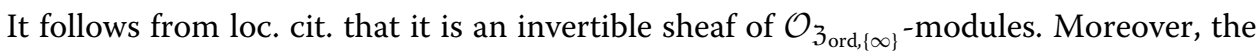
Frobenius on $\mathfrak{I} \mathfrak{G} \mathfrak{Z}_{\infty, \text { ord },\{\infty\}}$ defines an isomorphism $\phi^{*} \mathfrak{w}_{\{\infty\}} \simeq \mathfrak{w}_{\{\infty\}}$.

\subsection{Overconvergent Hilbert modular forms in characteristic $p$}

Let $h: \mathfrak{I} \mathfrak{G} \mathfrak{Z}_{\infty, r,\{\infty\}} \rightarrow \mathfrak{Z}_{r,\{\infty\}}$ be the structural morphism. As in the previous section, we define the subsheaf $\mathfrak{w}_{\{\infty\}}:=h_{*} \mathcal{O}_{\mathfrak{I G} \mathfrak{Z}_{\infty, r,\{\infty\}}}\left[\bar{\kappa}^{-1}\right]$ of $h_{*} \mathcal{O}_{\mathfrak{I G} \infty, r,\{\infty\}}$. It is a sheaf of $\mathcal{O}_{\mathfrak{Z}_{r,\{\infty\}}}$ modules. Our main theorem is

Theorem 7.6 Assume that $r \geq 2$ (resp. $r \geq 3$ ifp = 2). Then the sheaf $\mathfrak{w}_{\{\infty\}}$ is an invertible

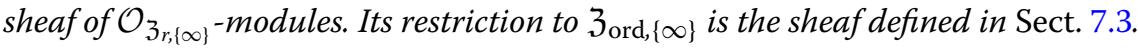

Proof The proof is local on $\bar{M}\left(\mu_{N}, \mathfrak{c}\right)_{\mathbb{F}_{p}}$. Let Spec $A$ be an open subset of $\bar{M}\left(\mu_{N}, \mathfrak{c}\right)_{\mathbb{F}_{p}}$ where the Hodge ideal is trivial. We denote abusively Ha a generator. Let $\operatorname{Spf} R$ be the inverse image of Spec $A$ in $\mathfrak{Z}_{r,\{\infty\}}$, Spf $R_{n}$ the inverse image in $\mathfrak{I} \mathfrak{G} \mathfrak{U}_{n, r,\{\infty\}}$ and $\operatorname{Spf} R_{\infty}$ the inverse image in $\mathfrak{I} \mathfrak{G} \mathfrak{Z}_{\infty, r,\{\infty\}}$. By Lemma 7.4, $R_{\infty}^{\left(\mathcal{O}_{F} \otimes \mathbb{Z}_{p}\right)^{*}}=R$. Thus to prove the theorem, it suffices 
to show that there exists an invertible element $x \in R_{\infty}$ such that $\sigma(x)=\bar{\kappa}^{-1}(\sigma) x$ for every $\sigma \in\left(\mathcal{O}_{F} \otimes \mathbb{Z}_{p}\right)^{*}$.

By Corollary 7.3, there exist elements $c_{n} \in \mathrm{Ha}^{-\frac{p^{n}-1}{p-1}} R_{n}$ such that $\operatorname{Tr}_{R_{n} / R_{n-1}}\left(c_{n}\right)=c_{n-1}$ and $c_{0}=1$. Define $b_{n}:=\sum_{\sigma \in G_{n}} \bar{\kappa}(\tilde{\sigma}) \sigma\left(c_{n}\right) \in \mathrm{Ha}^{-\frac{p^{n}-1}{p-1}-p} R_{n}$ for $n \geq 1$. Here $\tilde{\sigma} \in\left(\mathcal{O}_{F} \otimes\right.$ $\left.\mathbb{Z}_{p}\right)^{*}$ is a lift of $\sigma \in\left(\mathcal{O}_{F} / p^{n} \mathcal{O}_{F}\right)^{*}$. By Lemma 2.6, we deduce that

- $b_{n}-b_{n-1} \in\left(T_{1}^{p^{n-1}}, \ldots, T_{g}^{p^{n-1}}\right) \mathrm{Ha}^{-\frac{p^{n}-1}{p-1}} R_{n}$ if $n \geq 1$ and $p \geq 3$,

- $b_{n}-b_{n-1} \in\left(T_{1}^{p^{n-2}}, \ldots, T_{g}^{p^{n-2}}\right) \mathrm{Ha}^{-\frac{p^{n}-1}{p-1}} R_{n}$ if $n \geq 2$ and $p=2$,

- $b_{1}-1 \in\left(T_{1}, \ldots, T_{g}\right) \mathrm{Ha}^{-1} R_{1}$ for all $p$.

One then concludes that $\left\{b_{n}\right\}_{n}$ is a Cauchy sequence of elements of $R_{\infty}$ converging to a unit $b_{\infty}:=\lim _{n} b_{n}$ of $R_{\infty}$ having the property that $\sigma\left(b_{\infty}\right)=\bar{\kappa}^{-1}(\sigma) b_{\infty}$ for every $\sigma \in\left(\mathcal{O}_{F} \otimes \mathbb{Z}_{p}\right)^{*}$.

\subsection{Comparison with the sheaf $\mathfrak{w}_{[1, \infty]}$}

In this section, we work over $\mathfrak{X}_{r,\{\infty\}}$ and prove that the specialization at $\{\infty\}$ of the sheaf $\mathfrak{w}_{[1, \infty]}$ of Theorem 6.4 equals the pull back to $\mathfrak{X}_{r,\{\infty\}}$ of the sheaf $\mathfrak{w}_{\{\infty\}}$ defined in Sect. 7.4.

Proposition 7.7 Let $\alpha \in \mathfrak{m}$. For every integer $k_{0} \geq 1$, the obvious inclusion $\mathfrak{w}_{\left[p^{\left.k_{0}, \infty\right]}\right.} \subset$ $\mathcal{O}_{\Im \mathfrak{G}}{ }_{\infty, \infty, \alpha,\left[p^{\left.k_{0}, \infty\right]}\right.}$ factors modulo $\alpha^{p^{k_{0}}-p^{k_{0}-1}-1}$ as a morphism

$$
\mathfrak{w}_{\left[p^{\left.k_{0}, \infty\right]}\right.} \rightarrow \mathcal{O}_{\mathfrak{I G}_{r+k_{0}, r, \alpha,\left[p^{k_{0}, \infty}\right]}} / \alpha^{p^{k_{0}}-p^{k_{0}-1}-1} .
$$

The restriction of $\mathfrak{w}_{I}$ to $\mathfrak{X}_{r, \alpha,\{\infty\}}$ is a subsheaf of $\mathcal{O}_{\mathfrak{I} \mathfrak{I}_{\infty, r, \alpha,\{\infty\}}}$ which identifies canonically to $\mathfrak{w}_{\{\infty\}}$

Proof Fix an integer $k_{0} \geq 1$. Let $\operatorname{Spf} B$ be an open affine of $\mathfrak{X}_{r, \alpha,\left[p^{\left.k_{0}, \infty\right]}\right.}$. Assume that $\operatorname{Hdg}$ is trivial on $\operatorname{Spf} B$, generated by Ha. Fix elements $c_{0}=1$ and, for $1 \leq n \leq k_{0}+r$, $c_{n} \in \mathrm{Ha}{ }^{-\frac{p^{n}-1}{p-1}} \mathcal{O}_{\mathfrak{I} \mathfrak{G}_{n, r, \alpha,\left[p^{k}, \infty\right]}}(\operatorname{Spf} B)$ such that $\operatorname{Tr}_{\mathfrak{I} \mathfrak{G}}\left(c_{n}\right)=c_{n-1}$. Complete the sequence by choosing, for $n \geq r+k_{0}+1$, elements $c_{n} \in \tilde{\mathrm{Ha}}{ }^{-p^{r+k_{0}}} \mathcal{O}_{\mathfrak{I} \mathfrak{G}_{n, \infty, \alpha,[} k_{0, \infty]}}$ (Spf $\left.B\right)$ satisfying $\operatorname{Tr}_{\mathfrak{I G}}\left(c_{n}\right)=c_{n-1}$. Set $b_{n}=\sum_{\sigma \in\left(\mathcal{O}_{F} / p^{n} \mathcal{O}_{F}\right)^{*}} \kappa(\tilde{\sigma}) \sigma . c_{n}$, where $\tilde{\sigma}$ is a lift of $\sigma$ in $\mathbb{T}\left(\mathbb{Z}_{p}\right)$. The sequence $b_{n}$ converges in $\mathcal{O}_{\mathfrak{I} \mathfrak{c}}{ }_{\infty, \infty,[p}^{\left.k_{0}, \infty\right]}$ to a generator $b_{\infty}$ of the sheaf $\mathfrak{w}_{I}^{\text {perf }}$. By Lemma 2.6, for all $n \geq r+k_{0}, b_{n}=b_{r+k_{0}} \bmod \mathfrak{m}_{r+k_{0}-1} \tilde{\mathrm{Ha}}^{-p^{r+k_{0}}}\left(\right.$ resp. $\bmod \mathfrak{m}_{r+k_{0}-2} \tilde{\mathrm{Ha}}^{-p^{r+k_{0}}}$ if $p=2)$. It follows that $b_{\infty}=b_{r+k_{0}} \bmod \alpha^{p^{k_{0}}-p^{k_{0}-1}}$.

Fix now an interval $\left[p^{k}, p^{k+1}\right]$ with $k \geq k_{0}$. Let $\operatorname{Spf} C$ be the inverse image of $\operatorname{Spf} B$ in $\mathfrak{X}_{r, \alpha,\left[p^{k}, p^{k+1}\right]}$. Fix elements $c_{n}^{\prime} \in \tilde{H}^{-\frac{p^{n}-1}{p-1}} \mathcal{O}_{\mathfrak{I G}_{n, r \alpha,\left[p^{k}, p^{k+1}\right]}}(\operatorname{Spf} C)$ for $r+k_{0}+1 \leq n \leq r+k$ satisfying $\operatorname{Tr}_{\mathfrak{I G}}\left(c_{n}^{\prime}\right)=c_{n-1}^{\prime}$ for $n \geq r+k_{0}+2$ and $\operatorname{Tr}_{\mathfrak{I G}}\left(c_{r+k_{0}+1}^{\prime}\right)=c_{r+k_{0}}$. There is a generator $f$ of the sheaf $\mathfrak{w}_{I}$ over Spf $C$ such that $f=\sum_{\sigma \in\left(\mathcal{O} / p^{r+k} \mathcal{O}\right)^{*}} \kappa(\tilde{\sigma}) \sigma \cdot c_{r+k}^{\prime}$ $\bmod p \tilde{\mathrm{Ha}}^{-p^{r+k}}$. As in $C$ we have $\alpha^{p^{k}} \mid p$ and $\tilde{\mathrm{Ha}}^{-p^{r+k}} \mid \alpha^{p^{k-1}}$ it follows that $f=$ $\sum_{\sigma \in\left(\mathcal{O} / p^{r+k} \mathcal{O}\right)^{*}} \kappa(\tilde{\sigma}) \sigma \cdot c_{r+k}^{\prime} \bmod \alpha^{p^{k_{0}}-p^{k_{0}-1}}$. Using Lemma 2.6 one more time, we deduce that $f=b_{r+k_{0}} \bmod \alpha^{p^{k_{0}}-p^{k_{0}-1}}$. As a consequence, in $\mathcal{O}_{\mathfrak{I G}}{ }_{\infty, \infty, \alpha,\left[p^{k, p} k^{k+1}\right]}(\operatorname{Spf} C)$ we have $f=b_{\infty}=b_{r+k_{0}} \bmod \alpha^{p^{k_{0}}-p^{k_{0}-1}}$. Using Tate's traces, we get $f=\operatorname{Tr}_{r}\left(b_{\infty}\right)=b_{\infty}$ $\bmod \alpha^{p^{k_{0}}-p^{k_{0}-1}-1} \mathfrak{w}_{\left[p^{k}, p^{k+1}\right]}^{\text {perf }}(\operatorname{Spf} C)$. As this relation holds on all intervals $\left[p^{k}, p^{k+1}\right]$, it fol- 
lows that $\operatorname{Tr}_{r}\left(b_{\infty}\right)=b_{\infty} \bmod \alpha^{p^{k_{0}}-p^{k_{0}-1}-1} \mathfrak{w}_{\left[p^{\left.k_{0}, \infty\right]}\right.}^{\text {perf }}(\operatorname{Spf} B)$. As a result, $\operatorname{Tr}_{r}\left(b_{\infty}\right)=b_{r+k_{0}}$ $\bmod \alpha^{p^{k_{0}}-p^{k_{0}-1}-1} \mathcal{O}_{\mathfrak{I G}}{ }_{\infty, \infty, \alpha,\left[p^{\left.k_{0}, \infty\right]}\right.}(\operatorname{Spf} B)$.

It follows that there is a morphism $\mathfrak{w}_{\left[p^{\left.k_{0}, \infty\right]}\right.} \rightarrow \mathcal{O}_{\Im G_{r+k_{0}, r, \alpha,\left[p^{k}, \infty\right]}} / \alpha^{p^{k_{0}}-p^{k_{0}-1}-1}$ which on Spf $B$ sends $\operatorname{Tr}_{r}\left(b_{\infty}\right)$ on $b_{r+k_{0}}$. It factors the map $\mathfrak{w}_{\left[p^{\left.k_{0}, \infty\right]}\right.} \rightarrow \mathcal{O}_{\mathfrak{I} G}{ }_{\infty, \infty, \alpha,[p} k_{0, \infty]} / \alpha^{p^{k}}-p^{k_{0}-1}-1$. Comparing the definition of $b_{r+k_{0}}$ and the construction of the sheaf $\mathfrak{w}_{\{\infty\}}$, we obtain that the restriction of $\mathfrak{w}_{[1, \infty]}$ to $\mathfrak{X}_{r,\{\infty\}}$ is $\mathfrak{w}_{\{\infty\}}$.

\subsection{Analytic overconvergent Hilbert modular forms in characteristic $p$}

We now let $\mathcal{M}_{r,\{\infty\}}=\mathcal{M}_{r} \times \mathcal{W}_{F} \mathcal{W}_{F,\{\infty\}}$. Concretely, $\mathcal{M}_{r,\{\infty\}}$ is the open subset of $\bar{M}\left(\mu_{N}, \mathfrak{c}\right) \times_{\operatorname{Spec}_{p}} \mathcal{W}_{F,\{\infty\}}$ where

$$
\left|\mathrm{Ha}^{p^{r+1}}\right| \geq \sup _{\alpha \in \overline{\mathfrak{m}}}|\alpha|
$$

Let us recall that we denoted by $\bar{\kappa}^{\text {un }}: \mathbb{T}\left(\mathbb{Z}_{p}\right) \rightarrow\left(\Lambda_{F} / p \Lambda_{F}\right)^{*}$ the universal $\bmod p$ character.

Let $\omega^{\bar{\kappa}^{\text {un }}}$ be the pull back of $\omega^{\kappa^{\text {un }}}$ to $\mathcal{M}_{r,\{\infty\}}$. An $r$-overconvergent Hilbert modular form of weight $\bar{\kappa}^{\text {un }}$ is a global section of $\omega^{\bar{\kappa}^{\text {un }}}$. Here is the desired, à la Katz, description.

Proposition 7.8 An r-overconvergent modular form $f$ of weight $\bar{\kappa}^{\text {un }}$ is a functorial rule which associates with a tuple $\left(\left(R, R^{+}\right), x: \operatorname{Spa}\left(R, R^{+}\right) \rightarrow \mathcal{M}_{r,\{\infty\}}, \psi: \mathcal{O}_{F} \otimes \mathbb{Z}_{p} \simeq\right.$ $\left.\lim _{n} x^{*} H_{n}^{D}\right)$ an element in $f(x, \psi) \in R$, where:

1. $\left(R, R^{+}\right)$is a complete affinoid Tate algebra,

2. $x^{*} H_{n}^{D}$ is the pullback of the dual canonical subgroup of level $n$ to $\operatorname{Spa}\left(R, R^{+}\right)$,

3. The isomorphism $\psi$ is $\mathbb{T}\left(\mathbb{Z}_{p}\right)$-equivariant,

4. For all $\sigma \in \mathbb{T}\left(\mathbb{Z}_{p}\right), f(x, \psi \circ \sigma)=\left(\bar{\kappa}^{\mathrm{un}}\right)^{-1}(\sigma) f(x, \psi)$.

5. There exists a rational cover $\operatorname{Spa}\left(R, R^{+}\right)=\cup \operatorname{Spa}\left(R_{i}, R_{i}^{+}\right)$and for each $i$ a bounded and open subring $R_{i, 0} \subset R_{i}^{+}$such that $\left.x^{\star} G\right|_{S p a\left(R_{i}, R_{i}^{+}\right)}$comes from a semi-abelian scheme $G_{0}$ over Spf $R_{i, 0}$ and the isomorphism $\left.\psi\right|_{S p a\left(R_{i}, R_{i}^{+}\right)}$comes from a group scheme morphism $\psi_{0}: \mathcal{O}_{F} \otimes \mathbb{Z}_{p} \rightarrow \lim _{n}\left(G_{0}\left[F^{n}\right]\right)^{D}$ defined over SpfR $R_{i, 0}$ (where $\left[F^{n}\right]$ means the kernel of the nth power of the Frobenius isogeny).

Proof Take $\left(R, R^{+}\right)$as in the proposition. Without loss of generality, we can assume that $R$ has a noetherian ring of definition. Using (5), we observe that the rule $f$ defines compatible sections of $\mathrm{H}^{0}\left(\operatorname{Spf} R_{i, 0}, \mathfrak{w}_{\{\infty\}}\right) \otimes_{R_{i, 0}} R$ which glue, by the sheaf property, to a section of $\omega^{\bar{\kappa}^{u n}}$ on $\operatorname{Spa}\left(R, R^{+}\right)$.

\section{Overconvergent arithmetic Hilbert modular forms}

\subsection{The Shimura variety $\bar{M}\left(\mu_{N}, \mathfrak{c}\right)_{G}$}

Consider the group $G:=\operatorname{Res}_{F / \mathbb{Q}} \mathrm{GL}_{2}$ and $G^{*}:=G \times \operatorname{Res}_{F / \mathbb{Q}} \mathbb{G}_{m} \mathbb{G}_{m}$, where the morphism $G \longrightarrow \operatorname{Res}_{F / \mathbb{Q}} \mathbb{G}_{m}$ is the determinant. So far we have worked on the Shimura variety associated with the group $G^{*}$. From the point of view of automorphic forms, it is useful to work with the Shimura variety defined by the group $G$.

Let $\mathcal{O}_{F}^{+, *}$ be the group of totally real units of $\mathcal{O}_{F}$ and let $U_{N} \subset \mathcal{O}_{F}^{*}$ be the group of units congruent to 1 modulo $N$. Consider the finite group $\Delta=\mathcal{O}_{F}^{+, *} / U_{N}^{2}$. For $\epsilon \in \mathcal{O}_{F}^{+, *}$, we have an action $[\epsilon]: \bar{M}\left(\mu_{N}, \mathfrak{c}\right) \rightarrow \bar{M}\left(\mu_{N}, \mathfrak{c}\right)$ given by multiplying the polarization $\lambda$ by $\epsilon$. The action factors through $\Delta$ (see [2, intro., p. 6]). 
Lemma 8.1 The group $\Delta$ acts freely on $\bar{M}\left(\mu_{N}, \mathfrak{c}\right)$. One can form the quotient $\bar{M}\left(\mu_{N}, \mathfrak{c}\right)_{G}:=$ $\bar{M}\left(\mu_{N}, \mathfrak{c}\right) / \Delta$. The quotient map $\bar{M}\left(\mu_{N}, \mathfrak{c}\right) \rightarrow \bar{M}\left(\mu_{N}, \mathfrak{c}\right)_{G}$ is finite étale with group $\Delta$.

Proof Since $\bar{M}\left(\mu_{N}, \mathfrak{c}\right)$ is a projective scheme, it can be covered by open affine subschemes fixed by the action of $\Delta$. Thus we can form the quotient $\bar{M}\left(\mu_{N}, \mathfrak{c}\right)_{G}$. We now show that $\Delta$ acts freely on $\bar{M}\left(\mu_{N}, \mathfrak{c}\right)$. This can be proved over an algebraically closed field $k$ where the freeness of the action amounts to proving the following:

Consider an abelian variety with real multiplication $(A, \iota, \Psi, \lambda)$ over $k$ as in Sect. 3 and a totally positive unit $\epsilon \in \mathcal{O}_{F}^{+, *}$. Let $\alpha: A \rightarrow A$ be an automorphism commuting with the $\mathcal{O}_{F}$-action, the level $N$ structure $\Psi$ and such that $\lambda \circ \alpha=\epsilon \alpha^{\vee} \circ \lambda$. Then $\alpha \in U_{N}$ is a totally positive unit, congruent to 1 modulo $N$.

As $\alpha$ respects the level $N$ structure, it suffices to prove that $\alpha$ is an endomorphism lying in $\mathcal{O}_{F}$. Suppose this is not the case. Then $E:=F[\alpha] \subset \operatorname{End}^{0}(A)$ would be a commutative algebra of dimension at least $2 g$. It must be a field, else $A$ would decompose as a product of at least two abelian varieties of dimension less than $g$, with real multiplication by $F$ which is impossible. As a maximal commutative subalgebra of $\operatorname{End}^{0}(A)$ has dimension $\leq 2 g$, it follows that $E$ is a CM field of degree 2 over $F$. Moreover, the Rosati involution associated with any $\mathcal{O}_{F}$-invariant polarization induces complex conjugation on $E$. As the rank of the group of units in $\mathcal{O}_{E}$ is equal to the rank of the group of units of $\mathcal{O}_{F}$ by Dirichlet's unit theorem, it follows that there exists an integer $n \geq 2$ such that $\alpha^{n} \in \mathcal{O}_{F}^{*}$ and $\alpha^{n-1} \notin \mathcal{O}_{F}^{*}$. Hence $\zeta=(\alpha / \bar{\alpha})$ is a primitive $n$-root of unity in $\mathcal{O}_{E}$. It preserves every $\mathcal{O}_{F}$-equivariant polarization $\lambda$ as $\lambda^{-1} \circ \zeta^{\vee} \circ \lambda \circ \zeta=\bar{\zeta} \zeta=1$. As $N \geq 4$ it follows from Serre's lemma that $\zeta=1$ leading to a contradiction. We are left to show that $\Delta$ acts freely on the boundary $D:=\bar{M}\left(\mu_{N}, \mathfrak{c}\right)_{G} \backslash M\left(\mu_{N}, \mathfrak{c}\right)_{G}$. Recall that the boundary is the union of its connected components parametrized by the cusps of the minimal compactification. Each connected component of the boundary is stratified. More precisely, for each connected component, there is a polyhedral decomposition $\Sigma$ of the cone of totally positive elements $M^{+}$inside a fractional ideal $M \subset F$ determined by the cusp. To every cone $\sigma \in \Sigma$ corresponds a stratum $S_{\sigma} \subset D$. By construction of the toroidal compactification, if $\epsilon \in U_{N}$, then $S_{\epsilon^{2} \sigma}=S_{\sigma}$. We now claim that the action of $\Delta$ on the set of all strata in $D$ is free. This follows from the fact that the stabilizer of $\sigma \in \Sigma$ is a finite subgroup of $\mathcal{O}_{F}^{+, *}$ and thus trivial. This concludes the proof.

\subsection{Descending the sheaf $\mathfrak{w}^{\kappa^{\text {un }}}$}

We follow closely [2], see especially Sects. 1 and 4. First of all, the weight space associated with $G$ is the formal scheme $\mathfrak{W}_{F}^{G}$ defined by the Iwasawa algebra $\Lambda_{F}^{G}:=\mathbb{Z}_{p} \llbracket\left(\mathcal{O}_{F} \otimes\right.$ $\left.\mathbb{Z}_{p}\right)^{*} \times \mathbb{Z}_{p}^{*} \rrbracket$. There is a natural map of formal schemes $\mathfrak{W}_{F}^{G} \rightarrow \mathfrak{W}_{F}$ defined by the group homomorphism $\left(\mathcal{O}_{F} \otimes \mathbb{Z}_{p}\right)^{*} \rightarrow\left(\mathcal{O}_{F} \otimes \mathbb{Z}_{p}\right)^{*} \times \mathbb{Z}_{p}^{*}$ given by $t \mapsto\left(t^{2}, \operatorname{Nm}_{F / \mathbb{Q}}(t)\right)$. This induces a map of analytic adic spaces $\iota: \mathcal{W}_{F}^{G} \rightarrow \mathcal{W}_{F}$. We denote by $\kappa_{G}^{\text {un }}:=(v, w):\left(\mathcal{O}_{F} \otimes\right.$ $\left.\mathbb{Z}_{p}\right)^{*} \times \mathbb{Z}_{p}^{*} \rightarrow \Lambda_{F}^{G, *}$ the universal character.

Consider the formal scheme $\mathfrak{M}_{r} \times_{\mathfrak{W}_{F}} \mathfrak{W}_{F}^{G}$. Let $\mathfrak{w}_{G}^{\kappa_{G}^{\text {un }}}$ be the pullback of the universal sheaf to $\mathfrak{M}_{r} \times_{\mathfrak{W}_{F}} \mathfrak{W}_{F}^{G}$.

As a consequence of Lemma 8.1, the group $\Delta$ acts freely on the formal schemes $\mathfrak{M}_{r} \times_{\mathfrak{W}_{F}}$ $\mathfrak{W}_{F}^{G}$. We denote by $\mathfrak{M}_{r, G}$ the quotients by the group $\Delta$. We now claim that the action of $\Delta$ on $\mathfrak{M}_{r} \times_{\mathfrak{W}_{F}} \mathfrak{W}_{F}^{G}$ can be lifted to an action on the sheaf $\mathfrak{w}^{\kappa_{G}^{\text {un }}}$. 
Since $\mathfrak{w}^{\kappa_{G}^{\text {un }}}$ is defined without any reference to polarization, we have an isomorphism $\mu_{\epsilon}:[\epsilon]^{*} \mathfrak{w}^{\kappa_{G}^{\text {un }}} \rightarrow \mathfrak{w}^{\kappa_{G}^{\text {un }}}$ for all $\epsilon \in \mathcal{O}_{F}^{+, *}$. We modify the action by multiplying $\mu_{\epsilon}$ by $v(\epsilon)$. One then verifies, see $[2, \$ 4.1]$, that this action factors through the group $\Delta$. By finite étale descent, we obtain a sheaf that we continue to denote $\mathfrak{w}^{\kappa}{ }_{G}^{\text {un }}$ over $\mathfrak{M}_{r, G}$. We let $\mathcal{M}_{r, G}$ be the analytic fiber of $\mathfrak{M}_{r, G}$ and denote by $\omega^{\kappa_{G}^{\text {un }}}$ the invertible sheaf on $\mathcal{M}_{r, G}$ associated with $\mathfrak{w}^{\kappa_{G}^{\text {un }}}$.

\subsection{The cohomology of the sheaf $\omega_{G}^{\kappa_{G}^{\text {un }}}(-D)$}

There is an obvious map $\mathfrak{M}_{r, G} \rightarrow \overline{\mathfrak{M}}\left(\mu_{N}, \mathfrak{c}\right)_{G}$. Let $D$ denote the boundary divisor in $\overline{\mathfrak{M}}\left(\mu_{N}, \mathfrak{c}\right)_{G}$. We also denote by $D$ its inverse image in $\mathfrak{M}_{r, G}$.

Recall that $M^{*}\left(\mu_{N}, \mathfrak{c}\right)$ is the minimal compactification of $M\left(\mu_{N}, \mathfrak{c}\right)$. Certainly, the construction of $\mathfrak{M}_{r}$ admits a variant where one uses $M^{*}\left(\mu_{N}, \mathfrak{c}\right)$ as a starting point instead of $\bar{M}\left(\mu_{N}, \mathfrak{c}\right)$. Let us denote by $\mathfrak{M}_{r}^{\star}$ the resulting formal schemes.

We also define

$$
\mathfrak{M}_{r, G}^{\star}:=\left(\mathfrak{M}_{r}^{\star} \times \mathfrak{W}_{F} \mathfrak{W}_{F}^{G}\right) / \Delta
$$

Let $h: \mathfrak{M}_{r, G} \rightarrow \mathfrak{M}_{r, G}^{*}$ be the canonical projection. The main result of this section is the following cohomology vanishing theorem:

Theorem 8.2 We have $\mathrm{R}^{i} h_{\star} \mathfrak{w}^{\kappa_{G}^{\text {un }}}(-D)=0$ for all $i>0$.

Proof This is a variant of [2, Theorem 3.17]. Recall that $\bar{M}\left(\mu_{N}, \mathfrak{c}\right)_{G}$ is the quotient of $\bar{M}\left(\mu_{N}, \mathfrak{c}\right)$ by $\Delta$. Let $M^{*}\left(\mu_{N}, \mathfrak{c}\right)$ be the minimal compactification and let $M^{*}\left(\mu_{N}, \mathfrak{c}\right)_{G}$ be its quotient by $\Delta$. We have a map $h^{\prime}: \bar{M}\left(\mu_{N}, \mathfrak{c}\right)_{G} \rightarrow M^{*}\left(\mu_{N}, \mathfrak{c}\right)_{G}$. Let $\mathcal{L}$ be a torsion invertible sheaf on $\bar{M}\left(\mu_{N}, \mathfrak{c}\right)_{G}$. Then we claim that $\mathrm{R}^{i} h_{\star}^{\prime} \mathcal{L}(-D)=0$ for $i>0$. This follows from [2, Prop. 6.4]. Note that in that reference the proposition is stated for the trivial sheaf, but the proof works without any change for a torsion sheaf.

The map $h: \mathfrak{M}_{r, G} \rightarrow \mathfrak{M}_{r, G}^{*}$ is an isomorphism away from the cusps and in particular away from the ordinary locus, so we are left to prove the statement for the map $h_{\text {ord }}: \mathfrak{M}_{\text {ord,G }} \rightarrow \mathfrak{M}_{\text {ord,G }}^{*}$ over the ordinary locus. In this case, the sheaf $\mathfrak{w}^{\kappa_{G}^{\text {un }}}(-D)$ is invertible. Recall that the ring $\Lambda_{F}^{G}$ is semi-local and complete. Let $\mathfrak{n}$ be a maximal ideal of $\Lambda_{F}^{G}$ corresponding to a character $\eta:\left(\mathcal{O}_{F} / \mathfrak{p} \mathcal{O}_{F}\right)^{*} \times\left(\mathbb{F}_{p}\right)^{\times} \rightarrow \mathbb{F}_{q}^{\times}$where $\mathbb{F}_{q}$ is a finite extension of $\mathbb{F}_{p}$. We are left to prove the vanishing for the sheaf $\mathcal{F}:=\mathfrak{w}^{\kappa_{G}^{\text {un }}}(-D) / \mathfrak{n}$ over

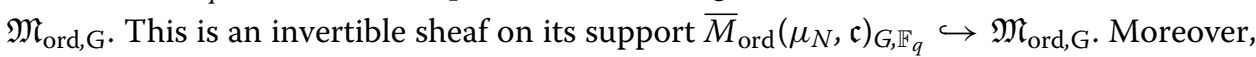
$\mathcal{F}^{\otimes q-1}=\mathcal{O}_{\bar{M}_{\text {ord }}\left(\mu_{N}, \mathfrak{c}\right)_{G, \mathbb{F} q}}$ because the order of the character $\eta$ divides $q-1$, and we can conclude.

Corollary 8.3 Let $g: \mathcal{M}_{r, G} \rightarrow \mathcal{W}_{F}^{G}$ be the projection to the weight space.

1. We have the following vanishing result: $\mathrm{R}^{i} g_{\star} \omega^{\kappa_{G}^{\mathrm{un}}}(-D)=0$ for all $i>0$.

2. For every point $\kappa \in \mathcal{W}_{G}$,

$$
\kappa^{*} g_{\star} \omega^{\kappa_{G}^{\mathrm{un}}}(-D)=\mathrm{H}^{0}\left(\mathcal{M}_{r, G}, \kappa^{\star} \omega^{\kappa_{G}^{\mathrm{un}}}(-D)\right)
$$

is the space of r-overconvergent, cuspidal, arithmetic Hilbert modular forms of weight $\kappa$.

3. There exists a finite covering of the weight space $\mathcal{W}_{F}^{G}=\cup_{i} \operatorname{Spa}\left(R_{i}, R_{i}^{+}\right)$such that

$$
g_{\star} \omega_{G}^{\kappa_{G}^{\text {un }}}(-D)\left(\operatorname{Spa}\left(R_{i}, R_{i}^{+}\right)\right)
$$

is a projective Banach $R_{i}$-module, for every $i$. 
Proof We have a sequence of maps

$$
g: \mathcal{M}_{r, G} \stackrel{g_{1}}{\rightarrow} \mathcal{M}_{r, G}^{*} \stackrel{g_{2}}{\rightarrow} \mathcal{W}_{F}^{G} .
$$

The map $g_{2}$ is affine and therefore has no nonzero higher cohomology. Moreover, Theorem 8.2 implies that $\mathrm{R}^{i}\left(g_{2}\right)_{\star} \omega^{\kappa_{G}^{\text {un }}}(-D)=0$. The second point follows easily.

For the last point, fix some open $\operatorname{Spa}\left(R_{i}, R_{i}^{+}\right) \subset \mathcal{W}_{F}^{G}$. Since the sheaf $\omega_{G}^{\kappa_{G}^{\text {un }}}(-D)$ is invertible, there is a finite covering $\cup \operatorname{Spa}\left(S_{i}, S_{j}^{+}\right)$of $\left.\mathcal{M}_{r, G}\right|_{\operatorname{Spa}\left(R_{i}, R_{i}^{+}\right)}$such that the sheaf $\omega^{\kappa_{G}^{\text {un }}}(-D)$ is trivial on every open of this covering. Arguing as in [3, Prop. 6.9], one proves that each $S_{i}$ is a projective Banach $R_{i}$-module. We can form the Chech complex associated with the covering $\cup \operatorname{Spa}\left(S_{i}, S_{j}^{+}\right)$, which, by (1), is a resolution of $g_{\star} \omega^{\kappa_{G}^{\mathrm{un}}}(-D)\left(\operatorname{Spa}\left(R_{i}, R_{i}^{+}\right)\right)$. Moreover, each term appearing in the complex is a projective Banach module and thus $g_{\star} \omega_{G}^{\kappa_{G}^{\text {un }}}(-D)\left(\operatorname{Spa}\left(R_{i}, R_{i}^{+}\right)\right)$is a projective Banach module.

\subsection{Koecher principle and $q$-expansions}

Consider the open moduli scheme $M\left(\mu_{N}\right)$ in place of $\bar{M}\left(\mu_{N}\right)$. Correspondingly we obtain an adic open subspace $\mathcal{M}_{r}^{o}$ of the adic space $\mathcal{M}_{r}$ introduced in Sect. 6.4. Let $g^{o}: \mathcal{M}_{r}^{o} \rightarrow \mathcal{W}_{F}$ and $g: \mathcal{M}_{r} \rightarrow \mathcal{W}_{F}$ be the structural maps. Similarly, in the arithmetic case we have an adic open subspace $\mathcal{M}_{r, G}^{o}$ of the adic space $\mathcal{M}_{r, G}$ and we denote again by $g^{o}$, resp. $g$ the structural morphisms to $\mathcal{W}_{F}^{G}$ (see Sect. 8.2). The Koecher principle states that:

Proposition 8.4 The natural morphisms $g_{*}^{o} \omega^{\kappa^{\mathrm{un}}} \rightarrow g_{*} \omega^{\kappa^{\mathrm{un}}}$ and $g_{*}^{o} \omega_{G}^{\kappa_{G}^{\mathrm{un}}} \rightarrow g_{*} \omega^{\kappa_{G}^{\mathrm{un}}}$ are isomorphisms.

Proof Since the complement of $M\left(\mu_{N}\right)$ in $\bar{M}\left(\mu_{N}\right)$ is contained in the ordinary locus, one may restrict to proving the two assertions on ordinary loci. This is classical: Unraveling the constructions one is reduced to prove the claims for the structural sheaves on the adic Igusa tower. This can be proven at the level of formal schemes and then, by devissage, reducing to the Igusa tower $\overline{I G}_{n}^{\text {ord }, o} \subset \overline{I G}_{n}^{\text {ord }}$ modulo $p$ over $\bar{M}\left(\mu_{N}\right)_{\mathbb{F}_{p}}^{\text {ord }}$.

Let $f_{n}: \overline{I G}_{n}^{\text {ord }} \rightarrow \bar{M}\left(\mu_{N}\right)_{\mathbb{F}_{p}}^{\text {ord }}$ be the structural map. Notice that $\bar{M}\left(\mu_{N},\right)_{\mathbb{F}_{p}}^{\text {ord }}$ is smooth, the minimal compactification $\bar{M}^{*}\left(\mu_{N}\right)_{\mathbb{F}_{p}}^{\text {ord }}$ is normal and the map $\gamma: \bar{M}\left(\mu_{N}\right)_{\mathbb{F}_{p}}^{\text {ord }} \rightarrow \bar{M}^{*}\left(\mu_{N}\right)_{\mathbb{F}_{p}}^{\text {ord }}$ is proper birational and it is an isomorphism outside a codimension 2 locus. Then

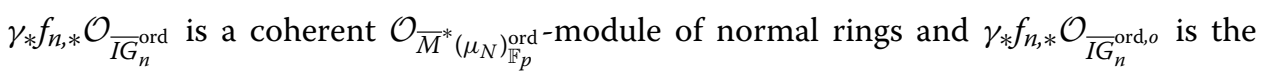
complement of a codimension two loci. Thus the map on global sections $\gamma_{*} f_{n, *} \mathcal{O}_{\overline{I G}_{n, r, \alpha, I}^{\text {ord }}} \rightarrow$ $\gamma_{*} f_{n, *} \mathcal{O}_{\overline{I G_{n, r, \alpha, I}}}^{\text {ord }, I}$ is an isomorphism concluding the proof of the Proposition.

The restriction of our construction to the ordinary locus gives back the theory of families of $p$-adic modular forms of Katz [14] and Hida [11]; see [4, \$5.4] for details. In particular, one gets $q$-expansions at the cusps.

\subsection{Hecke operators}

Consider a polarization module $\mathfrak{c}$ as in Sect. 3. In this section, we work with the open moduli scheme $M\left(\mu_{N}, \mathfrak{c}\right)$ in place of $\bar{M}\left(\mu_{N}, \mathfrak{c}\right)$ in order to avoid the problem of finding toroidal compactifications preserved by the Hecke operators and we will use the Koecher principle to extend these operators over the cusps. Corresponding to the open subscheme $M\left(\mu_{N}, \mathfrak{c}\right)$ of $\bar{M}\left(\mu_{N}, \mathfrak{c}\right)$, we get an open formal scheme $\mathfrak{X}_{r}^{o}(\mathfrak{c})$ of $\mathfrak{X}_{r}(\mathfrak{c})$, proceeding as in Sect. 3.4. 
Let $\ell$ be an ideal of $\mathcal{O}_{F}$ prime to $N$. If $\ell$ is not prime to $p$, we assume that it is either equal to $p \mathcal{O}_{F}$ or that it is prime. Proceeding as in [2, \$3.7], we consider the normalization $\mathfrak{S}_{\ell, r} \subset \mathfrak{X}_{r}^{o}(\mathfrak{c}) \times \mathfrak{X}_{r}^{o}(\ell \mathfrak{c})$ of the Hecke correspondence classifying isogenies $\pi_{\ell}: G \rightarrow G^{\prime}$, where $G$ and $G^{\prime}$ are defined by points of $\mathfrak{X}_{r}^{o}(\mathfrak{c})$ and $\mathfrak{X}_{r}^{o}(\ell \mathfrak{c})$ respectively and $\pi_{\ell}$ is an isogeny of degree $\left|\mathcal{O}_{F} / \ell \mathcal{O}_{F}\right|$ compatible with $\mathcal{O}_{F}$-actions and polarizations, Ker $\pi_{\ell} \subset G[\ell]$ and such that (1) Ker $\pi_{\ell}$ is étale locally isomorphic to $\mathcal{O}_{F} / \ell \mathcal{O}_{F}$, if $\ell$ does not divide $p$ or (2) the scheme theoretic intersection of $\operatorname{Ker} \pi_{\ell}$ with the canonical subgroup $H_{1}$ of $G$ is trivial, if $\ell$ is prime and divides $p$ or (3) the morphism $\operatorname{Ker} \pi_{\ell} \rightarrow G[p] / H_{1}$ is an isomorphism, if $\ell=p \mathcal{O}_{F}$.

We have the two natural projections $p_{1}: \mathfrak{S}_{\ell, r} \rightarrow \mathfrak{X}_{r}^{o}(\mathfrak{c})$ and $p_{2}: \mathfrak{S}_{\ell, r} \rightarrow \mathfrak{X}_{r}^{o}(\ell \mathfrak{c})$.

Lemma 8.5 The universal isogeny $\pi_{\ell}$ induces an isomorphism $\pi_{\ell}^{*}: p_{1}^{*} \mathfrak{w}_{I} \rightarrow p_{2}^{*} \mathfrak{w}_{I}$, of the pullbacks via $p_{1}$ and $p_{2}$, respectively, of the invertible sheaves $\mathfrak{w}_{I}$ on $\mathfrak{X}_{r}^{o}(\mathfrak{c})$ and $\mathfrak{X}_{r}^{o}(\ell \mathfrak{c})$, respectively, defined in Theorem 6.4 .

Proof Over $\mathcal{W}_{F,[0,1]}$ this is the content of [2, Cor. 3.25] using the compatibility given in Theorem 6.7.

We deal with the general case. It follows from [3, Rmk A.1] and our assumptions on $\pi_{\ell}$ that the isogeny $\pi_{\ell}: G \rightarrow G^{\prime}$ induces an isomorphism from the canonical subgroup of level $n$ of $G$ to the canonical subgroup of level $n$ of $G^{\prime}$. This implies that $\pi_{\ell}$ defines an isomorphism of the pullback to $\mathfrak{S}_{\ell, r}$ via $p_{1}$ and $p_{2}$ of the Igusa towers. By the functoriality of the Hodge-Tate map we also get an isomorphism $p_{2}^{*} \mathcal{F} \rightarrow p_{1}^{*} \mathcal{F}$ of the modified integral structures $\mathcal{F}$ of $\omega_{G^{\prime}}$ and $\omega_{G}$ of Proposition 4.1 . Thus we get an isomorphism $p_{2}^{*} \mathfrak{F}_{n, r, I} \rightarrow$ $p_{1}^{*} \mathfrak{F}_{n, r, I}$ of the pullback to $\mathfrak{S}_{\ell, r}$ of the torsors $\mathfrak{F}_{n, r, I}$ of Sect. 4.1. Due to the normality of $\mathfrak{S}_{\ell, r}$, we get an isomorphism of the pull backs of the sheaves $p_{1}^{*} \mathfrak{w}_{n, r, I} \rightarrow p_{2}^{*} \mathfrak{w}_{n, r, I}$ of overconvergent forms defined in Proposition 4.3.

Similarly, $\pi_{\ell}$ defines an isomorphism between the pull backs of $\mathfrak{I} \mathfrak{G}_{\infty, \infty, I}$ via $p_{1}$ and $p_{2}$ respectively (see Sect. 5.2 for the definition). The analog of Lemma 5.4 over the base $\mathfrak{S}_{\ell, r}$ provides an isomorphism between the pullbacks via $p_{1}$ and $p_{2}$, respectively, of the sheaves $\mathfrak{w}_{I}^{\text {perf }}$ of perfect overconvergent forms defined in Proposition 5.5. Thanks to the normality of $\mathfrak{S}_{\ell, r}$ we deduce the sought for isomorphism of the pullbacks via $p_{1}$ and $p_{2}$ of the sheaves $\mathfrak{w}_{I}$.

Using the conventions of Sect. 6.3, we also have the normalization of the Hecke correspondences $p_{1}: \mathfrak{T}_{\ell, r} \rightarrow \mathfrak{Z}_{r}^{o}(\mathfrak{c})$ and $p_{2}: \mathfrak{S}_{\ell, r} \rightarrow \mathfrak{Z}_{r}^{o}(\ell \mathfrak{c})$ defined over the Iwasawa algebra. As above we denote by $\mathfrak{Z}_{r}^{o}(\mathfrak{c})$ the open formal subscheme of $\mathfrak{Z}_{r}(\mathfrak{c})$ associated with $M\left(\mu_{N}, \mathfrak{c}\right)$. Following the notations of Sect. 6.4, we let $\mathcal{M}_{r}^{o}(\mathfrak{c})$ be the adic analytic fiber of $\mathfrak{Z}_{r}^{o}(\mathfrak{c}) \times_{\mathfrak{W}_{F}^{0}} \mathfrak{W}_{F}$.

Corollary 8.6 The map $\pi_{\ell}$ induces an isomorphism

$$
\pi_{\ell}^{*}: p_{1}^{*} \omega^{\kappa^{\mathrm{un}}} \rightarrow p_{2}^{*} \omega^{\kappa^{\mathrm{un}}}
$$

of the sheaves $\omega^{\kappa^{\text {un }}}$ defined over $\mathcal{M}_{r}^{o}(\mathfrak{c})$ and $\mathcal{M}_{r}^{o}(\ell \mathfrak{c})$ respectively.

Proof We have a morphism $g: \mathfrak{S}_{\ell, r} \rightarrow \mathfrak{T}_{\ell, r}$ compatible with the natural projections $\mathfrak{Z}_{r}^{o}(\mathfrak{c}) \rightarrow \mathfrak{X}_{r}^{o}(\mathfrak{c})$ and $\mathfrak{X}_{r}^{o}(\mathfrak{c}) \rightarrow \mathfrak{Z}_{r}^{o}(\mathfrak{c})$. As in Theorem 6.6(1) and using the normality of $\mathfrak{T}_{\ell, r}$, one obtains that the natural morphism $\mathcal{O}_{\mathfrak{T}_{\ell, r}} \rightarrow g_{*} \mathcal{O}_{\mathfrak{S}_{\ell, r}}$ is an isomorphism. This implies that the isomorphism of Lemma 8.5 induces an isomorphism of the pull backs via $p_{1}$ and $p_{2}$, respectively, of the invertible sheaves $\mathfrak{w}^{\kappa}$ defined on $\mathfrak{Z}_{r}^{o}(\mathfrak{c})$ and $\mathfrak{Z}_{r}^{o}(\ell \mathfrak{c})$, respectively. 
Taking adic analytic fibers and twisting by the invertible sheaf $\mathfrak{w}^{\chi}$ as in Sect. 6.4 we get the claimed isomorphism.

Let $q_{\ell}:=\left|\mathcal{O}_{F} / \ell \mathcal{O}_{F}\right|$. In order to define the Hecke operators, we will need the following:

Lemma 8.7 (1) The morphism $p_{1}$ is finite and étale of degree $q_{\ell}+1$ if $\ell$ is prime to $p$.

(2) At the level of analytic fibers, the morphism $p_{1}$ is finite and flat of degree $q_{\ell}$ if $\ell$ divides $p$.

Proof The first statement is clear. We prove the second statement: The group scheme $\operatorname{Ker} \pi_{\ell} \cong G[\ell] / H_{1}[\ell]$ is finite étale at the level of analytic fibers due to [3, Cor. A.2]. Since it is isomorphic to $H_{1}[\ell]^{\vee}$ it follows from Proposition 3.2 that it is in fact étale locally on $\mathcal{M}_{r}^{o}(\mathfrak{c})$ isomorphic to $\mathcal{O}_{F} / \ell \mathcal{O}_{F}$. Since $p_{1}$ is represented over $\mathcal{M}_{r}^{o}(\mathfrak{c})$ by the $\mathcal{O}_{F}$ splittings of the exact sequence of group schemes (with $\mathcal{O}_{F}$-action) $0 \rightarrow H_{1}[\ell] \rightarrow G[\ell] \rightarrow$ $G[\ell] / H_{1}[\ell] \rightarrow 0$, it follows that $p_{1}$ is a torsor under the finite and flat group scheme $H_{1}[\ell]$. This proves the claim.

Denote by $g$ the structural maps of $\mathcal{M}_{r}(\ell \mathfrak{c})$ and respectively $\mathcal{M}_{r}(\mathfrak{c})$ to the weight space $\mathcal{W}_{F}$ and by $g^{o}$ their restrictions to $\mathcal{M}_{r}^{o}(\ell \mathfrak{c})$ and respectively $\mathcal{M}_{r}^{o}(\mathfrak{c})$. Taking the global sections of the sheaves $\omega^{\kappa}$ un over $\mathcal{M}_{r}^{o}(\ell \mathfrak{c})$ and respectively $\mathcal{M}_{r}^{o}(\mathfrak{c})$, we get the morphism

$$
g_{*}^{o} \omega^{\kappa^{\mathrm{un}}} \rightarrow g_{*}^{o}\left(p_{1, *}\left(p_{2}^{*} \omega^{\kappa^{\mathrm{un}}}\right)\right) \stackrel{\pi_{\ell}^{*,-1}}{\longrightarrow} g_{*}^{o}\left(p_{1, *}\left(p_{1}^{*} \omega^{\kappa^{\mathrm{un}}}\right)\right)^{q_{\ell}^{-1} \operatorname{Tr}_{p_{1}}} g_{*}^{o} \omega^{\kappa^{\mathrm{un}}}
$$

Here $\operatorname{Tr}_{p_{1}}$ is the trace map of the finite flat morphism $p_{1}$. One checks on $q$-expansions that dividing by the normalization factor $q_{\ell}$ is a well-defined operation. Using the Koecher principle of Sect. 8.4, we get a map $g_{*} \omega^{\kappa^{\text {un }}} \rightarrow g_{*} \omega^{\kappa^{\text {un }}}$ from the global sections of $\omega^{\kappa^{\text {un }}}$ over $\mathcal{M}_{r}(\ell \mathfrak{c})$ to the global sections over $\mathcal{M}_{r}(\mathfrak{c})$, which are denoted $T_{\ell}$, for $\ell$ not dividing $p$, and $U_{\ell}$, for $\ell=p \mathcal{O}_{F}$ or a prime ideal dividing $p$. This provides the definition of the Hecke operators for the overconvergent (cuspidal) forms defined in Theorem 6.7 for the group $G^{*}$. Taking the quotient under the group $\Delta$, we define such Hecke operators also for the arithmetic overconvergent cuspidal modular forms defined in Sect. 8.2 for the group $G$.

\subsection{The adic eigenvariety for arithmetic Hilbert modular forms}

In [2, Theorem 5.1], we constructed a cuspidal eigenvariety over $\mathcal{W}_{F}^{G} \backslash\{|p|=0\}$ for the $p$-adic, arithmetic Hilbert modular forms. We now extend it over $\mathcal{W}_{F}^{G}$.

Let $\operatorname{Frac}(F)^{(p)}$ be the group of fractional ideals prime to $p$. Let $\operatorname{Princ}(F)^{+,(p)}$ be the group of positive elements which are $p$-adic units. The quotient $\operatorname{Frac}(F)^{(p)} / \operatorname{Princ}(F)^{+,(p)}=\mathrm{Cl}^{+}(F)$ is the strict class group of $F$.

For all $\mathfrak{c} \in \operatorname{Frac}(F)^{(p)}$, we have defined an adic space $\mathcal{M}_{r}$ that we now denote by $\mathcal{M}_{r}(\mathfrak{c})$ in order to mark its dependance on $\mathfrak{c}$ and a sheaf $\omega^{\kappa_{G}^{\text {un }}}$ over $\mathcal{M}_{r}(\mathfrak{c})$. Let $g_{\mathfrak{c}}: \mathcal{M}_{r}(\mathfrak{c}) \rightarrow \mathcal{W}_{F}^{G}$ be the projection. For all $x \in \operatorname{Princ}(F)^{+,(p)}$, we canonically identify $\left(g_{\mathfrak{c}}\right)_{\star} \omega^{\kappa_{G}^{\text {un }}}(-D)$ and $\left(g_{x \mathfrak{c}}\right)_{\star} \omega^{\kappa_{G}^{\text {un }}}(-D)$ as in [2, Def. 4.6].

Taking the limit over $r$, we thus obtain a sheaf of projective Banach modules

$$
\bigoplus_{\mathfrak{c} \in \mathrm{Cl}^{+}(F)}\left(g_{\mathfrak{c}}\right)_{\star} \omega^{\kappa_{G}^{\text {un }}}(-D) \text {. }
$$

Thanks to Sect. 8.5 this sheaf carries an action of the Hecke algebra $\mathcal{H}^{p}$ of level prime to $p$ as well as an action of the $U_{p}$ operator and of the operators $U_{\mathfrak{P}_{i}}$ (see $\left.[2, \mathbb{\$} 4.3]\right)$. Moreover, having taken the limit over $r$ implies the $U_{p}$-operator is compact thanks to Proposition 3.3. Applying [3, Appendice B], we obtain the following theorem. 
Theorem 8.8 $\quad$ 1. The characteristic series

$$
P^{G}(X)=\operatorname{det}\left(1-X U_{p} \mid \bigoplus_{\mathfrak{c} \in \mathrm{Cl}^{+}(F)}\left(g_{\mathfrak{c}}\right)_{\star} \omega^{\kappa_{G}^{\mathrm{un}}}(-D)\right)
$$

takes values in $\Lambda_{F}^{G} \llbracket X \rrbracket$.

2. The spectral variety $\mathcal{Z}^{G}=V\left(P^{G}\right) \rightarrow \mathcal{W}_{F}^{G}$ is locally finite, flat and partially proper over the weight space.

3. There is an eigenvariety $\mathcal{E}^{G} \rightarrow \mathcal{Z}^{G}$, finite and torsion free over $\mathcal{Z}^{G}$, which parametrizes finite slope eigensystems of overconvergent, arithmetic Hilbert modular forms.

\section{Author details}

${ }^{1}$ Concordia University, Montreal, Canada, ${ }^{2}$ Università degli Studi di Padova, Padova, Italy, ${ }^{3}$ Università Statale di Milano,

Milano, Italy, ${ }^{4}$ École Normale Supérieure de Lyon, Lyon, France.

\section{Acknowledgements}

We thank the referee for the careful reading of the manuscript and his/her remarks which hopefully led to improvements The Remark 1.1 was written at his/her request. During part of the work on this project, Vincent Pilloni was supported by ANR PerCoLaTor.

During part of the work on this article Fabrizio Andreatta was supported by the Italian grant Prin 2010/2011.

\section{Authors' contributions}

We dedicate this article to the memory of Robert Coleman, whose ideas and insights inspired it.

Received: 31 July 2015 Accepted: 25 May 2016

Published online: 08 September 2016

\section{References}

1. Andreatta, F., Goren, E.Z.: Hilbert modular forms: mod $p$ and p-adic aspects. AMS Mem. 173(819), (2005) (vi+100)

2. Andreatta, F., lovita, A., Pilloni, V.: On overconvergent Hilbert cusp forms. Asterisque (2014) (preprint)

3. Andreatta, F., lovita, A., Pilloni, V.: Le halo spectrale (2015) (preprint)

4. Andreatta, F., lovita, A., Pilloni, V.: p-adic families of Siegel modular cuspform. Ann. Math. 181, 623-697 (2015)

5. Andreatta, F., lovita, A., Stevens, G.: Overconvergent modular sheaves and modular forms for $\mathbf{G L}(2, F)$. Isr. J. Math. 201, 299-359 (2014)

6. Coleman, R.: Classical and overconvergent modular forms. Invent. Math. 124, 215-241 (1996)

7. Coleman, R.: p-adic Banach spaces and families of modular forms. Invent. Math. 127, 417-479 (1997)

8. Coleman, R., Mazur, B.: The eigencurve. In: Galois Representations in Arithmetic Algebraic Geometry (Durham, 1996), London Mathematical Society. Lecture Note Series, vol. 254, pp. 1-113. Cambridge University Press, Cambridge (1998)

9. Deligne, P., Pappas, G.: Singularités des espaces des modules de Hilbert, en les Caractéristiques divisant le discriminant. Compos. Math. 90, 59-79 (1994)

10. Hida, H.: Iwasawa modules attached to congruences of cusp forms. Ann. Sci. École Norm. Supér. 19, $231-273$ (1986)

11. Hida, H.: Control theorems for coherent sheaves on Shimura varieties of PEL type. J. Inst. Math. Jussieu 1, 1-76 (2002)

12. Huber, R.: Continuous valuations. Math. Z. 212, 455-477 (1993)

13. Huber, R.: A generalization of formal scheme and rigid analytic varieties. Math. Z. 217, 513-551 (1994)

14. Katz, N.: P-adic properties of modular schemes and modular forms. In: Kuijk, W., Serre, J.-P. (eds.) Modular Functions of One Variable III. Lecture Notes in Mathematics, vol. 350, pp. 69-190. Springer, Berlin

15. Pappas, G., Rapoport, M.: Local models in the ramified case, II: splitting models. Duke Math. J. 127, 193-250 (2005)

16. Rapoport, M.: Compactifications de l'espace de modules de Hilbert-Blumenthal. Compos. Math. 36, $255-335$ (1978)

\section{Submit your manuscript to a SpringerOpen ${ }^{\circ}$ journal and benefit from:}

- Convenient online submission

Rigorous peer review

- Immediate publication on acceptance

Open access: articles freely available online

- High visibility within the field

- Retaining the copyright to your article

Submit your next manuscript at springeropen.com 